\title{
Self-Interacting Fermionic Dark Matter with a Scalar Mediator \\ by
}

\author{
William Scott
}

A thesis submitted to the Faculty of Graduate and Postdoctoral Affairs in partial fulfilment of the requirements for the degree of

\author{
Master of Science \\ in \\ Physics \\ Ottawa-Carleton Institute for Physics \\ Carleton University \\ Ottawa, Ontario
}

(C)2016, William Scott 


\begin{abstract}
Recent observations of galaxy cluster Abell 3827 reveal a spatial offset between the galaxies and their associated dark matter halos [1]. It has been suggested that dark matter self-interactions of strength $1.0 \mathrm{~cm}^{2} / \mathrm{g} \lesssim \sigma / \mathrm{m} \lesssim$ $1.5 \mathrm{~cm}^{2} / \mathrm{g}$ could be responsible for the observed discrepancies [2]. We propose a simple model for the dark sector consisting of a Dirac fermion and a scalar mediator. This dark sector is allowed to communicate with the Standard Model through mixing of the scalar mediator with the Higgs. Examination of the dark matter's thermal freeze-out from p-wave annihilation, in combination with a theoretical upper bound placed on the mediator-fermion coupling strength, indicates that in order to satisfy the observations of Abell 3827, the mediator must be extremely light - between $0.69 \mathrm{MeV}$ and $100 \mathrm{MeV}$ - whereas the dark matter fermion could have a mass from $0.35 \mathrm{GeV}$ to $8200 \mathrm{GeV}$. Analysis of the Higgs-mediator mixing reveals an allowed range of mediator lifetimes, from $3.2 \times 10^{-10} \mathrm{~s}$ to $10^{-2} \mathrm{~s}$.
\end{abstract}




\section{Acknowledgements}

I would like to thank several people for their aid during my time spent conducting research at Carleton University.

First, I would like to thank those who did their research alongside meAlex, Robyn, Hassan and Ben-for their friendship and helpful suggestions. In particular, I would like to thank Gage Bonner, whose computational brilliance allowed him to find much more effective methods of performing freeze-out calculations than I did, and who therefore saved me from months of gruelling simulations. I would also like to thank Gage for introducing me to software that is capable of solving difficult integrals quickly. I believe he summed up his helpfulness well when, with incredulity, he stated, "I don't know how you made it this far in your physics career without this stuff."

I would also like to thank my friends and family for any and all support they have provided.

Finally, I am most thankful to my supervisor, Dr. Heather Logan, whose patience knows no bounds and who was always willing to explain things a second (or third) time. Her guidance has been absolutely invaluable, and I consider myself very lucky to have had her for a supervisor. 


\section{Statement of Originality}

All of the content presented in this thesis is the original work of the author. The image showing an example of gravitational lensing in Fig. 2.4 was taken from NASA, and its use is permitted under NASA's media usage guidelines. Fitting functions used to generate Fig. 4.1 and Fig. 4.3 were provided by Gage Bonner and were useful in furthering arguments provided in Ch. 4. This thesis makes predictions about the nature of a particular dark matter model when incorporating the effects of self-interactions as outlined in Ref. [1], which, to the best of our knowledge, has not been done before. 


\section{Contents}

1 Introduction 1

2 Dark Matter 5

2.1 Evidence.................... 6

2.2 Composition . . . . . . . . . . . . . . . . . . . . . . . . 12

2.3 Self-Interactions . . . . . . . . . . . . . . 16

3 A Candidate Model $\quad 20$

3.1 Particles and Feynman Rules . . . . . . . . . . . . . . . 21

3.2 Amplitudes . . . . . . . . . . . . . . . . . . . . 27

3.3 Scattering in the Non-Relativistic Limit . . . . . . . . . . . . . 41

4 Thermal Relics $\quad 46$

4.1 Freeze-Out..................... 47

4.2 Deriving $\left\langle\sigma_{\text {ann }} v_{\text {rel }}\right\rangle \ldots \ldots \ldots \ldots$. . . . . . . . . . . . 51

4.3 Determining $\alpha \ldots \ldots \ldots$. . . . . . . . . . . 61

4.4 A Relationship Between $m_{\chi}$ and $m_{\phi}$. . . . . . . . . . . 65 
5 The Dark Sector Meets the Standard Model

5.1 Mixing the $\phi$ with the Higgs . . . . . . . . . . . . . . 76

5.2 Lifetimes for the $\tilde{\phi} \ldots \ldots$. . . . . . . . . . . . 83

6 Conclusions $\quad 90$

$\begin{array}{ll}\text { Appendices } & 92\end{array}$

$\begin{array}{ll}\text { A Units } & 92\end{array}$

B Application of the Runge-Kutta Method to Freeze-Out 95

C The Scattering Cross Section Integrals 100 


\section{List of Tables}

1 Relevant Constants ................. ix

2 Particle Information ................ . . $\mathrm{x}$

A.1 Unit Conversions . . . . . . . . . . . . . . . . . . 94 


\section{List of Figures}

2.1 A Redshifted Absorption Spectrum . . . . . . . . . . . 8

2.2 Variables Needed to Calculate Rotation Curves . . . . . . . . 10

2.3 A Typical Rotation Curve . . . . . . . . . . . . . . . . . . 11

2.4 An Example of Gravitational Lensing . . . . . . . . . . . . . . 11

2.5 Mass Distribution in Abell 3827 Schematic . . . . . . . . . . 18

3.1 A Labelled Diagram Depicting a Scattering Event . . . . . . . 36

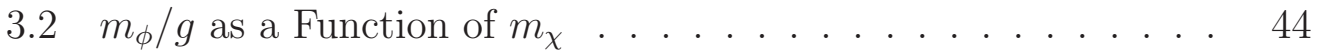

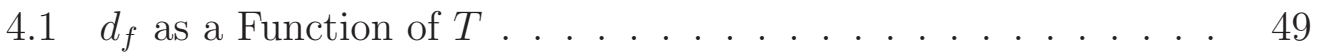

4.2 Freeze Out for $m_{\chi}=100 \mathrm{GeV}$. . . . . . . . . . . 63

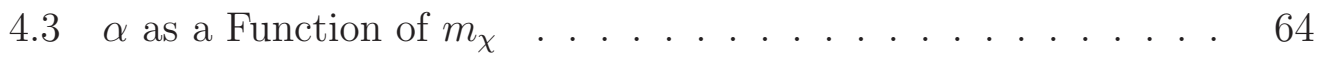

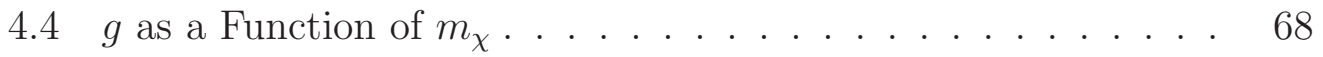

4.5 A Poorly Approximated $m_{\phi}$ vs $m_{\chi}$ Plot . . . . . . . . . . . . 69

4.6 An Improved $m_{\phi}$ vs $m_{\chi}$ Plot . . . . . . . . . . . . . . . . 74

$5.1 \tau$ as a Function of $m_{\tilde{\phi}} \ldots \ldots \ldots$. . . . . . . . . . . . . . 88 


\section{Table 1 - Relevant Constants}

\begin{tabular}{lll}
\hline \hline Description & Symbol & Value \\
\hline Fine structure constant & $\alpha_{\mathrm{F}}$ & $7.297 \times 10^{-3}$ \\
Gravitational constant & $G$ & $6.674 \times 10^{-11} \mathrm{~m}^{3} \mathrm{~kg}^{-1} \mathrm{~s}^{-2}$ \\
Reduced Planck's constant & $\hbar$ & $1.055 \times 10^{-34} \mathrm{~J} \mathrm{~s}^{-1}$ \\
Boltzmann's constant & $k_{\mathrm{B}}$ & $1.381 \times 10^{-23} \mathrm{~J} \mathrm{~K}^{-1}$ \\
Speed of light & $c$ & $2.998 \times 10^{8} \mathrm{~m} \mathrm{~s}^{-1}$ \\
Hubble expansion rate scale factor & $h$ & $0.678 \pm 0.009$ \\
Present Hubble expansion rate & $H_{0}$ & $100000 h \mathrm{~m} \mathrm{~s}^{-1} \mathrm{Mpc}^{-1}$ \\
Cold dark matter density parameter & $\Omega_{\mathrm{CDM}}$ & $(0.1186 \pm 0.0020) h^{-2}$ \\
Present CMB temperature & $T_{0}$ & $2.273 \mathrm{~K}$ \\
Fundamental unit of charge & $e$ & $1.602 \times 10^{-19} \mathrm{C}$ \\
\hline
\end{tabular}

Table 1: Relevant physical constants as given in Ref. [3]. Uncertainties are included for those quantities that are known to fewer than four significant figures. In all other cases, quantities are reported to four significant figures. The quantities are expressed in SI units $\left(1 \mathrm{Mpc}=3.0857 \times 10^{22} \mathrm{~m}\right)$. For a description of how to convert to natural units such that $\hbar=c=k_{\mathrm{B}}=1$, see App. A. 
Table 2 - Particle Information

\begin{tabular}{llllll}
\hline \hline Particle & Symbol & Mass & Charge & Spin & $N_{\mathrm{c}}$ \\
\hline Higgs boson & $h$ & $125.1 \mathrm{GeV}$ & 0 & 0 & 0 \\
$W$ boson & $W^{ \pm}$ & $80.39 \mathrm{GeV}$ & $\pm e$ & 1 & 0 \\
$Z$ boson & $Z^{0}$ & $91.19 \mathrm{GeV}$ & 0 & 1 & 0 \\
photon & $\gamma$ & 0 & 0 & 1 & 0 \\
gluon & $g$ & 0 & 0 & 1 & 8 \\
up quark & $u$ & $\approx 2.2 \mathrm{MeV}$ & $\frac{2}{3} e$ & $1 / 2$ & 3 \\
down quark & $d$ & $\approx 4.7 \mathrm{MeV}$ & $-\frac{1}{3} e$ & $1 / 2$ & 3 \\
strange quark & $s$ & $\approx 96 \mathrm{MeV}$ & $-\frac{1}{3} e$ & $1 / 2$ & 3 \\
charm quark & $c$ & $1.27 \pm 0.03 \mathrm{GeV}$ & $\frac{2}{3} e$ & $1 / 2$ & 3 \\
bottom quark & $b$ & $4.18 \pm 0.04 \mathrm{GeV}$ & $-\frac{1}{3} e$ & $1 / 2$ & 3 \\
top quark & $t$ & $173.21 \pm 0.71 \mathrm{GeV}$ & $\frac{2}{3} e$ & $1 / 2$ & 3 \\
electron & $e$ & $0.5110 \mathrm{MeV}$ & $-e$ & $1 / 2$ & 0 \\
muon & $\mu$ & $105.7 \mathrm{MeV}$ & $-e$ & $1 / 2$ & 0 \\
tau & $\tau$ & $1777 \mathrm{MeV}$ & $-e$ & $1 / 2$ & 0 \\
electron neutrino & $\nu_{e}$ & $<2 \mathrm{eV}$ & 0 & $1 / 2$ & 0 \\
muon neutrino & $\nu_{\mu}$ & $<2 \mathrm{eV}$ & 0 & $1 / 2$ & 0 \\
tau neutrino & $\nu_{\tau}$ & $<2 \mathrm{eV}$ & 0 & $1 / 2$ & 0 \\
\hline
\end{tabular}

Table 2: Mass, charge, spin and number of colours $\left(N_{\mathrm{c}}\right)$ of the fundamental particles of the Standard Model as given in Ref. [3]. All fermions have associated antiparticles. Where possible, nonzero masses are reported to four significant figures. The up, down and strange masses are notoriously imprecise; and the charm, bottom and top masses are given as accurately as possible. The neutrino masses are unknown. The masses are reported in natural units. To convert to SI units, see App. A. 


\section{Chapter 1}

\section{Introduction}

The universe appears to be full of some sort of non-baryonic matter that cannot be observed directly but that makes a very conspicuous gravitational imprint [4]. It is called dark matter, and though a few within the scientific community believe it might not exist and that our gravitational equations might be incorrect at certain scales, the general consensus is that dark matter is made up of particles. (For a pedagogical review, see Ref. [5].)

There are a number of competing theories attempting to describe the nature of dark matter particles [6]. One such theory is that axions, particles originally proposed to solve a mystery within quantum chromodynamics, might comprise dark matter. Most theories, however, postulate that dark matter is made up of particles that interact via the weak force or by some unknown force

of similar magnitude. Termed WIMPs (Weakly Interacting Massive Particles), these particles are thought likely to exist because of the so-called WIMP mir- 
acle, the fact that non-baryonic matter in the early universe would have had to interact with a strength on the order of the weak scale in order for the right amount of dark matter to exist today. (See, for example, Ref. [7].)

Recent observations of a particular galaxy cluster suggest dark matter interacts with itself more strongly than it does with Standard Model particles [1]. Given the limited evidence for the phenomenon, very few models incorporate dark matter self-interactions, but work has been done attempting to explain the galaxy cluster observations with a dark matter model involving only a single scalar particle [8]. However, that study demonstrated the need for significant fine-tuning, in particular because the scalar could not satisfy freeze-out requirements.

In an attempt to find a more agreeable explanation for the self-interaction observations, we propose a model for dark matter inspired by Ref. [9] that includes a matter-antimatter pair of spin-half fermions that interact with one another via the exchange of a scalar mediator. In this way, self-interactions can be on the order of the weak scale while allowing Standard Model particles to remain largely unaffected by the dark matter. The only way the dark matter can interact with the Standard Model is through mixing of the mediator and the Higgs boson. This mixing affects our model very little except that it forces the dark matter to remain in thermal equilibrium with Standard Model particles during the first few moments after the Big Bang.

This thesis is divided into four major chapters (Ch. 2, Ch. 3, Ch. 4 and 
Ch. 5), which are followed by a short concluding chapter (Ch. 6) and three appendices (App. A, App. B and App. C). A brief description of each section follows.

Chapter 2 first gives a general historical overview of the evidence for dark matter. It then builds a case for studying WIMPs, and it finishes by explaining the argument for dark matter self-interactions, providing a quantified range of possible interaction strengths.

Chapter 3 establishes the model we use to explain the self-interactions and goes on to lay down the amplitudes for the scattering and annihilation processes. By employing the scattering amplitudes to derive the scattering cross section, a relationship between the dark matter fermion mass, the mediator mass and the mediator-fermion coupling constant is obtained within a region set by the range of allowed interaction strengths provided in Ch. 2 .

Chapter 4 explains the concept of freeze-out. It is shown that the annihilation cross section is required in order to perform a freeze-out calculation, and so a thermally averaged version of the annihilation cross section is derived. In order to achieve the correct relic abundance of dark matter, it is demonstrated that the previously-mentioned coupling constant must depend on the fermion's mass. This information is ultimately used to eliminate the coupling constant from the relationship obtained at the end of Ch. 3, and thereby to provide a strict relationship between the fermion mass and the mediator mass. The mediator mass turns out to be very light, and so it be- 
comes apparent that a low-velocity approximation made in Ch. 3 needs to be revisited. After correcting for the poor approximation, it is shown that the fermion mass has a maximum range imposed by a theoretical upper bound on the coupling constant, limiting the allowed mass for both the fermion and the mediator.

Chapter 5 describes the effect of introducing mixing between the mediator and the Higgs. Justification is provided for leaving the mixing unaddressed in previous chapters, and an allowed region of lifetimes and masses for the mediator is obtained by way of experimental constraints from Higgs decays. This, combined with a maximum mediator lifetime set by Big Bang nucleosynthesis, sets a lower bound on the mediator mass, which in turn may be used to set a lower bound on the dark matter fermion mass.

Chapter 6 is a concluding chapter that summarizes the main results and ties everything together.

Appendix A explains natural units, the units primarily used in this thesis, and relates them to the more well-known SI units.

Appendix B outlines the Runge-Kutta computational method and elaborates on how it is applied in Ch. 4.

Finally, App. C provides expressions for a pair of difficult integrals in Ch. 4. 


\section{Chapter 2}

\section{Dark Matter}

For the better part of a century, it has been clear that the majority of matter in the universe cannot be seen directly; rather, its existence may only be inferred by virtue of its gravitational effect upon that which is visible. Such a state of affairs is not without precedent, for during the nineteenth century, an eighth planet, along with its location, was predicted thanks to the otherwise inexplicable behaviour of Uranus [10]. It was clear that some undiscovered body was gently tugging upon the gas giant, and soon after the prediction of the eighth planet's location was made, Neptune was discovered. In similar fashion, dark matter is thought to account for what would otherwise be a series of large-scale gravitational anomalies. Unlike Neptune, however, evidence suggests that dark matter is made up not of baryons, but of some as-yet undiscovered particle or particles that, thanks to their extremely feeble interactions, are likely all but impossible to detect experimentally. 


\section{$2.1 \quad$ Evidence}

In the early 1930s, astronomers knew that celestial bodies existed outside the Milky Way galaxy, but the exact nature of those bodies had not yet been determined. At the time, the faint collections of stars and dust were called extragalactic nebulae [11], and though some believed they were galaxies just like our own, no one knew for sure. It was with great vigour, then, that the scientific community made use of different investigative techniques in an effort to uncover the nebulae's secrets.

One such technique was astronomical spectroscopy. Spectroscopy works by noting that a given gas, such as hydrogen, absorbs and emits only particular wavelengths of light. This information may subsequently be used to determine the chemical composition of some sample material. For example, say there is a distant star with a relatively cool outer-atmosphere composed primarily of hydrogen. The interior of the star essentially behaves as a black body, emitting the full spectrum of light. While it may be true that most of the luminosity is centred within a fairly narrow range of wavelengths as determined by the star's temperature, and that the luminosity decays for increasing or decreasing wavelengths, nowhere in this spectrum should the luminosity discontinuously drop. However, as the light leaves the star's interior and passes through the atmosphere, some particular wavelengths, which correspond to hydrogen's absorption spectrum, are absorbed, leaving "holes" for observers to detect here 
on Earth $[12,13]$. In this way, it is possible to determine the chemical composition of stars' atmospheres.

While being able to describe what chemical elements exist in a far-off celestial body - and in what proportions - is in itself a marvellous achievement, the astronomers of the early twentieth century could do even more with spectroscopy. As previously stated, gasses have their own signatures in the form of absorption and emission spectra. Taking again the example of the star with the hydrogen atmosphere, imagine that the star moves directly away from Earth at some velocity, $v$. Then the Doppler effect comes into play, and the observed wavelengths, $\lambda_{\text {obs }}$, corresponding to the absorption spectrum of hydrogen, will be shifted from the source wavelengths, $\lambda_{\mathrm{src}}$, as detailed in the following formula [14]:

$$
\lambda_{\mathrm{obs}}=\lambda_{\mathrm{src}} \sqrt{\frac{1+\frac{v}{c}}{1-\frac{v}{c}}},
$$

where $c$ is the speed of light in a vacuum. By recognizing that the stellar atmosphere displays hydrogen's absorption spectrum, just shifted away from what would be the case were the star stationary relative to Earth (see Fig. 2.1 ), it is possible to find the star's velocity. This procedure is typically made more complicated by the fact that systems do not, in general, move directly away from Earth; but the principle remains the same: the Doppler shifted spectra of distant bodies may be used to determine the relative velocities of those bodies. 


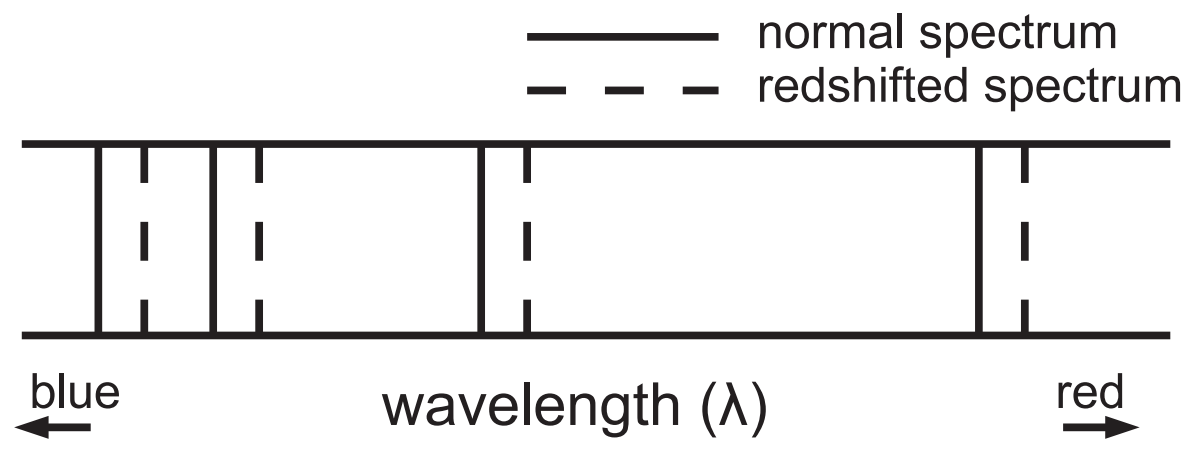

Figure 2.1: A pictorial representation of hydrogen's absorption lines. The solid lines represent the spectral signature in the reference frame of the source, whereas the dashed lines represent the spectral signature as seen by an observer. In this case, the source is receding from the observer, so the received light becomes stretched by the Doppler effect, resulting in longer wavelengths. This is known as a redshift (as opposed to a blueshift). The disparity between the solid and dashed lines may be used to calculate the velocity of the source relative to the observer.

After having employed the technique of astronomical spectroscopy, Fritz Zwicky published a paper in 1933 [11] detailing his findings concerning a group of approximately 800 extragalactic nebulae, which scientists now know to be galaxies, called the Coma Cluster. He found that in order for the galaxies to be moving at their recorded velocities, there would need to be more than 400 times as much mass within the cluster than the mass that could be attributed to the visible stars. He was therefore able to state that dark matter, matter that apparently does not radiate, must make up a much greater proportion of the universe than does luminous matter.

Since then, observations have continued to support Zwicky's claim that most of the matter in the universe is invisible to us and only detectable by virtue of its gravitational imprint. This state of affairs is perhaps nowhere so 
evident as in the form of a galactic rotation curve, a plot of the tangential velocity, $v_{t}$, of stars orbiting a host galaxy as a function of their distance, $r$, from its centre. If $M$ is the mass of the galaxy and $m$ is the mass of one of the stars that lies beyond the galactic disk (see Fig. 2.2), then $v_{t}$ should be readily calculable by applying Newton's Laws. (Of course, the same laws work inside the galactic disk, but within the $\operatorname{disk} M$ is variable and proportional to the mass that lies within the star's orbit.) The gravitational force on the star, $F_{g}$, is thus given by

$$
F_{g}=\frac{G M m}{r^{2}}
$$

where $G$ is the gravitational constant of the universe. Because the star undergoes (approximately) circular motion, it may be written that

$$
m \frac{v_{t}^{2}}{r}=\frac{G M m}{r^{2}}
$$

Rearranging yields

$$
v_{t}=\sqrt{\frac{G M}{r}},
$$

implying that

$$
v_{t} \propto \frac{1}{\sqrt{r}}
$$

The above result, which suggests that $v_{t}$ should fall off as $1 / \sqrt{r}$ for large $r$, stands in sharp contrast with the observed result, where $v_{t}$ stays roughly constant or even increases for increasing $r$. (A typical example may be seen 


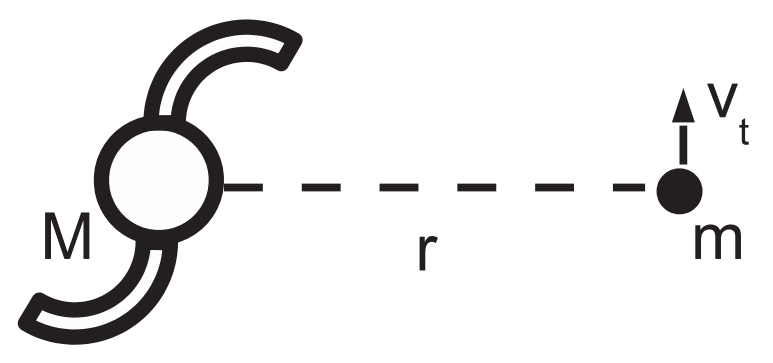

Figure 2.2: A diagram showing the pertinent elements for rotational curves. On the left is a galaxy of mass $M$, and on the right is a star of mass $m$. The orbital velocity of the star, $v_{t}$, is a function of the star's distance from the galactic centre, $r$.

in Fig. 2.3.) Consequently, it appears that dark matter is bound to galaxies in spherical halos that extend well beyond the galaxies' visible outer reaches $[15,16,17]$.

Another technique that has been used to map out dark matter is gravitational lensing. Sufficiently massive objects bend light to a detectable degree. Suppose there is some particularly dense region of dark matter that lies between Earth and a bright star. Then the dark matter behaves in a fashion similar to a lens, bending the light from the star behind it and focusing it onto astronomers' telescopes. Depending on the configuration of the system, observers may see multiple images of the distant star, typically in the form of bright arcs. By using Einstein's results from general relativity, then, it is possible to discern the mass and distribution of the dark matter "lens" [18]. An example of gravitational lensing is provided in Fig. 2.4.

These various observational methods have helped to further quantify the 


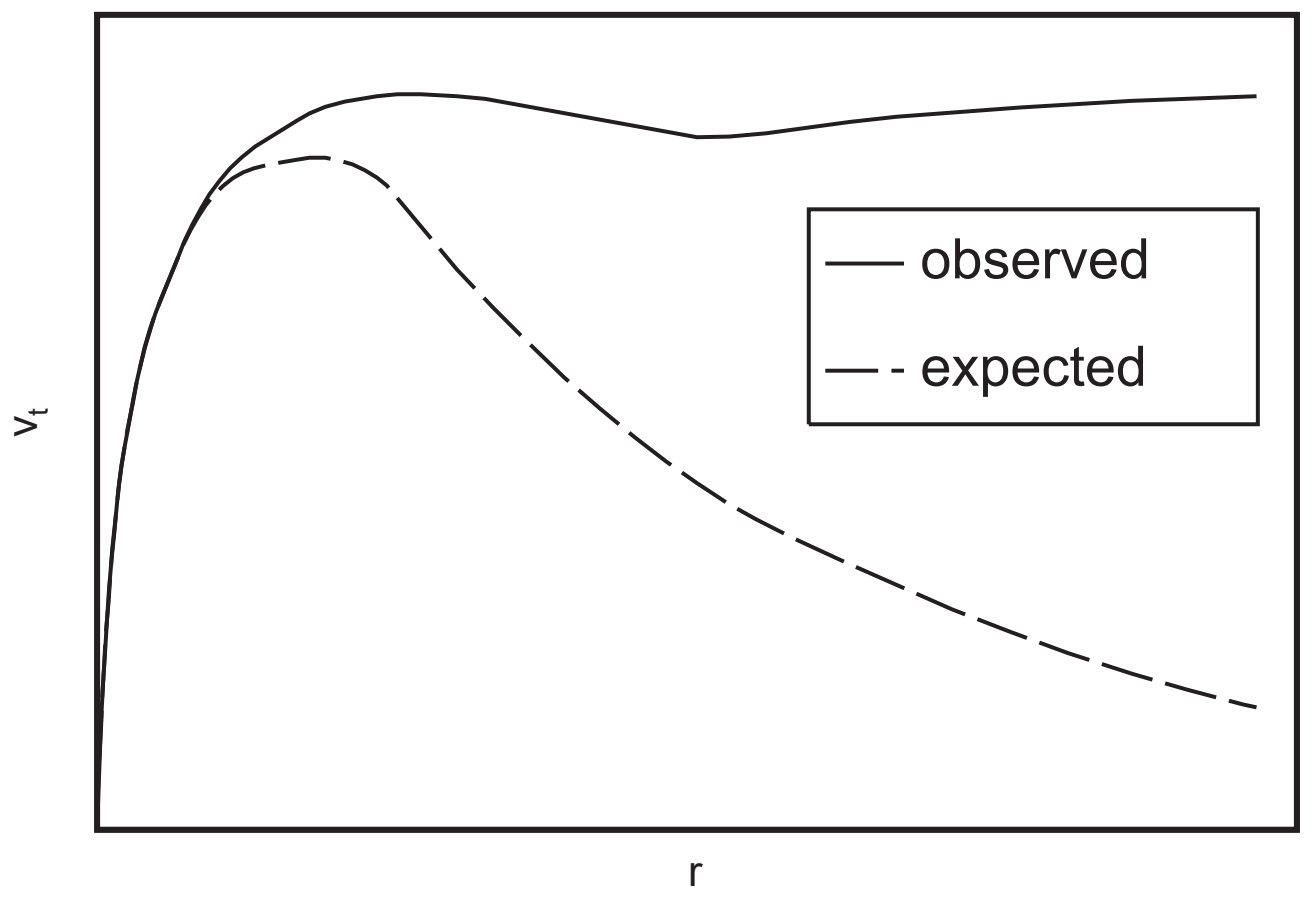

Figure 2.3: Typical contrast between the observed rotation curve of a galaxy in the presence of dark matter (solid line) and the expected rotation curve in its absence (dashed line).

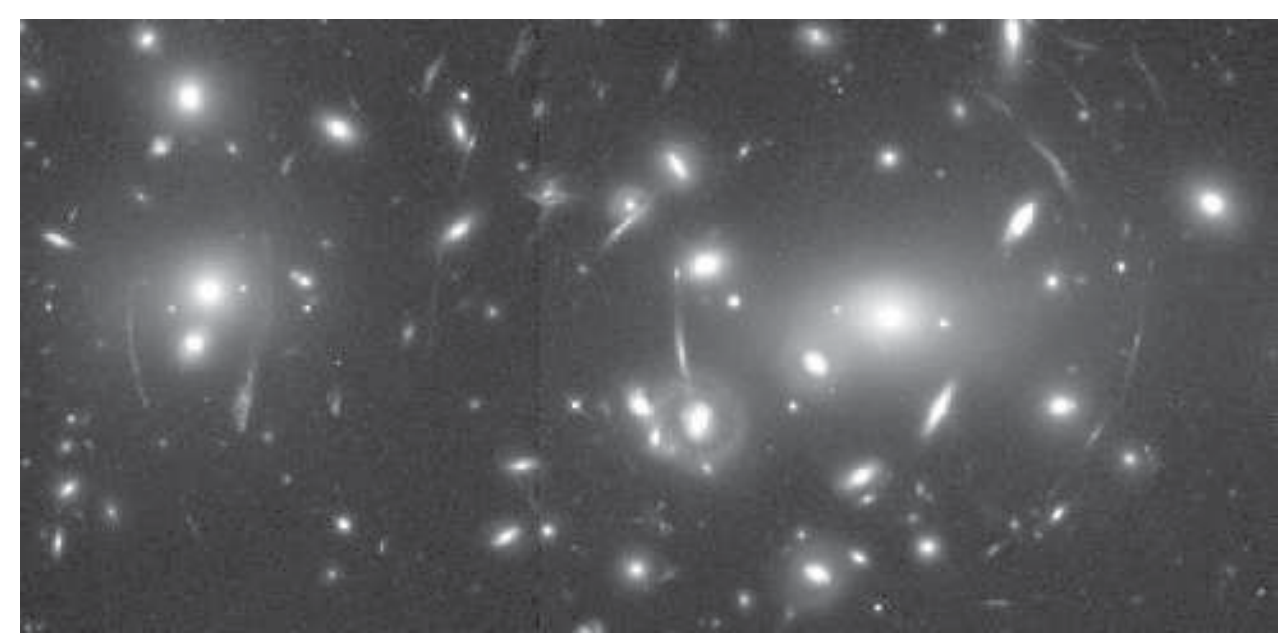

Figure 2.4: An example of gravitational lensing from NASA [19]. Distant galaxies are lensed by a foreground galaxy cluster and consequently show up as bright arcs. 
amount of dark matter in the universe. Roughly, it can be said that the universe is composed of only $5 \%$ regular, baryonic matter, while a full $26 \%$ is in the form of dark matter [15]. The remaining $69 \%$ is in the form of dark energy, which is responsible for the accelerated expansion of the universe [20]. (The dark part of dark energy is meant to elicit the same sense of bewilderment about the phenomenon as does the same word in dark matter, but that is where the similarities end.)

\subsection{Composition}

It has been established that most of the matter in the universe is dark. Moreover, the distribution of dark matter within the universe has been wellcatalogued. Despite these findings, however, the precise nature of dark matter remains a mystery. Theories attempting to explain what might actually comprise dark matter abound, but physical proof in support of these theories is markedly lacking.

In broad terms, there are three main possibilities when it comes to dark matter composition. The first is that it does not exist at all. While most physicists postulate that dark matter must fill the universe in order to account for its perceived gravitational effects, some say the problem lies with gravity itself. Thus there have been multiple proposed modified theories of gravity, each endeavouring to explain phenomena such as galactic rotation curves, 
merging cluster dynamics and otherwise inexplicable examples of gravitational lensing. As observational evidence has increased, many modified theories of gravity have necessarily been left behind, but unless dark matter is explicitly identified through some uncontroversial experiment, there will likely always be a modified theory of gravity as an alternative [16].

At present, one such theory is Scalar-Tensor-Vector-Gravity (or STVG) [21], which contends that General Relativity is not applicable at larger scales, specifically scales beyond the size of individual star systems. At the heart of the theory is the notion that gravity is actually much stronger than described in General Relativity, but that there is also a repulsive gravitational force mediated by a very light, spin-1 vector field. This repulsive gravitational field masks the attractive one at smaller scales, effectively making the attractive gravitational force appear weaker than it really is. This theory and others like it, however, typically suffer from difficulty explaining gravitational lensing.

Moving away from modified theories of gravity, the second possibility for dark matter is that it is just regular baryonic matter (matter made up of protons and neutrons) that is too cold to be readily detected by telescopes on Earth. This matter could take the form of dust, very faint stars or even planets that are not bound to star systems. As it turns out, however, this explanation has been largely discredited thanks to cosmological evidence [16, 22]. For one, in order to arrive at the large-scale structure of the universe as it is today, it seems that non-baryonic dark matter is required; for if the dark matter of the 
early universe were replaced by baryonic matter, the baryonic matter would not start clumping until relatively late in the universe's evolution, thereby slowing the structure-formation process and leaving the universe looking quite different than it does today. Furthermore, the amount of deuterium in the universe suggests that dark matter cannot be baryonic. To wit, deuterium is only destroyed and is never created in astrophysical processes. In addition, the amount of deuterium in the early universe should be proportional to the overall amount of baryonic matter. Since deuterium is destroyed at a known rate, it follows that too much baryonic matter in the early universe means too much deuterium at present. These arguments, combined with other cosmological principles, make it very unlikely that dark matter is made up of baryons [22].

That leaves the final possibility for dark matter, that it is made up of particles, typically WIMPs (Weakly Interacting Massive Particles). These are theoretical particles that would account for most, if not all, of the dark matter in the universe. Clearly WIMPs would have to be electrically neutral, lest they would be detectable by virtue of their absorption and emission of electromagnetic waves [20]. They would thus interact only via the weak force through the exchange of $W$ and $Z$ bosons. Alternatively, they might exchange some as-yet undiscovered mediator that would allow for a similar strength of interaction. If this latter option is the case, a whole so-called dark sector might exist [23]. It is informative here to consider the discovery of the neutrino, a very light, uncharged lepton that only interacts with other particles via the weak force. 
In 1931, Pauli was studying beta decays, and he noted that the laws of conservation of energy and momentum seemed to be violated in the beta decay process. He therefore hypothesized the existence of a particle that would participate in the decay and correct for any missing energy and momentum. This particle was later named the neutrino, but its actual experimental discovery was still decades away [14].

There are parallels to be drawn here with dark matter. Just as in the case of the neutrino, a law of the universe appears to be violated. In the case of the neutrino, the affront came in the form of violated conservation laws; for dark matter, it is General Relativity that seems to misbehave. It is quite possible, then, that WIMPs might fill in the gaps, just as did neutrinos in the case of beta decays. Indeed, one could ask whether neutrinos might themselves solve the dark matter problem. After all, neutrinos are weakly-interacting, and they are massive, if only slightly. Just as in the case of baryonic matter, however, well-grounded cosmological models show that such a large number of neutrinos in the early universe would lead to a universe with very different properties from what we observe today. Furthermore, because neutrinos are so light, they move at relativistic speeds, and it is impossible that such particles would settle in galactic halos and in other dark matter structures [16, 22].

Of course, there is the remote possibility that dark matter does not interact in any way save via the gravitational force, but then discovering it experimentally would be nearly impossible [20]. Thus it seems reasonable for 
particle physicists to assume that dark matter is composed of WIMPs and to make predictions accordingly.

\section{$2.3 \quad$ Self-Interactions}

Direct detection experiments have placed tight upper bounds on the strength of interaction between dark matter particles and particles of the Standard Model. However, there is no reason that dark matter cannot interact with itself to some appreciable degree within the dark sector [1].

Even so, upper bounds have been placed on such interactions. Consider the Bullet Cluster, which consists of a pair of galaxy clusters that passed through one another millions of years ago. It is evident upon examination of the clusters that during their collision, much of the intergalactic gas and dust contained in each was stripped away as it impacted the gas and dust of the other. The result is a pair of clusters conspicuously lacking the intergalactic medium one would expect to find. Instead, each cluster has a massive region of plasma trailing behind it [24].

Now, if dark matter self-interactions were as strong as the self-interactions of the clusters' intergalactic media, one would expect to find the dark matter distributions coinciding with the trailing, visible masses. Conversely, if the dark matter self-interactions were weaker, but not so weak that the dark matter halos would stay on top of the clusters' galaxies, then one would expect 
to detect the invisible mass somewhere between the galaxies and the trailing masses. Instead, it appears that the dark matter halos remain unseparated from the gas-stripped galaxies to within a certain degree of certainty. This in and of itself provides excellent evidence that dark matter is made up of WIMPs, for many modified theories of gravity have a hard time explaining why the gas clouds, which should make up most of the mass of the system, contain so much less mass than the stripped galaxies. In any event, the fact that the dark matter and stripped galaxies remain physically unseparated in the Bullet Cluster puts an upper limit on the self-interaction cross section (a measure of interaction likelihood) of dark matter, which may be quantified by stating that $[24]$

$$
\sigma / m \lesssim 1 \mathrm{~cm}^{2} / \mathrm{g}
$$

where $\sigma$ is the self-interaction cross section and $m$ is the mass of the dark matter particle.

On the other hand, in contrast with the trend of evidence suggesting that dark matter either does not self-interact or does so quite weakly, recent observations of the cluster Abell 3827 have tentatively demonstrated the existence of measurable dark matter self-interactions [1]. The observed region consists of four elliptical galaxies, each with its own dark matter halo. The galaxies are falling through a region with an appreciable dark matter background, and it is expected that, should self-interactions occur, a drag force should tug 


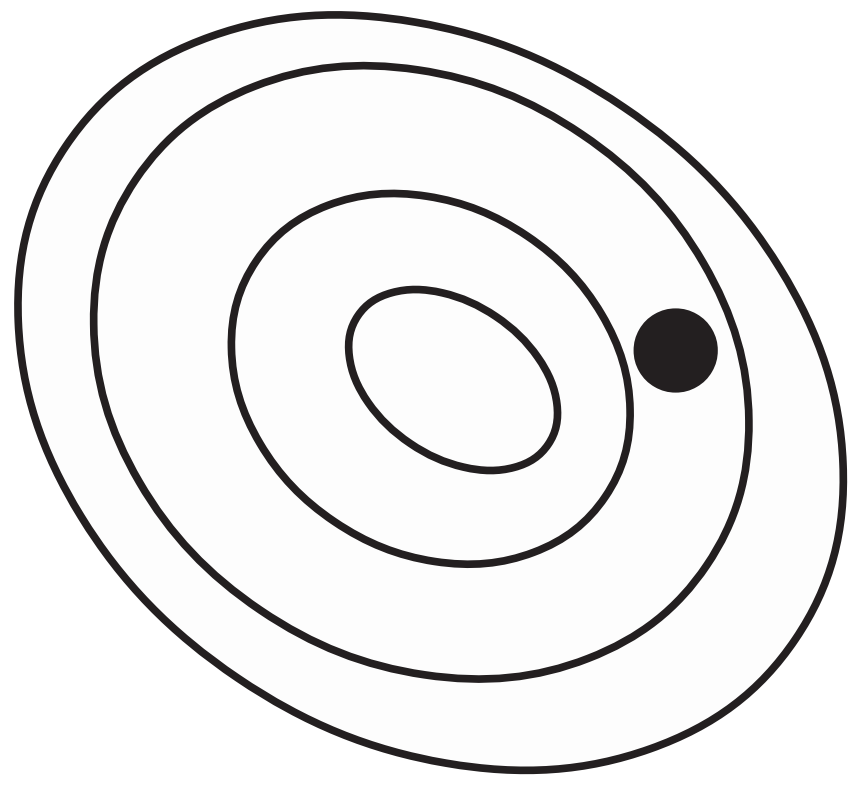

Figure 2.5: A schematic representation of the findings from Ref. [1]. The black dot represents one of the four elliptical galaxies. Note how the contour lines, which depict regions of equal mass density, converge on a point to the left of the galaxy's location.

upon the dark matter halos, thereby leading to a spatial offset between the halos and their host galaxies. This effect was documented through the use of gravitational lensing techniques, and is pronounced in at least one of the four galaxies. A schematic representation of the effect is provided in Fig. 2.5. This observation dictates that if short-range self-interactions are responsible for the offset, then dark matter should have a scattering cross section falling within the range [2]

$$
1.0 \mathrm{~cm}^{2} / \mathrm{g} \lesssim \sigma / \mathrm{m} \lesssim 1.5 \mathrm{~cm}^{2} / \mathrm{g}
$$

which does not agree with Eq. (2.3), but at least does not exceed the limit by orders of magnitude. 
There are other possible explanations for the observations of Abell 3827. Most notably, it could be that increased star formation is occurring in regions of compacted gas, causing an average off-centre brightness within the galaxies [1]. However, if the effect is truly the result of dark matter self-interactions, it will have far-reaching consequences in the fields of astronomy and particle physics. 


\section{Chapter 3}

\section{A Candidate Model}

The apparent dark matter self-interactions within Abell 3827 [1] demand explanation. We wish to demonstrate whether a simple dark matter model might account for the observations while also surviving various cosmological and experimental constraints. To that end, no set of quantities is more important than the interaction cross sections, a set of quantities that quantify the likelihood of scattering and annihilation events. Armed with these cross sections, it will ultimately be possible to either reject our model outright or to show that it might be representative of the natural world somewhere within a specified parameter space; but, before that can happen, the model must be built and the cross sections derived. 


\subsection{Particles and Feynman Rules}

We propose a relatively simple model for dark matter in which the candidate particle has a spin of one half and is denoted by $\chi$. The fact that it is a spin- $\frac{1}{2}$ particle implies that it is a fermion, just like baryons, leptons and quarks - the fundamental bits of matter in the Standard Model. Furthermore, we specify that the $\chi$ is a Dirac fermion, meaning its antiparticle is distinct from itself (as opposed to a Majorana fermion). In keeping with conventions, we name this antiparticle $\bar{\chi}[20]$.

In order to account for the observed self-interactions, we add a mediator to our model, a scalar boson (spin 0) denoted by $\phi$. Though the known mediators - gluons, photons and $W$ and $Z$ bosons - are all vector bosons [20], we decide to make the $\phi$ a scalar for simplicity and so that it might mix with the Higgs. In doing so, our dark matter is given a pathway to annihilate to Standard Model particles, thereby allowing us to model its thermal freeze-out [15], a process that shall be discussed further in Ch. 4. Together the $\chi, \bar{\chi}$ and $\phi$ make up the dark sector. This model is similar to one developed in Ref. [9], though, unlike in Ref. [9], we have the express goal of accommodating self-interactions.

It is now prudent to enumerate the ways in which these particles are allowed to interact with one another. To wit, consider the Lagrangian density for fields, $\mathcal{L}$. Classically, the Lagrangian is a quantity that, when incorporated 
into the Euler-Lagrange equation, allows one to derive the equations of motion for a system. The methodology is equivalent to analysis using Newtonian mechanics, but often the Lagrangian route is the simpler of the two. When dealing with fields, the equivalent of the Euler-Lagrange equation is

$$
\frac{\partial}{\partial \mu}\left(\frac{\partial \mathcal{L}}{\partial\left(\frac{\partial a_{i}}{\partial \mu}\right)}\right)=\frac{\partial \mathcal{L}}{\partial a_{i}},
$$

where $\mu$ is a spacetime coordinate and $a_{i}$ is the $i$ th field. When it comes to interactions, the most useful Lagrangian that can be written for our model is the dark matter interaction Lagrangian density, $\mathcal{L}_{\mathrm{DM}}$ int, which is given by

$$
\mathcal{L}_{\mathrm{DM} \text { int }}=-g \bar{\chi} \chi \phi-\frac{1}{3 !} \tilde{g} \phi \phi \phi
$$

where $\chi, \bar{\chi}$ and $\phi$ are the fields that generate their respective particles and $g$ and $\tilde{g}$ are coupling constants. Note that the 3 ! in the denominator of the second term is included for convenience because there are $3 !=6$ ways of arranging three identical $\phi$ particles in a three-way interaction such that the overall process looks identical. To illustrate, imagine that one high-energy $\phi$ decays into two lower-energy $\phi$ s. If the ingoing $\phi$ is labelled $\phi_{1}$ and the two outgoing $\phi$ s are labelled $\phi_{2}$ and $\phi_{3}$, then switching the labels around in any of the six possible ways has no bearing whatsoever on the process; the labels are utterly interchangeable. 
The dark matter interaction Lagrangian density of Eq. (3.2) is needed in order to draw the Feynman diagrams for this model, and Feynman diagrams are integral to the calculation of cross sections for scattering events. Far from simple schematics of particle reactions, Feynman diagrams may be decoded into precise mathematical statements through the application of the relevant Feynman rules. These mathematical statements may subsequently be used to calculate the amplitude, $\mathcal{M}$, of a reaction, which is a crucial aspect of cross section calculations.

Here it will be sufficient to list the Feynman rules that arise through consideration of the particle types in our proposed model, as well as the interactions apparent upon investigation of Eq. (3.2). (See Ref. [25] for details.) The Feynman rules are shown below:

1. Propagators

$$
\begin{aligned}
& \text { scalar : } \quad---\stackrel{q}{\longrightarrow---}=\frac{i}{q^{2}-m_{\phi}^{2}} \\
& \text { fermion : } \longrightarrow \frac{p}{\longrightarrow}=\frac{i\left(\not p+m_{\chi}\right)}{p^{2}-m_{\chi}^{2}}
\end{aligned}
$$

2. Vertices 

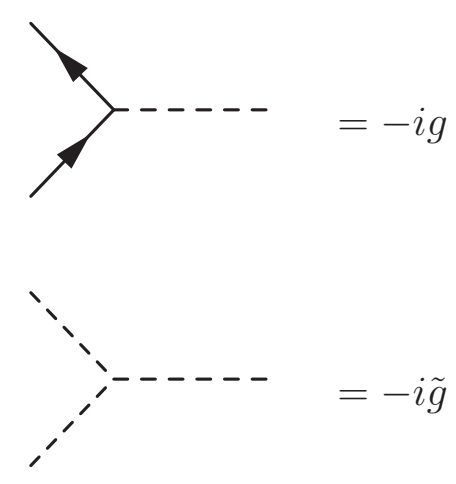

3. External Legs
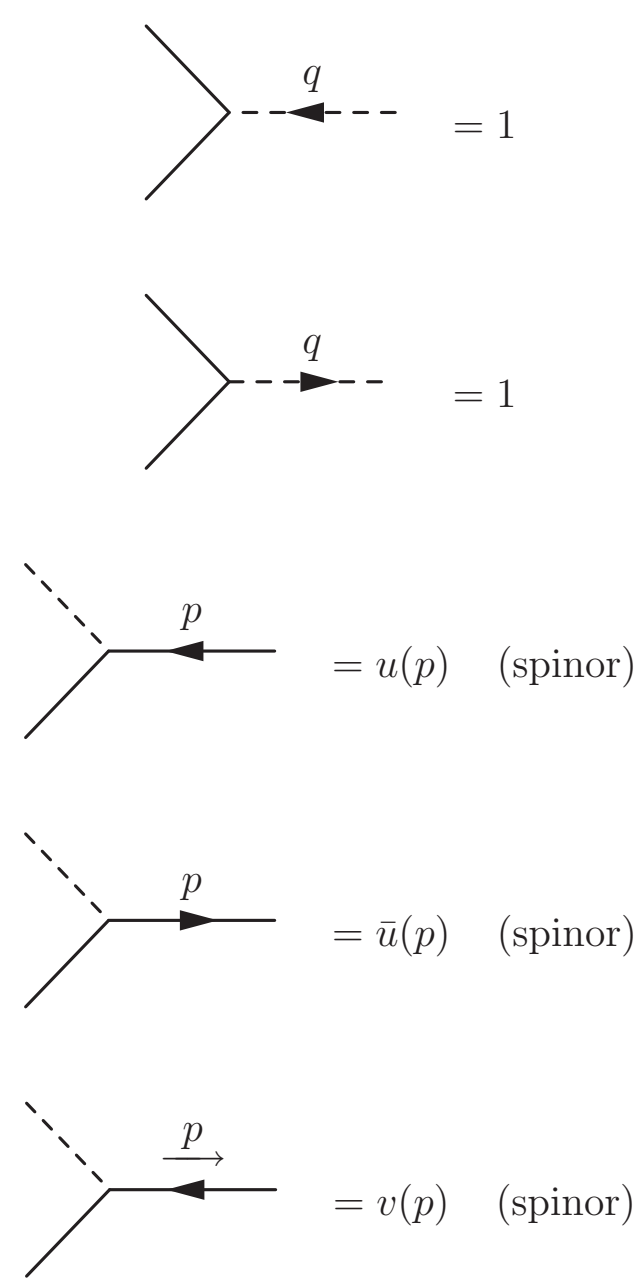


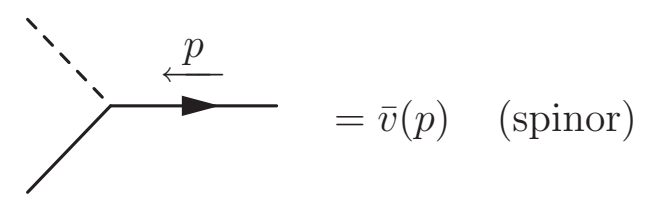

The above list contains several symbols that demand explanation. Firstly, $i$ is the imaginary unit such that $i=\sqrt{-1}$. Secondly, $p$ and $q$ are four-momenta defined in such a way that if particle $A$ has energy $E_{A}$ and momentum $\mathbf{p}$ and particle $B$ has energy $E_{B}$ and momentum $\mathbf{q}$, then $p=\left(E_{A}, \mathbf{p}\right)$ and $q=$ $\left(E_{B}, \mathbf{q}\right)$. Thirdly, $m_{\chi}$ and $m_{\phi}$ are the masses of the $\chi$ (or equivalently the $\bar{\chi}$ ) and the $\phi$, respectively. Fourthly, the spinors are four-component objects that encapsulate the spin part of the free-particle solutions to the Dirac equation, the quantum mechanical equation that governs free fermions. Furthermore, the $u$ spinors correspond to particles, and the $v$ spinors correspond to antiparticles. Finally, the "slash" notation has the following interpretation:

$$
\not p=p_{\mu} \gamma^{\mu}
$$

where Einstein summation convention is followed and the gamma matrices 
are defined as follows:

$$
\begin{aligned}
\gamma^{0} & =\left[\begin{array}{cccc}
1 & 0 & 0 & 0 \\
0 & 1 & 0 & 0 \\
0 & 0 & -1 & 0 \\
0 & 0 & 0 & -1
\end{array}\right] \quad \gamma^{1}=\left[\begin{array}{cccc}
0 & 0 & 0 & 1 \\
0 & 0 & 1 & 0 \\
0 & -1 & 0 & 0 \\
-1 & 0 & 0 & 0
\end{array}\right] \\
\gamma^{2} & =\left[\begin{array}{cccc}
0 & 0 & 0 & -i \\
0 & 0 & i & 0 \\
0 & i & 0 & 0 \\
-i & 0 & 0 & 0
\end{array}\right] \quad \gamma^{3}=\left[\begin{array}{cccc}
0 & 0 & 0 \\
0 & 0 & 0 & -1 \\
-1 & 0 & 0 & 0 \\
0 & 1 & 0 & 0
\end{array}\right] .
\end{aligned}
$$

It is simpler to write the $4 \times 4$ gamma matrices in a compact $2 \times 2$ format by making use of the Pauli spin matrices:

$$
\gamma^{0}=\left[\begin{array}{cc}
1 & 0 \\
0 & -1
\end{array}\right] \text { and } \gamma^{i}=\left[\begin{array}{cc}
0 & \sigma^{i} \\
-\sigma^{i} & 0
\end{array}\right]
$$

where $i$ runs from 1 to 3 . It is also useful here to define $\gamma^{5}[20,25]$ such that

$$
\gamma^{5}=i \gamma^{0} \gamma^{1} \gamma^{2} \gamma^{3}
$$




\subsection{Amplitudes}

It has now twice been stated that the cross section for a given particle interaction quantifies the likelihood of that interaction's occurrence, but a precise explanation has not been given, so at this point an exact definition is in order. If there are two beams of particles, beam $A$ and beam $B$, that are heading toward one another, and if beam $A$ has particle density $\rho_{A}$ and length $l_{A}$ and beam $B$ has particle density $\rho_{B}$ and length $l_{B}$, and furthermore if $\mathcal{A}$ is the overlapping area shared by the two beams and $N$ is the number of scattering events that occur, then the cross section of the interaction is given by $\sigma$, where $[25]$

$$
\sigma=\frac{N}{\rho_{A} \rho_{B} l_{A} l_{B} \mathcal{A}}
$$

To simplify matters, consider only cases with two final-state particles. Because the products emerge with variable momenta, $\sigma$ can only be determined by way of the differential cross section

$$
\frac{d \sigma}{d^{3} p_{1} d^{3} p_{2}}
$$

where $p_{1}$ and $p_{2}$ are the momenta of the outgoing particles. This quantity may be further constrained by noting that conservation laws allow only certain energies and momenta to emerge, and so the only truly free parameters are the

polar and azimuthal angles for one of the particles, $\theta$ and $\phi$. The quantity of 
interest, then, is $\frac{d \sigma}{d \Omega}$, where $d \Omega=\sin \theta d \theta d \phi$ is the solid angle differential. For the special case of two ingoing particles with four-momenta $p$ and $p^{\prime}$ and two outgoing particles with four-momenta $k$ and $k^{\prime}$, the differential cross section is given by [25]

$$
\left(\frac{d \sigma}{d \Omega}\right)_{\mathrm{CM}}=\frac{1}{2 E_{p} 2 E_{p^{\prime}}\left|v_{p}-v_{p^{\prime}}\right|} \frac{|\mathbf{k}|}{(2 \pi)^{2} 4 E_{\mathrm{CM}}}\left|\mathcal{M}_{p, p^{\prime} \rightarrow k, k^{\prime}}\right|^{2},
$$

where the CM subscript implies that the system should be examined in the centre-of-mass frame, $E_{p}$ and $E_{p^{\prime}}$ are the energies of the ingoing particles, $v_{p}$ and $v_{p^{\prime}}$ are the velocities of those same particles, $E_{\mathrm{CM}}$ is the centre-of-mass energy, $|\mathbf{k}|$ is the magnitude of the momentum of an outgoing particle and $\mathcal{M}$ is the amplitude of the process.

Upon inspection of Eq. (3.9), it would appear that calculating cross sections is a trivial matter; but, quite to the contrary, finding $\mathcal{M}$ is a lengthy and oftentimes difficult undertaking. It is therefore prudent to go through one amplitude calculation in fine detail. Subsequent derivations, however, will be skipped in an effort to avoid unnecessary tedium, and only the main results will be shown. Furthermore, explicit cross section expressions will not be derived until necessary because their complete calculation often involves approximations. The amplitudes for the processes $\chi \chi \rightarrow \chi \chi, \bar{\chi} \chi \rightarrow \bar{\chi} \chi$ and $\bar{\chi} \chi \rightarrow \phi \phi$, however, will be calculated analytically insofar as is possible in this section. That way, the amplitudes in this section may be used for future 
reference without the need to recall which approximations were and were not involved.

\section{Amplitude for $\chi \chi \rightarrow \chi \chi$}

The first step in calculating this amplitude is to find the quantity $i \mathcal{M}$, which is the sum of the relevant Feynman diagrams [25]. Labels $p, p^{\prime}, k, k^{\prime}, q$ and $q^{\prime}$ indicate particle momenta, and we adopt the convention that time runs upward in all diagrams. The two, summed diagrams for the $\chi \chi \rightarrow \chi \chi$ process are shown below:

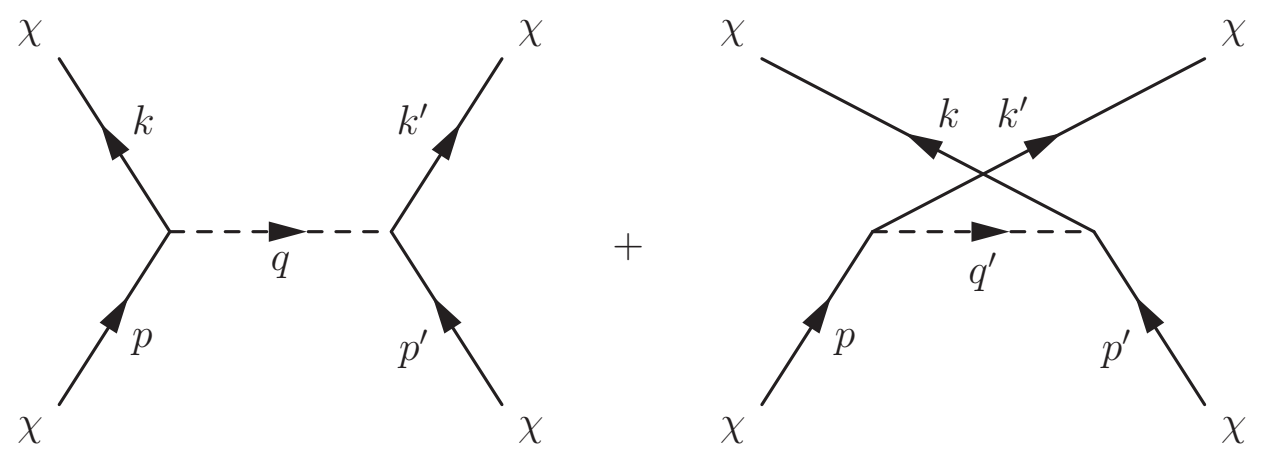

To convert the diagrams into mathematical expressions, one must run along the fermion lines against the directional arrows, writing down the corresponding Feynman rules from Sec. 3.1 for the lines and vertices as they appear and multiplying them together in that order [25]. Thus,

$$
\begin{aligned}
i \mathcal{M}= & -\bar{u}(k)(-i g) u(p) \frac{i}{q^{2}-m_{\phi}^{2}} \bar{u}\left(k^{\prime}\right)(-i g) u\left(p^{\prime}\right) \\
& +\bar{u}\left(k^{\prime}\right)(-i g) u(p) \frac{i}{q^{\prime 2}-m_{\phi}^{2}} \bar{u}(k)(-i g) u\left(p^{\prime}\right) .
\end{aligned}
$$


Note the negative sign in front of the first term. It was introduced to account for the fact that the outgoing lines in the diagrams are crossed relative to each other [25].

Performing some minor algebra on Eq. (3.10) and taking the absolute square of both sides leads to

$$
\begin{aligned}
|\mathcal{M}|^{2}=g^{4} & {\left[\frac{1}{\left(q^{2}-m_{\phi}^{2}\right)^{2}} \bar{u}(p) u(k) \bar{u}\left(p^{\prime}\right) u\left(k^{\prime}\right) \bar{u}(k) u(p) \bar{u}\left(k^{\prime}\right) u\left(p^{\prime}\right)\right.} \\
& +\frac{1}{\left(q^{\prime 2}-m_{\phi}^{2}\right)^{2}} \bar{u}(p) u\left(k^{\prime}\right) \bar{u}\left(p^{\prime}\right) u(k) \bar{u}\left(k^{\prime}\right) u(p) \bar{u}(k) u\left(p^{\prime}\right) \\
& -\frac{1}{\left(q^{2}-m_{\phi}^{2}\right)\left(q^{\prime 2}-m_{\phi}^{2}\right)} \bar{u}(p) u(k) \bar{u}\left(p^{\prime}\right) u\left(k^{\prime}\right) \bar{u}\left(k^{\prime}\right) u(p) \bar{u}(k) u\left(p^{\prime}\right) \\
& \left.-\frac{1}{\left(q^{2}-m_{\phi}^{2}\right)\left(q^{\prime 2}-m_{\phi}^{2}\right)} \bar{u}(p) u\left(k^{\prime}\right) \bar{u}\left(p^{\prime}\right) u(k) \bar{u}(k) u(p) \bar{u}\left(k^{\prime}\right) u\left(p^{\prime}\right)\right] .
\end{aligned}
$$

As it turns out, in order to match experiment, $|\mathcal{M}|^{2}$ is not actually substituted into Eq. (3.9). Rather, the quantity of interest is

$$
\frac{1}{2} \sum_{\text {spins } p} \frac{1}{2} \sum_{\text {spins } p^{\prime}} \sum_{\text {spins } k} \sum_{\text {spins } k^{\prime}}|\mathcal{M}|^{2},
$$

where the sums are run over the spins of the particles with the indicated momenta. This altered amplitude is meant to reflect experiment; the experimenter does not know which spin — up or down — the ingoing fermions carry, and so she averages over the ingoing spins. At the same time, she does not care what spins the outgoing particles carry, only that the reaction occurs and 
outgoing particles actually exist, so she sums over all outgoing particle events [25]. Adapting Eq. (3.11) accordingly yields

$$
\begin{aligned}
\frac{1}{4} \sum_{\text {spins }}|\mathcal{M}|^{2}= & \frac{g^{4}}{4} \sum_{\text {spins }} \sum_{\text {spins }} \sum_{p^{\prime}} \sum_{\text {spins }} \sum_{\text {spins } k^{\prime}}[ \\
& \frac{1}{\left(q^{2}-m_{\phi}^{2}\right)^{2}}(\bar{u}(p) u(k) \bar{u}(k) u(p))\left(\bar{u}\left(p^{\prime}\right) u\left(k^{\prime}\right) \bar{u}\left(k^{\prime}\right) u\left(p^{\prime}\right)\right) \\
& +\frac{1}{\left(q^{\prime 2}-m_{\phi}^{2}\right)^{2}}\left(\bar{u}(p) u\left(k^{\prime}\right) \bar{u}\left(k^{\prime}\right) u(p)\right)\left(\bar{u}\left(p^{\prime}\right) u(k) \bar{u}(k) u\left(p^{\prime}\right)\right) \\
& -\frac{1}{\left(q^{2}-m_{\phi}^{2}\right)\left(q^{\prime 2}-m_{\phi}^{2}\right)}\left(\bar{u}(p) u(k) \bar{u}(k) u\left(p^{\prime}\right) \bar{u}\left(p^{\prime}\right) u\left(k^{\prime}\right) \bar{u}\left(k^{\prime}\right) u(p)\right) \\
& \left.-\frac{1}{\left(q^{2}-m_{\phi}^{2}\right)\left(q^{\prime 2}-m_{\phi}^{2}\right)}\left(\bar{u}(p) u\left(k^{\prime}\right) \bar{u}\left(k^{\prime}\right) u\left(p^{\prime}\right) \bar{u}\left(p^{\prime}\right) u(k) \bar{u}(k) u(p)\right)\right] .
\end{aligned}
$$

The spinors in Eq. (3.12) have been rearranged into a more useful order by moving them in pairs, which is permitted because the spinors with bars are single-row matrices and the spinors without bars are single-column matrices. The form of Eq. (3.12) is useful because of the following two trace theorems [25]:

$$
\sum_{\text {spins } p} u(p) \bar{u}(p)=\not p+m_{\chi} \text { and } \sum_{\text {spins } p} v(p) \bar{v}(p)=\not p-m_{\chi}
$$

where it is implied that $m_{\chi}$ is multiplied by the $4 \times 4$ identity matrix. Note that the second equation does not come into play here. It is, however, used in the other amplitude calculations.

Moving on, it would be ideal if the first of Eqs. (3.13) could be immediately applied to Eq. (3.12). Unfortunately, the factors in the large parentheses, while 
almost in the necessary form, are not quite right. Specifically, each of these factors ends with a $u$ that needs to be somehow brought to the front so that all pairs of $u$ and $\bar{u}$ sharing the same four-momentum argument are side-by-side. This rearrangement cannot be done so long as the $u$ variables remain spinors, so indices are added to allow examination of individual terms. For brevity, consider just the first of the terms enclosed in parentheses in Eq. (3.12), along with its associated summations. It may be shown that

$$
\begin{aligned}
\sum_{\text {spins } p} \sum_{\text {spins } k} \bar{u}_{a}(p) \mathbf{1}_{a b} u_{b}(k) \bar{u}_{c}(k) \mathbf{1}_{c d} u_{d}(p) & \\
= & \sum_{\text {spins } p} u_{d}(p) \bar{u}_{a}(p) \mathbf{1}_{a b} \sum_{\text {spins } k} u_{b}(k) \bar{u}_{c}(k) \mathbf{1}_{c d} \\
= & \left(\not p+m_{\chi}\right)_{d a} \mathbf{1}_{a b}\left(\not k+m_{\chi}\right)_{b c} \mathbf{1}_{c d},
\end{aligned}
$$

where $\mathbf{1}$ is just the identity matrix. Given that the last line begins and ends with the same index, it is clear that it is just the sum of the diagonal components of the matrix it comprises. The sum of the diagonal components of a matrix is its trace, and the trace of a matrix $A$ is written $\operatorname{Tr}(A)$. Therefore, the factor enclosed in the first pair of parentheses in Eq. (3.12) may be written

$$
\operatorname{Tr}\left[\left(\not p+m_{\chi}\right)\left(\not k+m_{\chi}\right)\right]
$$


and, by extension, Eq. (3.12) becomes

$$
\begin{aligned}
& \frac{1}{4} \sum_{\text {spins }}|\mathcal{M}|^{2}= \\
& \frac{g^{4}}{4}\left\{\frac{1}{\left(q^{2}-m_{\phi}^{2}\right)^{2}} \operatorname{Tr}\left[\left(\not p+m_{\chi}\right)\left(\not k+m_{\chi}\right)\right] \operatorname{Tr}\left[\left(\not \not^{\prime \prime}+m_{\chi}\right)\left(\not k^{\prime \prime}+m_{\chi}\right)\right]\right. \\
& +\frac{1}{\left(q^{\prime 2}-m_{\phi}^{2}\right)^{2}} \operatorname{Tr}\left[\left(\not p+m_{\chi}\right)\left(\not k^{\prime}+m_{\chi}\right)\right] \operatorname{Tr}\left[\left(\not \not^{\prime \prime}+m_{\chi}\right)\left(\not k+m_{\chi}\right)\right] \\
& -\frac{1}{\left(q^{2}-m_{\phi}^{2}\right)\left(q^{\prime 2}-m_{\phi}^{2}\right)}\left(\operatorname{Tr}\left[\left(\not p+m_{\chi}\right)\left(\not k+m_{\chi}\right)\left(\not p^{\prime \prime}+m_{\chi}\right)\left(\not k^{\prime \prime}+m_{\chi}\right)\right]\right. \\
& \left.\left.+\operatorname{Tr}\left[\left(\not p+m_{\chi}\right)\left(\not k^{\prime \prime}+m_{\chi}\right)\left(\not p^{\prime \prime}+m_{\chi}\right)\left(\not k+m_{\chi}\right)\right]\right)\right\} \text {. }
\end{aligned}
$$

The next order of business is to work out the traces, a task made easier through careful exploitation of the following facts [25]:

$$
\begin{aligned}
\operatorname{Tr}(\mathbf{1}) & =4, \\
\operatorname{Tr}\left(\gamma^{\mu} \gamma^{\nu}\right) & =4 g^{\mu \nu} \text { and } \\
\operatorname{Tr}\left(\gamma^{\mu} \gamma^{\nu} \gamma^{\rho} \gamma^{\sigma}\right) & =4\left(g^{\mu \nu} g^{\rho \sigma}-g^{\mu \rho} g^{\nu \sigma}+g^{\mu \sigma} g^{\nu \rho}\right),
\end{aligned}
$$

where $g^{\mu \nu}$ is the Minkowski metric tensor such that

$$
g^{\mu \nu}=\left[\begin{array}{cccc}
1 & 0 & 0 & 0 \\
0 & -1 & 0 & 0 \\
0 & 0 & -1 & 0 \\
0 & 0 & 0 & -1
\end{array}\right] .
$$


In addition to the above rules, it should be further noted that any trace of an odd number of gamma matrices is automatically zero. Also, recall that $\not p=p_{\mu} \gamma^{\mu}$.

With all these definitions and rules established, it is now possible to find the traces in Eq. (3.16). The results are listed below:

$$
\begin{gathered}
\operatorname{Tr}\left[\left(\not p+m_{\chi}\right)\left(\not k+m_{\chi}\right)\right]=4\left(p \cdot k+m_{\chi}{ }^{2}\right), \\
\operatorname{Tr}\left[\left(\not p^{\prime \prime}+m_{\chi}\right)\left(\not k^{\prime \prime}+m_{\chi}\right)\right]=4\left(p^{\prime} \cdot k^{\prime}+m_{\chi}{ }^{2}\right), \\
\operatorname{Tr}\left[\left(\not p+m_{\chi}\right)\left(\not k^{\prime \prime}+m_{\chi}\right)\right]=4\left(p \cdot k^{\prime}+m_{\chi}{ }^{2}\right), \\
\operatorname{Tr}\left[\left(\not \not p^{\prime}+m_{\chi}\right)\left(\not k+m_{\chi}\right)\right]=4\left(p^{\prime} \cdot k+m_{\chi}{ }^{2}\right), \\
\operatorname{Tr}\left[\left(\not p+m_{\chi}\right)\left(\not k+m_{\chi}\right)\left(\not p^{\prime \prime}+m_{\chi}\right)\left(\not k^{\prime}+m_{\chi}\right)\right]=4\left[(p \cdot k)\left(p^{\prime} \cdot k^{\prime}\right)-\left(p \cdot p^{\prime}\right)\left(k^{\prime} \cdot k^{\prime}\right)\right. \\
\left.+\left(p \cdot k^{\prime}\right)\left(k \cdot p^{\prime}\right)+m_{\chi}{ }^{2}\left(p \cdot k^{\prime}+p^{\prime} \cdot p^{\prime}+p \cdot k+k^{\prime} \cdot p^{\prime}+k^{\prime} \cdot k+p^{\prime} \cdot k\right)+m_{\chi}{ }^{4}\right] \text { and } \\
\operatorname{Tr}\left[\left(\not p+m_{\chi}\right)\left(\not k^{\prime}+m_{\chi}\right)\left(\not p^{\prime \prime}+m_{\chi}\right)\left(\not k+m_{\chi}\right)\right]=4\left[\left(p \cdot k^{\prime}\right)\left(p^{\prime} \cdot k\right)-\left(p \cdot p^{\prime}\right)(k \cdot k)\right. \\
\left.+(p \cdot k)\left(k^{\prime} \cdot p^{\prime}\right)+m_{\chi}{ }^{2}\left(p \cdot k+p^{\prime} \cdot p^{\prime}+p \cdot k^{\prime}+k \cdot p^{\prime}+k \cdot k^{\prime}+p^{\prime} \cdot k^{\prime}\right)+m_{\chi}{ }^{4}\right] .
\end{gathered}
$$

It is particularly noteworthy here that the final two traces are identical.

Simple investigation of the left-most vertices of the two Feynman diagrams above Eq. (3.10) at the start of this section reveals that $q=p-k$ and $q^{\prime}=p-k^{\prime}$. In terms of the various four-momenta, then, the amplitude from 
Eq. (3.16) is

$$
\begin{aligned}
& \frac{1}{4} \sum_{\text {spins }}|\mathcal{M}|^{2}=2 g^{4}\left\{\frac{2\left(p \cdot k+m_{\chi}^{2}\right)\left(p^{\prime} \cdot k^{\prime}+m_{\chi}^{2}\right)}{\left[(p-k)^{2}-m_{\phi}^{2}\right]^{2}}\right. \\
& +\frac{2\left(p \cdot k^{\prime}+m_{\chi}^{2}\right)\left(p^{\prime} \cdot k+m_{\chi}^{2}\right)}{\left[\left(p-k^{\prime}\right)^{2}-m_{\phi}^{2}\right]^{2}}-\frac{\left(p \cdot k^{\prime}\right)\left(p^{\prime} \cdot k\right)-\left(p \cdot p^{\prime}\right)(k \cdot k)+(p \cdot k)\left(k^{\prime} \cdot p^{\prime}\right)}{\left[(p-k)^{2}-m_{\phi}^{2}\right]\left[\left(p-k^{\prime}\right)^{2}-m_{\phi}{ }^{2}\right]} \\
& \left.-\frac{m_{\chi}^{2}\left(p \cdot k+p^{\prime} \cdot p^{\prime}+p \cdot k^{\prime}+k \cdot p^{\prime}+k \cdot k^{\prime}+p^{\prime} \cdot k^{\prime}\right)+m_{\chi}{ }^{4}}{\left[(p-k)^{2}-m_{\phi}^{2}\right]\left[\left(p-k^{\prime}\right)^{2}-m_{\phi^{2}}{ }^{2}\right]}\right\}
\end{aligned}
$$

This is not the end of the story, however, for it is far more useful to work out cross section amplitudes in terms of energy, mass and scattering angles. It is therefore necessary to examine the kinematics of the scattering event. A diagram of a typical scattering event is shown in Fig. 3.1. Two $\chi$ particles with four momenta $p$ and $p^{\prime}$ collide in the centre-of-mass frame along the $z$ axis. It follows that, because the particles are identical, they have equal energies. Each particle thus has energy $E$, where $E_{\mathrm{CM}}=2 E$. The magnitude of the particles' momenta ought to be equal in this frame, so

$$
p=(E, 0,0,|\mathbf{p}|) \text { and } p^{\prime}=(E, 0,0,-|\mathbf{p}|) .
$$

After the particles interact, they fly off at an angle $\theta$ as depicted in Fig. 3.1 , where the $x$ axis is chosen so that $\theta$ lies in the $x$ - $z$ plane. In addition, conservation of energy dictates that the outgoing particles, which are of equal mass, should each have energy $E$. Moreover, because the two final-state particles have the same mass and energy as the two initial-state particles, they 


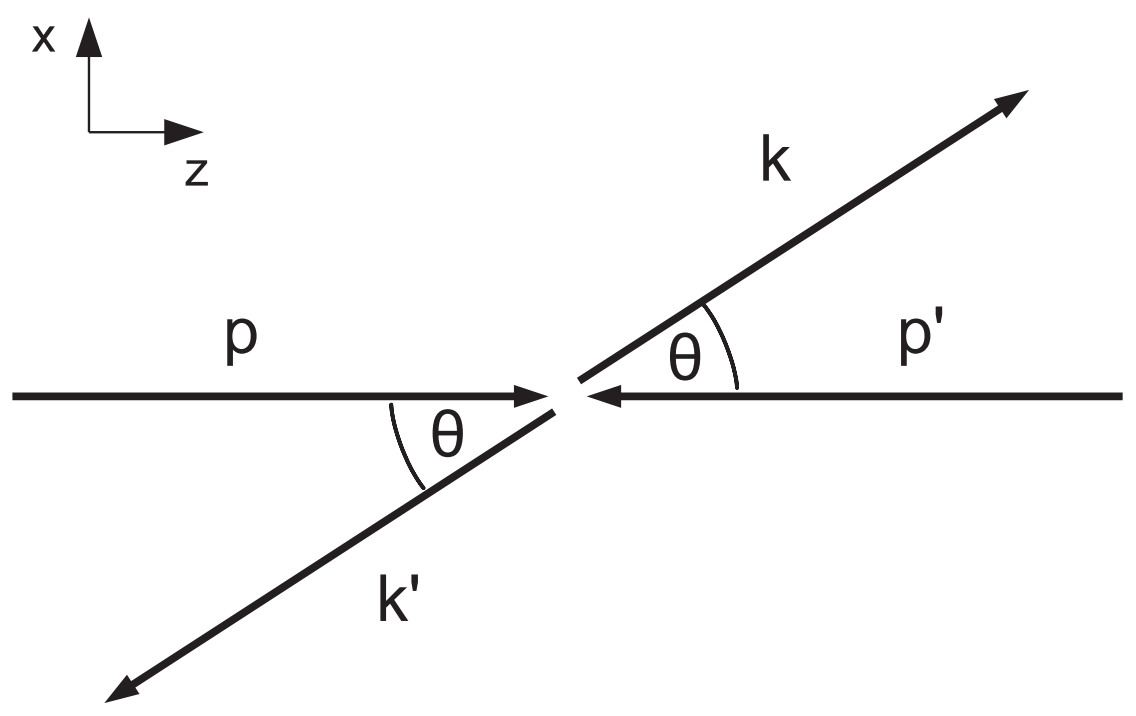

Figure 3.1: Two equal-mass particles in the centre-of-mass frame with fourmomenta $p$ and $p^{\prime}$ interact in a scattering event and emerge with four-momenta $k$ and $k^{\prime}$ at scattering angle $\theta$. The ingoing particles are taken to move along the $z$ axis, and the $x$ axis is chosen such that $\theta$ lies in the $\mathrm{x}-\mathrm{z}$ plane.

should each have magnitude of momentum $|\mathbf{p}|$. With these pieces of information established, a bit of trigonometry reveals that

$$
k=(E,|\mathbf{p}| \sin \theta, 0,|\mathbf{p}| \cos \theta) \quad \text { and } \quad k^{\prime}=(E,-|\mathbf{p}| \sin \theta, 0,-|\mathbf{p}| \cos \theta) .
$$

All of this is sufficient to convert Eq. (3.20) to the desired form. To that end, given that $E^{2}=|\mathbf{p}|^{2}+m_{\chi}{ }^{2}$ and that for four-momenta $a=\left(E_{a}, a_{x}, a_{y}, a_{z}\right)$ and $b=\left(E_{b}, b_{x}, b_{y}, b_{z}\right), a \cdot b=a^{\mu} g_{\mu \nu} b^{\nu}=E_{a} E_{b}-a_{x} b_{x}-a_{y} b_{y}-a_{z} b_{z}$, the following 
equations may be derived:

$$
\begin{gathered}
p \cdot p^{\prime}=2 E^{2}-m_{\chi}{ }^{2}, \\
p \cdot k=E^{2}(1-\cos \theta)+m_{\chi}{ }^{2} \cos \theta, \\
p \cdot k^{\prime}=E^{2}(1+\cos \theta)-m_{\chi}{ }^{2} \cos \theta, \\
p^{\prime} \cdot k=E^{2}(1+\cos \theta)-m_{\chi}{ }^{2} \cos \theta, \\
p^{\prime} \cdot k^{\prime}=E^{2}(1-\cos \theta)+m_{\chi}{ }^{2} \cos \theta, \\
k \cdot k^{\prime}=2 E^{2}-m_{\chi}{ }^{2}, \\
(p-k)^{2}=2\left(E^{2}-m_{\chi}{ }^{2}\right)(\cos \theta-1) \text { and } \\
\left(p-k^{\prime}\right)^{2}=-2\left(E^{2}-m_{\chi}{ }^{2}\right)(\cos \theta+1) .
\end{gathered}
$$

These equations may be used in tandem with Eq. (3.20) to give

$$
\begin{aligned}
\frac{1}{4} \sum_{\text {spins }}\left|\mathcal{M}_{\chi \chi \rightarrow \chi \chi}\right|^{2}= & 4 g^{4}\left\{\left[\frac{E^{2}(1-\cos \theta)+m_{\chi}^{2}(1+\cos \theta)}{2\left(E^{2}-m_{\chi}^{2}\right)(1-\cos \theta)+m_{\phi}^{2}}\right]^{2}\right. \\
& +\left[\frac{E^{2}(1+\cos \theta)+m_{\chi}^{2}(1-\cos \theta)}{2\left(E^{2}-m_{\chi}^{2}\right)(1+\cos \theta)+m_{\phi}^{2}}\right]^{2} \\
& \left.-\frac{\left(E^{2}-m_{\chi}^{2}\right)^{2}\left(\cos ^{2} \theta-1\right)+4 E^{2} m_{\chi}^{2}}{4\left(E^{2}-m_{\chi}^{2}\right)\left[m_{\phi}^{2}-\left(E^{2}-m_{\chi}^{2}\right)\left(\cos ^{2} \theta-1\right)\right]+m_{\phi}^{4}}\right\} .
\end{aligned}
$$

Note that this amplitude should be identical to the amplitude for the process $\bar{\chi} \bar{\chi} \rightarrow \bar{\chi} \bar{\chi}$ 


\section{Amplitude for $\bar{\chi} \chi \rightarrow \bar{\chi} \chi$}

The Feynman diagrams for this scattering process are shown below:
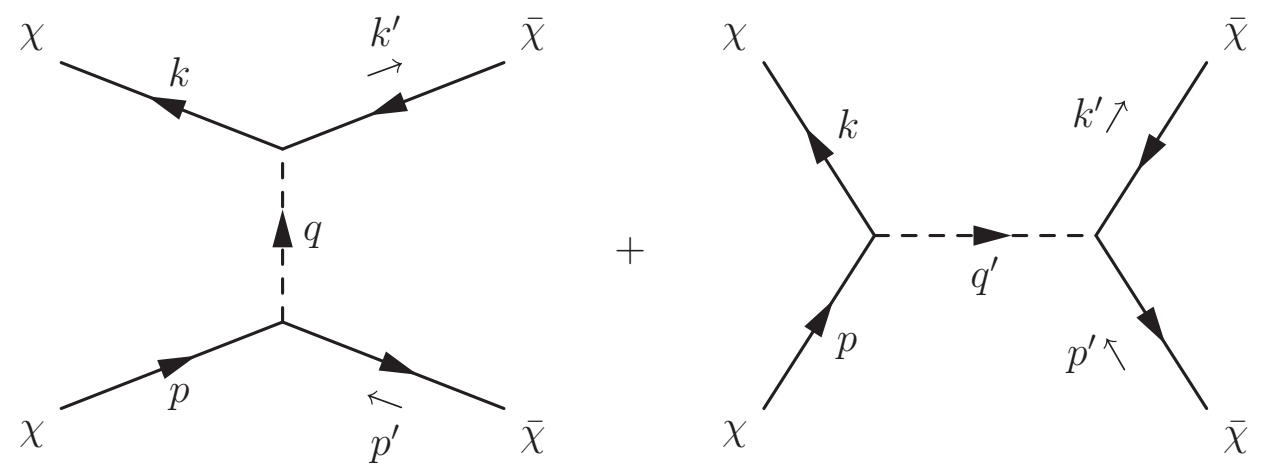

Applying the Feynman rules gives

$$
\begin{aligned}
i \mathcal{M}= & -\bar{v}\left(p^{\prime}\right)(-i g) u(p) \frac{i}{q^{2}-m_{\phi}^{2}} \bar{u}(k)(-i g) v\left(k^{\prime}\right) \\
& +\bar{v}\left(p^{\prime}\right)(-i g) v\left(k^{\prime}\right) \frac{i}{q^{\prime 2}-m_{\phi}^{2}} \bar{u}(k)(-i g) u(p),
\end{aligned}
$$

where $q=p+p^{\prime}$ and $q^{\prime}=p-k$. As in the $\chi \chi \rightarrow \chi \chi$ case, there is a relative minus sign between the terms.

Going through the very same logic as before - taking the absolute square of both sides, doing the trace calculations and applying the relevant kinematics to the interaction - leads to the following amplitude:

$$
\begin{aligned}
\frac{1}{4} \sum_{\text {spins }}\left|\mathcal{M}_{\bar{\chi} \chi \rightarrow \bar{\chi} \chi}\right|^{2}= & 4 g^{4}\left\{4\left(\frac{E^{2}-m_{\chi}^{2}}{4 E^{2}-m_{\phi}^{2}}\right)^{2}+\left[\frac{E^{2}(1-\cos \theta)+m_{\chi}^{2}(1+\cos \theta)}{\left(E^{2}-m_{\chi}^{2}\right)(1-\cos \theta)+m_{\phi}^{2}}\right]^{2}\right. \\
& \left.+2 \frac{\left(E^{2}-m_{\chi}^{2}\right)^{2}-\left(E^{4}-m_{\chi}^{4}\right) \cos \theta}{\left(4 E^{2}-m_{\phi}^{2}\right)\left[2\left(E^{2}-m_{\chi}^{2}\right)(1-\cos \theta)+m_{\phi}^{2}\right]}\right\} .
\end{aligned}
$$




\section{Amplitude for $\bar{\chi} \chi \rightarrow \phi \phi$}

This is the annihilation process. In allowing a three-way interaction scaled by $\tilde{g}$ between the $\phi$ particles, a third diagram called the s-channel process, so named because of its relation to the Mandelstam variables [25], is included. The three diagrams are shown below:

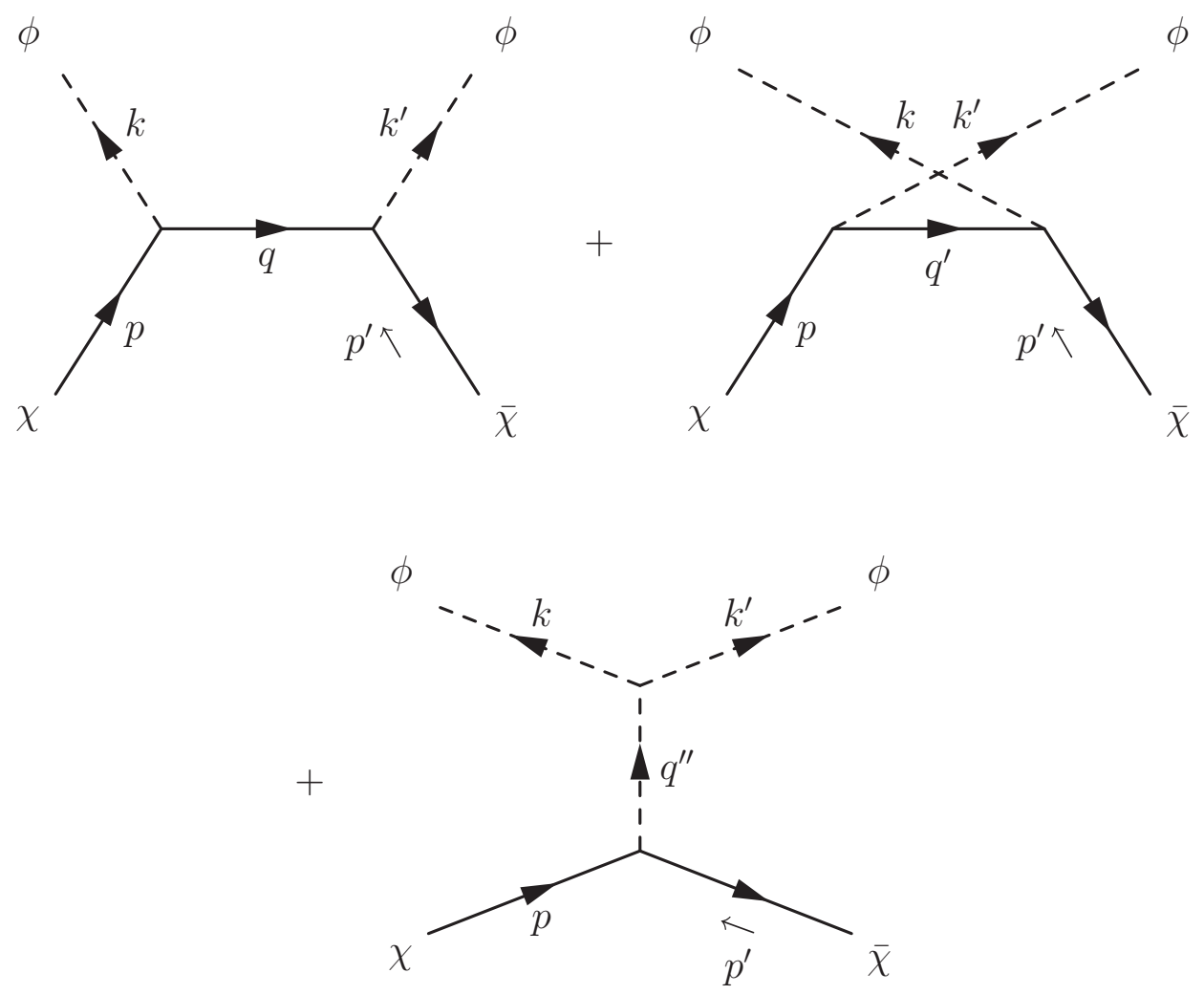


The diagrams indicate that

$$
\begin{aligned}
i \mathcal{M}= & \bar{v}\left(p^{\prime}\right)(-i g) \frac{i\left(\not 1+m_{\chi}\right)}{q^{2}-m_{\chi}^{2}}(-i g) u(p) \\
& +\bar{v}\left(p^{\prime}\right)(-i g) \frac{i\left(\phi^{\prime}+m_{\chi}\right)}{q^{\prime 2}-m_{\chi}^{2}}(-i g) u(p) \\
& +\bar{v}\left(p^{\prime}\right)(-i g) u(p) \frac{i}{q^{\prime \prime 2}-m_{\phi}^{2}}(-i \tilde{g}),
\end{aligned}
$$

where $q=p-k, q^{\prime}=p-k^{\prime}$ and $q^{\prime \prime}=p+p^{\prime}$. After going through the lengthy process of taking the absolute square and subsequently figuring out the kinematics, it may be shown that

$$
\begin{aligned}
& \frac{1}{4} \sum_{\text {spins }}\left|\mathcal{M}_{\bar{\chi} \chi \rightarrow \phi \phi}\right|^{2}= \\
& g^{4}\left[\frac{2 E^{4}-2 E^{2} m_{\phi}^{2}-8 m_{\chi}^{4}-8 m_{\chi}^{2} z \cos \theta+8 E^{2} m_{\chi}^{2}-2 z^{2} \cos ^{2} \theta}{\left(-2 E^{2}+2 z \cos \theta+m_{\phi}^{2}\right)^{2}}\right. \\
& +\frac{2 E^{4}-2 E^{2} m_{\phi}{ }^{2}-8 m_{\chi}{ }^{4}+8 m_{\chi}{ }^{2} z \cos \theta+8 E^{2} m_{\chi}{ }^{2}-2 z^{2} \cos ^{2} \theta}{\left(-2 E^{2}-2 z \cos \theta+m_{\phi}\right)^{2}} \\
& \left.+\frac{16 E^{2} m_{\chi}^{2}-16 m_{\chi}^{4}-4 E^{4}+4 E^{2} m_{\phi}^{2}+4 z^{2} \cos ^{2} \theta}{\left(-2 E^{2}+2 z \cos \theta+m_{\phi}^{2}\right)\left(-2 E^{2}-2 z \cos \theta+m_{\phi}^{2}\right)}\right] \\
& +g^{3} \tilde{g}\left[\frac{8 m_{\chi} E^{2}-8 m_{\chi}^{3}-4 m_{\chi} z \cos \theta}{\left(-2 E^{2}+2 z \cos \theta+m_{\phi}^{2}\right)\left(4 E^{2}-m_{\phi}^{2}\right)}\right. \\
& \left.+\frac{8 m_{\chi} E^{2}-8 m_{\chi}^{3}+4 m_{\chi} z \cos \theta}{\left(-2 E^{2}-2 z \cos \theta+m_{\phi}^{2}\right)\left(4 E^{2}-m_{\phi}^{2}\right)}\right]+g^{2} \tilde{g}^{2} \frac{2 E^{2}-2 m_{\chi}^{2}}{\left(4 E^{2}-m_{\phi}^{2}\right)^{2}},
\end{aligned}
$$

where

$$
z^{2}=\left(E^{2}-m_{\chi}^{2}\right)\left(E^{2}-m_{\phi}^{2}\right)
$$




\subsection{Scattering in the Non-Relativistic Limit}

With the amplitudes in hand, it is now possible to calculate the cross sections. Since the interactions between dark matter particles in Abell 3827 should be primarily scattering interactions, the annihilation cross section need not be calculated right away. The scattering cross section, on the other hand, is worth deriving here in order to obtain an interesting relationship between $m_{\chi}, m_{\phi}$ and $g$.

So far, every calculation has been exact, but comparison of Eq. (3.24) and Eq. (3.26) with Eq. (3.9) foreshadows a pair of rather cumbersome integrals. Fortunately, it should be possible to work in the non-relativistic limit because, as established in Ch. 2, dark matter needs to be quite cold in order to settle into galactic halos $[16,22]$. So long as $m_{\phi}$ is large compared to the momentum of the $\chi$, it makes sense to let the $\chi$ momentum simply go to zero because $E^{2}=|\mathbf{p}|^{2}+m_{\chi}{ }^{2}$, and if the dark matter velocity, $v$, goes to zero, so too does the momentum, $|\mathbf{p}|$. Working in the low-velocity limit, then, is equivalent to letting $E$ approach $m_{\chi}$ in Eq. (3.24) and Eq. (3.26). Upon investigation of those equations, it becomes clear that this approximation should only become problematic if $m_{\phi}$ is very small. (Later on in Ch. 4 , it will be shown that the $\phi$ is, in fact, quite light. The scattering cross section will therefore eventually need to be revisited.)

Working in the limit where $E \rightarrow m_{\chi}$ allows for a dramatic simplification 
of Eq. (3.24):

$$
\lim _{v \rightarrow 0} \frac{1}{4} \sum_{\text {spins }}\left|\mathcal{M}_{\chi \chi \rightarrow \chi \chi}\right|^{2}=\left(\frac{2 g m_{\chi}}{m_{\phi}}\right)^{4}
$$

Furthermore, investigation of Eq. (3.26) reveals that

$$
\lim _{v \rightarrow 0} \frac{1}{4} \sum_{\text {spins }}\left|\mathcal{M}_{\bar{\chi} \chi \rightarrow \bar{\chi} \chi}\right|^{2}=\left(\frac{2 g m_{\chi}}{m_{\phi}}\right)^{4}
$$

as well.

To write the cross section for the $\chi \chi \rightarrow \chi \chi$ process, one need simply take the amplitude, substitute it into Eq. (3.9) and integrate, yielding

$$
\sigma_{\chi \chi \rightarrow \chi \chi}=\int_{0}^{\pi} \int_{0}^{\pi} \frac{1}{2 E_{p} 2 E_{p^{\prime}}\left|v_{p}-v_{p^{\prime}}\right|} \frac{|\mathbf{k}|}{(2 \pi)^{2} 4 E_{\mathrm{CM}}}\left(\frac{2 g m_{\chi}}{m_{\phi}}\right)^{4} \sin \theta d \theta d \phi .
$$

Here the azimuthal angle is taken from 0 to $\pi$ instead of from 0 to $2 \pi$. This is to correct for the outgoing particles being identical; integrating over the full solid angle would count all the particle interactions twice, once for each of the two $\chi \mathrm{s}$ that emerge from a single scattering event.

To simplify the integral expression, recall that the kinematics were done in the centre-of-mass frame. It follows that $E_{p}=E_{p^{\prime}}=E, E_{C M}=2 E$, $\left|v_{p}-v_{p^{\prime}}\right|=2 v$ and $|\mathbf{k}|=m_{\chi} v$. Making these substitutions while setting $E=m_{\chi}$ and tidying everything up leads to

$$
\sigma_{\chi \chi \rightarrow \chi \chi}=\frac{m_{\chi}^{2} g^{4}}{8 \pi m_{\phi}^{4}}
$$


This cross section is the same for the $\bar{\chi} \bar{\chi} \rightarrow \bar{\chi} \bar{\chi}$ interaction, which is clear because the relevant Feynman diagrams for the $\bar{\chi} \bar{\chi} \rightarrow \bar{\chi} \bar{\chi}$ process are the same as the ones for the $\chi \chi \rightarrow \chi \chi$ process, just with the directional arrows running in opposite directions.

Because the amplitudes for the $\chi \chi \rightarrow \chi \chi$ and $\bar{\chi} \chi \rightarrow \bar{\chi} \chi$ processes are precisely identical in the non-relativistic limit, the cross sections will turn out identical as well, save for a factor of two introduced by running the azimuthal angle from 0 to $2 \pi$ for the $\bar{\chi} \chi \rightarrow \bar{\chi} \chi$ case. Thus,

$$
\sigma_{\bar{\chi} \chi \rightarrow \bar{\chi} \chi}=2 \sigma_{\chi \chi \rightarrow \chi \chi}=\frac{m_{\chi}^{2} g^{4}}{4 \pi m_{\phi}^{4}} .
$$

Assuming the $\chi$ and $\bar{\chi}$ particles exist in equal abundances, the total scattering cross section, $\sigma_{\text {scatt }}$, should be a simple average. Therefore,

$$
\sigma_{\text {scatt }}=\frac{\sigma_{\chi \chi \rightarrow \chi \chi}+\sigma_{\bar{\chi} \bar{\chi} \rightarrow \bar{\chi} \bar{\chi}}+\sigma_{\bar{\chi} \chi \rightarrow \bar{\chi} \chi}+\sigma_{\bar{\chi} \chi \rightarrow \chi \bar{\chi}}}{4},
$$

and, because $\sigma_{\chi \chi \rightarrow \chi \chi}=\sigma_{\bar{\chi} \bar{\chi} \rightarrow \bar{\chi} \bar{\chi}}$ and $\sigma_{\bar{\chi} \chi \rightarrow \bar{\chi} \chi}=\sigma_{\bar{\chi} \chi \rightarrow \chi \bar{\chi}}$,

$$
\sigma_{\text {scatt }}=\frac{\sigma_{\chi \chi \rightarrow \chi \chi}+\sigma_{\bar{\chi} \chi \rightarrow \bar{\chi} \chi}}{2}=\frac{3 m_{\chi}^{2} g^{4}}{16 \pi m_{\phi}^{4}},
$$

which may be rearranged to show that

$$
\frac{m_{\phi}}{g}=\left[\frac{3 m_{\chi}}{16 \pi\left(\frac{\sigma_{\text {scatt }}}{m_{\chi}}\right)}\right]^{\frac{1}{4}} .
$$




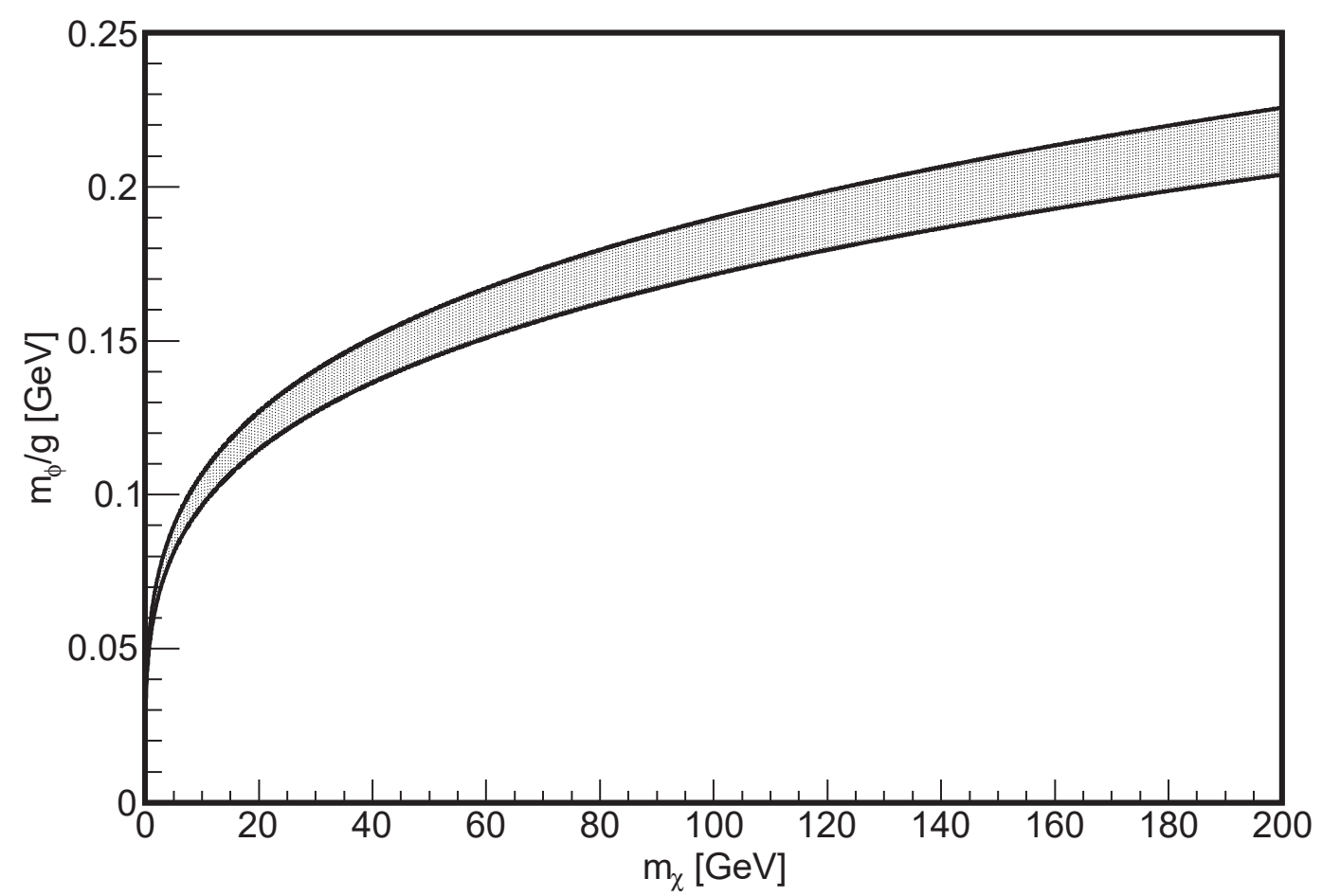

Figure 3.2: Constraints on $m_{\phi} / g$ based on observation of galaxy cluster Abell 3827 [1]. The allowed region is shaded and is defined by taking $\sigma_{\text {scatt }} / m_{\chi}$ to lie between 4600 and $6900 \mathrm{GeV}^{-3}$ [2]. We assume an equal abundance of $\chi$ and $\bar{\chi}$ particles.

Constraints on the quantity $\sigma_{\text {scatt }} / m_{\chi}$, which arose from observations of galaxy cluster Abell 3827, were provided in Eq. (2.4) [1, 2]. Converting the units (see App. A) leads to

$$
4600 \mathrm{GeV}^{-3} \lesssim \frac{\sigma_{\mathrm{scatt}}}{m_{\chi}} \lesssim 6900 \mathrm{GeV}^{-3}
$$

It is thus possible to plot $m_{\phi} / g$ as a function of $m_{\chi}$ (see Fig. 3.2) within a band of tolerance given by Eq. (3.37). Inspection of Fig. 3.2 reveals that $m_{\phi}$ remains relatively light for increasing $m_{\chi}$, assuming $g$ is not too large.

Further constraints may be placed upon this model, most notably those 
arising through consideration of the thermal history of dark matter over cosmological time scales, which is the subject of the next chapter. Indeed, the upcoming thermal history calculations act as an independent method to obtain a relationship between $m_{\chi}$ and $g$. As such, it will eventually be possible to eliminate $g$ from Eq. (3.36) entirely. 


\section{Chapter 4}

\section{Thermal Relics}

The present abundance of dark matter can be directly correlated with the annihilation cross section of its particles. As the universe cooled and expanded following the Big Bang, dark matter particles and antiparticles became increasingly dispersed, rarely running into one another. Eventually, a point was reached where annihilations essentially stopped, thereby leaving a so-called thermal relic abundance of dark matter particles. As a general rule, the higher the annihilation cross section, the lower the present abundance ought to be. The goal of this chapter is to use this information to eliminate $g$ from Eq. (3.36). 


\subsection{Freeze-Out}

In the very first moments of the Big Bang, the universe was in a state of approximate thermal equilibrium [15]. During the earliest epochs, the energy density of the universe was so high that particles were produced as often as they were destroyed. As such, the overall number of particles of a given particle species remained essentially fixed. As the universe continued to expand and cool, however, particle production became increasingly rare. Thus annihilation processes began to outweigh production processes, decreasing the number of particles available [26].

For particles that interact via the electromagnetic force or the strong nuclear force, particle annihilations would have continued until far fewer of them remained than are in existence today. Only the matter-antimatter asymmetry of baryons and charged leptons saved them from near extinction $[15,26]$. However, hypothetical particles that interact with a strength on the order of the weak scale (such as WIMPs) would have stopped annihilating with one another long ago due to their relatively feeble interaction strength. Thus any particular species of weakly-interacting particles should have, at some point, ceased tracking thermal equilibrium and should therefore have frozen out, leaving behind a thermal relic.

In order to calculate the relic abundance of a given dark matter candidate, it will be helpful to define a couple of parameters [26]. The first is the entropy 
density per comoving volume, $s$ :

$$
s=\frac{2 \pi^{2}}{45} d_{f s} T^{3}
$$

where $T$ is temperature and $d_{f s}$ is the effective number of degrees of freedom associated with the entropy density. The second is the expansion rate of the universe, $\mathrm{H}$, which is given during times of radiation dominance by

$$
H=\sqrt{\frac{8 \pi^{3} G}{90} d_{f} T^{4}},
$$

where $G$ is the gravitational constant and $d_{f}$ is the effective number of relativistic degrees of freedom associated with the energy density of the universe.

It will be elucidating here to go into a little more detail concerning $d_{f s}$ and $d_{f}$ (denoted by $g_{s}$ and $g_{\rho}$ in Ref. [26]). A plot of $d_{f}$ as a function of $T$ is given in Fig. 4.1. It is apparent from the plot that a hotter universe has more relativistic degrees of freedom associated with the energy density, a consequence of more particles being in thermal equilibrium with the universal heat bath. Fortunately, for all but the very lightest dark matter masses under consideration in this thesis $\left(m_{\chi}<10 \mathrm{MeV}\right), d_{f s}$ is equivalent to $d_{f}$ prior to freeze-out, and so any distinction between the two parameters need not be incorporated into the freeze-out model [26]. (In fact, by the end of Ch. 5 it will become clear that the allowed masses for the $\chi$ all fall between $10 \mathrm{MeV}$ and 


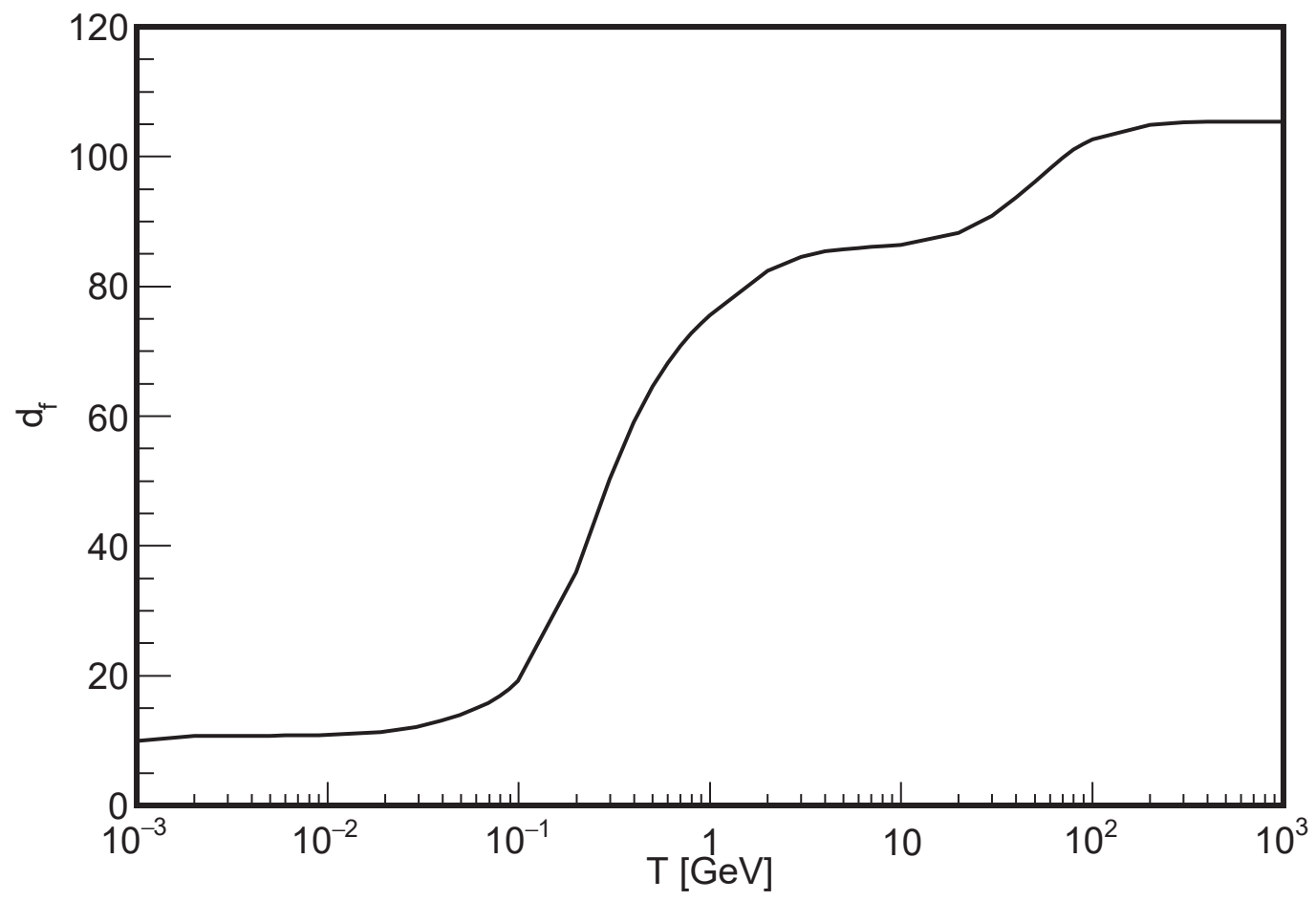

Figure 4.1: The effective number of relativistic degrees of freedom associated with the energy density of the universe, $d_{f}$, as a function of temperature, $T$. The functional form is taken from Appendix C of Ref. [27]. The plot clearly shows that when the universe is cooler, there are fewer degrees of freedom, which is a result of particles decoupling from the heat bath. From left to right, the first major rise in $d_{f}$ may be attributed to the quantum chromodynamic (QCD) transition and the second to the electroweak transition [26].

$10 \mathrm{TeV}$, the range of masses given in Ref. [26] that permits setting $d_{f s}=d_{f}$.)

With these quantities established, the relic abundance equation may be written as [26]

$$
\frac{d Y}{d x}=\frac{s\left\langle\sigma_{\mathrm{ann}} v_{\mathrm{rel}}\right\rangle}{H x}\left[1+\frac{1}{3} \frac{d\left(\ln d_{f s}\right)}{d(\ln T)}\right]\left(Y_{\mathrm{eq}}{ }^{2}-Y^{2}\right)
$$

where $Y=n / s$, and $n$ is the number density of dark matter particles per comoving volume, meaning that the effect of diminishing particle number density due to the expansion of the universe has no impact on $Y$. Further- 
more, $x=m / T$, where $m$ is the dark matter mass. As such, $x$ works rather like time because $x$ has inverse-temperature dependence, and the universe's temperature decreases monotonically throughout its evolution. The parameter $\left\langle\sigma_{\text {ann }} v_{\text {rel }}\right\rangle$ is the thermal average of the annihilation cross section multiplied by the relative velocity between dark matter particles, henceforth referred to simply as the thermally averaged annihilation cross section. Finally, $Y_{\mathrm{eq}}$ is the number density per unit entropy of dark matter particles per comoving volume when those particles are in thermal equilibrium with the universal heat bath. It is given by [26]

$$
Y_{\text {eq }}=\frac{45}{2 \pi^{4}}\left(\frac{\pi}{8}\right)^{1 / 2} \frac{d_{f \chi}}{d_{f s}} x^{3 / 2} e^{-x},
$$

where $d_{f \chi}$ is the effective number of degrees of freedom associated with the dark matter candidate.

As it happens, the derivative in the parentheses of Eq. (4.3) contributes very little, and so it may be neglected, leaving

$$
\frac{d Y}{d x}=\frac{s\left\langle\sigma_{\mathrm{ann}} v_{\mathrm{rel}}\right\rangle}{H x}\left(Y_{\mathrm{eq}}{ }^{2}-Y^{2}\right) .
$$

The goal is to solve this differential equation given some specified $\left\langle\sigma_{\text {ann }} v_{\text {rel }}\right\rangle$ and to see if the resulting $Y$ value at present is consistent with the observed dark matter relic abundance, thereby determining what value of $\left\langle\sigma_{\text {ann }} v_{\text {rel }}\right\rangle$ is 
correct [26]. As such, it will now be useful to find $\left\langle\sigma_{\text {ann }} v_{\text {rel }}\right\rangle$ in terms of the model parameters from Ch. 3 .

\subsection{Deriving $\left\langle\sigma_{\mathrm{ann}} v_{\text {rel }}\right\rangle$}

It was shown in Eq. (3.28) that the amplitude for the $\bar{\chi} \chi \rightarrow \phi \phi$ process is given by

$$
\begin{aligned}
& \frac{1}{4} \sum_{\text {spins }}\left|\mathcal{M}_{\bar{\chi} \chi \rightarrow \phi \phi}\right|^{2}= \\
& g^{4}\left[\frac{2 E^{4}-2 E^{2} m_{\phi}^{2}-8 m_{\chi}^{4}-8 m_{\chi}^{2} z \cos \theta+8 E^{2} m_{\chi}^{2}-2 z^{2} \cos ^{2} \theta}{\left(-2 E^{2}+2 z \cos \theta+m_{\phi}^{2}\right)^{2}}\right. \\
& +\frac{2 E^{4}-2 E^{2} m_{\phi}{ }^{2}-8 m_{\chi}{ }^{4}+8 m_{\chi}{ }^{2} z \cos \theta+8 E^{2} m_{\chi}{ }^{2}-2 z^{2} \cos ^{2} \theta}{\left(-2 E^{2}-2 z \cos \theta+m_{\phi}\right)^{2}} \\
& \left.+\frac{16 E^{2} m_{\chi}^{2}-16 m_{\chi}^{4}-4 E^{4}+4 E^{2} m_{\phi}^{2}+4 z^{2} \cos ^{2} \theta}{\left(-2 E^{2}+2 z \cos \theta+m_{\phi}^{2}\right)\left(-2 E^{2}-2 z \cos \theta+m_{\phi}^{2}\right)}\right] \\
& +g^{3} \tilde{g}\left[\frac{8 m_{\chi} E^{2}-8 m_{\chi}{ }^{3}-4 m_{\chi} z \cos \theta}{\left(-2 E^{2}+2 z \cos \theta+m_{\phi}^{2}\right)\left(4 E^{2}-m_{\phi}^{2}\right)}\right. \\
& \left.+\frac{8 m_{\chi} E^{2}-8 m_{\chi}^{3}+4 m_{\chi} z \cos \theta}{\left(-2 E^{2}-2 z \cos \theta+m_{\phi}^{2}\right)\left(4 E^{2}-m_{\phi}^{2}\right)}\right]+g^{2} \tilde{g}^{2} \frac{2 E^{2}-2 m_{\chi}{ }^{2}}{\left(4 E^{2}-m_{\phi^{2}}\right)^{2}},
\end{aligned}
$$

where

$$
z^{2}=\left(E^{2}-m_{\chi}^{2}\right)\left(E^{2}-m_{\phi}^{2}\right)
$$

In order to find the corresponding cross section, the same logic as in Sec. 3.3 may be applied. Unfortunately, working in the low-velocity limit is out of the 
question, as

$$
\lim _{v_{\mathrm{rel}} \rightarrow 0} \frac{1}{4} \sum_{\text {spins }}\left|\mathcal{M}_{\bar{\chi} \chi \rightarrow \phi \phi}\right|^{2}=0
$$

so the first step will be to integrate over $\cos \theta$.

By defining the momenta as in Eq. (3.27) and noting the form of Eq. (3.31) in Sec. 3.3, it may be shown that

$\sigma_{\bar{\chi} \chi \rightarrow \phi \phi}=\int_{0}^{\pi} \int_{0}^{\pi} \frac{1}{2 E_{p} 2 E_{p^{\prime}}\left|v_{p}-v_{p^{\prime}}\right|} \frac{|\mathbf{k}|}{(2 \pi)^{2} 4 E_{\mathrm{CM}}}\left(\frac{1}{4} \sum_{\mathrm{spins}}\left|\mathcal{M}_{\bar{\chi} \chi \rightarrow \phi \phi}\right|^{2}\right) \sin \theta d \theta d \phi$,

where $E_{p}=E_{p^{\prime}}=E, E_{C M}=2 E,\left|v_{p}-v_{p^{\prime}}\right|=2 v=v_{\text {rel }},|\mathbf{p}|=m_{\chi} v_{\text {rel }} / 2=$ $\sqrt{E^{2}-m_{\chi}^{2}}$ and $|\mathbf{k}|=\sqrt{E^{2}-m_{\phi}^{2}}$. Simplifying leads to the following equation:

$$
\sigma_{\mathrm{ann}}=\sigma_{\bar{\chi} \chi \rightarrow \phi \phi}=\frac{m_{\chi}\left(E^{2}-m_{\phi}^{2}\right)}{256 \pi E^{3} z} \int_{-1}^{1} \frac{1}{4} \sum_{\text {spins }}\left|\mathcal{M}_{\bar{\chi} \chi \rightarrow \phi \phi}\right|^{2} d \cos \theta
$$

The integral in Eq. (4.10) is difficult and tedious but ultimately solvable 
through the use of partial fractions. The result is given by

$$
\begin{aligned}
& \sigma_{\text {ann }}=\frac{m_{\chi} g^{4}}{256 \pi E^{3}\left(E^{2}-m_{\chi}^{2}\right)}\left\{-4 z+\left(-3 E^{2}+\frac{3}{2} m_{\phi}^{2}-4 m_{\chi}^{2}\right.\right. \\
& \left.+\frac{8 E^{2} m_{\chi}^{2}-8 m_{\chi}^{4}-2 E^{4}+2 E^{2} m_{\phi}^{2}}{-2 E^{2}+m_{\phi}^{2}}\right) \ln \left|\frac{-2 E^{2}+m_{\phi}^{2}+2 z}{-2 E^{2}+m_{\phi}^{2}-2 z}\right| \\
& \left.+\left(m_{\phi}^{2}-4 m_{\chi}^{2}\right)\left(\frac{1}{2} m_{\phi}^{2}-2 m_{\chi}^{2}\right)\left(\frac{1}{-2 E^{2}+m_{\phi}^{2}+2 z}-\frac{1}{-2 E^{2}+m_{\phi}^{2}-2 z}\right)\right\} \\
& +\frac{m_{\chi}}{128 \pi E^{3}\left(E^{2}-m_{\chi}^{2}\right)}\left[-g^{3} \tilde{g} \frac{4 z m_{\chi}}{4 E^{2}-m_{\phi}^{2}}\right. \\
& \left.+g^{3} \tilde{g} \frac{2 m_{\chi} E^{2}-4 m_{\chi}^{3}+m_{\chi} m_{\phi}^{2}}{4 E^{2}-m_{\phi}^{2}} \ln \left|\frac{-2 E^{2}+m_{\phi}^{2}+2 z}{-2 E^{2}+m_{\phi}^{2}-2 z}\right|+g^{2} \tilde{g}^{2} \frac{z\left(2 E^{2}-2 m_{\chi}^{2}\right)}{\left(4 E^{2}-m_{\phi}^{2}\right)^{2}}\right] .
\end{aligned}
$$

Clearly, Eq. (4.11) is far more unwieldy than, for example, Eq. (3.35). In order to rein in Eq. (4.11), it will be necessary to make some simplifying assumptions. First, it has already been noted that unless the coupling factor $g$ is very large, $m_{\phi}$ must be very small compared to $m_{\chi}$ (see Fig. 3.2). As will be seen later, $g$ cannot be overly large, so setting $m_{\phi}=0$ in the annihilation cross section should be acceptable. Thus Eq. (4.11) — multiplied by $v_{\text {rel }}$ in keeping 
with the goal of finding $\left\langle\sigma_{\text {ann }} v_{\text {rel }}\right\rangle$-reduces to

$$
\begin{aligned}
\sigma_{\text {ann }} v_{\text {rel }}= & \frac{g^{4}}{128 \pi E^{3}|\mathbf{p}|}\left\{-4 z+\left(-2 E^{2}-8 m_{\chi}^{2}+4 \frac{m_{\chi}{ }^{4}}{E^{2}}\right) \ln \left|\frac{E^{2}-z}{E^{2}+z}\right|\right. \\
& \left.+4 m_{\chi}^{4}\left(\frac{1}{E^{2}+z}-\frac{1}{E^{2}-z}\right)\right\}+\frac{1}{64 \pi E^{3}|\mathbf{p}|}\{ \\
& \left.g^{3} \tilde{g} m_{\chi}\left(\frac{1}{2}-\frac{m_{\chi}^{2}}{E^{2}}\right) \ln \left|\frac{E^{2}-z}{E^{2}+z}\right|-g^{3} \tilde{g} \frac{z m_{\chi}}{E^{2}}+g^{2} \tilde{g}^{2} \frac{z}{8 E^{2}}\left(1-\frac{m_{\chi}^{2}}{E^{2}}\right)\right\} .
\end{aligned}
$$

The next step is to take Eq. (4.12) and write it to leading order in $v_{\text {rel }}$. To do so, it will be useful to employ relativistic expressions for the energies and momenta. Thus $E=\gamma m_{\chi}$ and $|\mathbf{p}|=\gamma m_{\chi} v_{\text {rel }} / 2$, where $\gamma$ is the Lorentz factor defined by

$$
\gamma=\frac{1}{\sqrt{1-\frac{v_{\mathrm{rel}}^{2}}{4}}} .
$$

It follows that $z=\gamma^{2} m_{\chi}{ }^{2} v_{\text {rel }} / 2$. Making use of these expressions, rather than their non-relativistic counterparts, is not strictly necessary; however, doing so interestingly leads to a much simpler derivation of Eq. (4.12) to leading order in $v_{\text {rel }}$.

Proceeding is best accomplished by first breaking Eq. (4.12) into several 
parts:

$$
\begin{aligned}
& P_{1}=\frac{g^{4}}{128 \pi E^{3}|\mathbf{p}|}, \\
& P_{2}=-4 z \\
& P_{3}=-2 E^{2}-8 m_{\chi}^{2}+4 \frac{m_{\chi}^{4}}{E^{2}}, \\
& P_{4}=\ln \left|\frac{E^{2}-z}{E^{2}+z}\right|, \\
& P_{5}=4 m_{\chi}^{4}\left(\frac{1}{E^{2}+z}-\frac{1}{E^{2}-z}\right), \\
& P_{6}=\frac{1}{64 \pi E^{3}|\mathbf{p}|}, \\
& P_{7}=g^{3} \tilde{g} m_{\chi}\left(\frac{1}{2}-\frac{m_{\chi}^{2}}{E^{2}}\right) \\
& P_{8}=-g^{3} \tilde{g} \frac{z m_{\chi}}{E^{2}} \text { and } \\
& P_{9}=g^{2} \tilde{g}^{2} \frac{z}{8 E^{2}}\left(1-\frac{m_{\chi}^{2}}{E^{2}}\right) .
\end{aligned}
$$

These parts should subsequently be written in terms of $v_{\text {rel }}$ and $\gamma$. Applying the aforementioned relativistic expressions for energy and momentum, it may 
be shown that

$$
\begin{aligned}
& P_{1}=\frac{g^{4}}{64 \pi m_{\chi}{ }^{4}} \frac{1}{\gamma^{4}} \frac{1}{v_{\text {rel }}}, \\
& P_{2}=-2 \gamma^{2} m_{\chi}{ }^{2} v_{\text {rel }}, \\
& P_{3}=m_{\chi}^{2}\left(-2 \gamma^{2}-8+\frac{4}{\gamma^{2}}\right), \\
& P_{4}=\ln \left|\frac{1-v_{\text {rel }} / 2}{1+v_{\text {rel }} / 2}\right|, \\
& P_{5}=-4 v_{\text {rel }}, \\
& P_{6}=\frac{1}{32 \pi m_{\chi}^{4}} \frac{1}{\gamma^{4}} \frac{1}{v_{\text {rel }}}, \\
& P_{7}=g^{3} \tilde{g} m_{\chi}\left(\frac{1}{2}-\frac{1}{\gamma^{2}}\right), \\
& P_{8}=-g^{3} \tilde{g} \frac{m_{\chi} v_{\text {rel }}}{2} \text { and } \\
& P_{9}=g^{2} \tilde{g}^{2} \frac{1}{16} v_{\text {rel }}\left(1-\frac{1}{\gamma^{2}}\right) .
\end{aligned}
$$

Next, if Eq. (4.12) is written as a sum of the terms $t_{1}=P_{1} P_{2}, t_{2}=P_{1} P_{3} P_{4}$, 


$$
\begin{aligned}
t_{3}=P_{1} P_{5}, t_{4} & =P_{6} P_{7} P_{4}, t_{5}=P_{6} P_{8} \text { and } t_{6}=P_{6} P_{9}, \text { then } \\
t_{1} & =-\frac{g^{4}}{32 \pi m_{\chi}^{2}} \frac{1}{\gamma^{2}}, \\
t_{2} & =\frac{g^{4}}{32 \pi m_{\chi}^{2}}\left(-\frac{1}{\gamma^{2}}-\frac{4}{\gamma^{2}}+\frac{2}{\gamma^{6}}\right)\left(\frac{1}{v_{\text {rel }}}\right)\left(\ln \left|\frac{1-v_{\text {rel }} / 2}{1+v_{\text {rel }} / 2}\right|\right) \\
t_{3} & =\frac{-2 g^{4}}{32 \pi m_{\chi}^{2}} \frac{1}{\gamma^{4}}, \\
t_{4} & =\frac{g^{3} \tilde{g}}{32 \pi m_{\chi}^{3}}\left(\frac{1}{2 \gamma^{4}}-\frac{1}{\gamma^{6}}\right)\left(\frac{1}{v_{\text {rel }}}\right)\left(\ln \left|\frac{1-v_{\text {rel }} / 2}{1+v_{\text {rel }} / 2}\right|\right) \\
t_{5} & =\frac{g^{3} \tilde{g}}{32 \pi m_{\chi}^{3}}\left(\frac{-1}{2 \gamma^{4}}\right) \text { and } \\
t_{6} & =\frac{g^{2} \tilde{g}^{2}}{512 \pi m_{\chi}^{4}}\left(\frac{1}{\gamma^{4}}-\frac{1}{\gamma^{6}}\right) .
\end{aligned}
$$

Now,

$$
\frac{1}{\gamma^{2}}=1-\frac{v_{\mathrm{rel}}^{2}}{4}
$$

and to quadratic order in $v_{\text {rel }}$,

$$
\frac{1}{\gamma^{4}}=1-\frac{v_{\text {rel }^{2}}^{2}}{2} \text { and } \frac{1}{\gamma^{6}}=1-\frac{3 v_{\text {rel }^{2}}}{4}
$$

In addition, performing a Taylor series expansion on the logarithmic term yields

$$
\ln \left|\frac{1-v_{\text {rel }} / 2}{1+v_{\text {rel }} / 2}\right|=-v_{\text {rel }}-\frac{v_{\text {rel }}^{3}}{12}
$$

to cubic order. Adding all the $t$ terms together while simultaneously making the above substitutions gives a $\sigma_{\text {ann }} v_{\text {rel }}$ accurate to second order in $v_{\text {rel }}$. The 
constant terms cancel, which is consistent with the fact that the amplitude goes to zero in the low-velocity limit. This leaves

$$
\sigma_{\mathrm{ann}} v_{\mathrm{rel}}=\frac{3 g^{4} v_{\mathrm{rel}^{2}}}{128 \pi m_{\chi}^{2}}-\frac{5 g^{3} \tilde{g} v_{\mathrm{rel}^{2}}^{2}}{768 \pi m_{\chi}^{3}}+\frac{g^{2} \tilde{g}^{2} v_{\mathrm{rel}}^{2}}{2048 \pi m_{\chi}{ }^{4}}
$$

The annihilation cross section has been vastly simplified, yet there is one more step worth pursuing. For simplicity, we set the coupling $\tilde{g}$ to zero. Given that the mass of the $\phi$ is so very low, it stands to reason that the s-channel diagram should be highly suppressed anyway $[20,25]$. To wit, if the s-channel process is to occur, then the virtual $\phi$ must carry the energy of the annihilating dark matter particles, an amount of energy greatly exceeding that which a real $\phi$ could carry. As a result, the virtual $\phi$ would have to be far off its mass shell. This effect may be seen quantitatively by investigating the form of the Feynman rule for the scalar propagator in Sec. 3.1; clearly, the greater the difference between $m_{\phi}$ and $q$, the larger the denominator in the Feynman rule will become. This effect ultimately leads to a suppression of the process, so it will be sufficient here to set $\tilde{g}=0$ and ignore this contribution. Thus the final form of the annihilation cross section is given by

$$
\sigma_{\mathrm{ann}} v_{\mathrm{rel}}=\frac{3 g^{4} v_{\mathrm{rel}}^{2}}{128 \pi m_{\chi}^{2}}
$$


To make Eq. (4.18) more compact, the variable $\alpha$ is defined such that

$$
\alpha=\frac{3 g^{4}}{128 \pi m_{\chi}^{2}} .
$$

Then

$$
\sigma_{\text {ann }} v_{\text {rel }}=\alpha v_{\text {rel }}^{2}
$$

With Eq. (4.20) so succinct, the machinery of statistical mechanics may at last be used to derive the thermal average of $\sigma_{\mathrm{ann}} v_{\text {rel }}$.

The probability, $P_{i}$, of state $i$ arising with energy $E_{i}$ from amongst a discrete set of states is given by the canonical probability distribution,

$$
P_{i}=\frac{e^{-E_{i} / k_{\mathrm{B}} T}}{\sum_{j} e^{-E_{j} / k_{\mathrm{B}} T}}
$$

where $j$ runs over all states, $k_{\mathrm{B}}$, the Boltzmann constant, is equal to 1 in natural units and the numerator is called the Boltzmann factor [28]. To find the thermal average of the annihilation cross section, it is first necessary to specify the energy of a particular state. The energy is, of course, the centreof-mass energy given by $E_{\mathrm{CM}}=2 E=2\left(m_{\chi} v^{2} / 2\right)=m_{\chi} v_{\text {rel }}^{2} / 4$. Now, because $v_{\text {rel }}$ is a continuous variable, an integral version of Eq. (4.21) is required. To this end, integrating the Boltzmann factor over all possible $\mathbf{v}_{\text {rel }}$ leads to a 
probability density function [29]:

$$
1=\int_{0}^{2 \pi} \int_{0}^{\pi} \int_{0}^{\infty} N e^{-m_{\chi} v_{\text {rel }}^{2} / 4 T} v_{\text {rel }}^{2} \sin \theta d v_{\text {rel }} d \theta d \phi,
$$

where $N$ is a normalization constant. Performing the solid angle integration leads to

$$
1=4 \pi N \int_{0}^{\infty} e^{-m_{\chi} v_{\mathrm{rel}}^{2} / 4 T} v_{\mathrm{rel}}^{2} d v_{\mathrm{rel}}
$$

which contains a Gaussian integral. In general, the solution for a Gaussian integral with even powers of $x$ is given by [30]

$$
\int_{0}^{\infty} x^{2 n} e^{-x^{2} / a^{2}} d x=\sqrt{\pi} \frac{(2 n) !}{n !}\left(\frac{a}{2}\right)^{2 n+1}
$$

where $n$ is an integer. Taking this general solution and applying it to the case of Eq. (4.23) yields

$$
N=\left(\frac{m_{\chi}}{4 \pi T}\right)^{\frac{3}{2}}
$$

Taking note of Eq. (4.20), Eq. (4.22) and Eq. (4.25), the thermally averaged annihilation cross section is

$$
\left\langle\sigma_{\text {ann }} v_{\text {rel }}\right\rangle=\int_{0}^{2 \pi} \int_{0}^{\pi} \int_{0}^{\infty} \alpha v_{\text {rel }}^{2}\left(\frac{m_{\chi}}{4 \pi T}\right)^{\frac{3}{2}} e^{-m_{\chi} v_{\text {rel }}{ }^{2} / 4 T} v_{\text {rel }}^{2} \sin \theta d v_{\text {rel }} d \theta d \phi,
$$

and, again making use of Eq. (4.24) in order to perform the integration, it 
follows that

$$
\left\langle\sigma_{\text {ann }} v_{\text {rel }}\right\rangle=6 \alpha \frac{T}{m_{\chi}}
$$

\subsection{Determining $\alpha$}

As stated earlier, the goal of this chapter is to eliminate $g$ from Eq. (3.36). With Eq. (4.19) and Eq. (4.27) established, it is clear that that goal will soon be accomplished. All that is left now is to determine $\alpha$ as a function of $m_{\chi}$, the prescription for which was detailed in Sec. 4.1. To reiterate, Eq. (4.5) must be solved for a given combination of $\left\langle\sigma_{\text {ann }} v_{\text {rel }}\right\rangle$ and $m_{\chi}$; then the resulting $Y$ value at late times is compared against current dark matter relic abundance observations. Thanks to Eq. (4.27), the problem of finding the $\left\langle\sigma_{\text {ann }} v_{\text {rel }}\right\rangle$ that corresponds to a given $m_{\chi}$ immediately reduces to the problem of finding the $\alpha$ value that does so. To be precise, substitution of Eq. (4.1), Eq. (4.2) and Eq. (4.27) into Eq. (4.5) gives

$$
\frac{d Y}{d x}=\left(6 \alpha d_{f s} \sqrt{\frac{\pi}{45 G d_{f}}}\right)\left(Y_{\mathrm{eq}}^{2}-Y^{2}\right) \frac{m_{\chi}}{x^{3}},
$$

which has parameters $\alpha$ and $m_{\chi}$ and is entirely a function of $x$, as is clear upon investigation of Eq. (4.4) and Fig. 4.1.

In a majority of models, $\sigma_{\text {ann }} v_{\text {rel }}$ works out to a constant to leading order in $v_{\text {rel }}$. In such cases, the annihilation is said to be an s-wave annihilation, 
referring to the orbital angular momentum of the initial state. Alternatively, annihilation cross sections that are proportional to $v_{\text {rel }}^{2}$ at leading order, as is the case for our model, are said to be p-wave annihilations [31]. In solving Eq. (4.28) - using, for example, the fourth-order Runge-Kutta technique (see App. B) - it is generally less computationally taxing to address the p-wave case than the s-wave case. In either instance, however, the process is identical. One starts by assuming the dark matter particles are in thermal equilibrium $\left(Y=Y_{\text {eq }}\right)$ at $x \gtrsim 1$, and then $Y$ is generated for increasing $x$. This is continued until long after freeze-out occurs in order to compare $Y$ with observation [26].

To make the comparison, a numerical value for $Y$ at present needs to be obtained. Let a zero subscript denote the present-day value of a given variable. Then $[26,32,33]$

$$
Y_{0}=\frac{135}{16 \pi^{3}} \frac{\Omega_{\mathrm{CDM}} H_{0}{ }^{2}}{G m_{\chi} d_{f s} T_{0}^{3}},
$$

where $\Omega_{\mathrm{CDM}}$ is the cold dark matter density parameter. It is important to note here that at present time $d_{f s}=3.91$ [33], which is different from the value of $d_{f}$ during the current stage of the universe's evolution. Using the currently accepted values for all the parameters (see Table 1 ) and taking $m_{\chi}=100 \mathrm{GeV}$ for a sample point yields

$$
Y_{0}\left(m_{\chi}=100 \mathrm{GeV}\right)=4.31 \times 10^{-12}
$$

With Eq. (4.30) now identified as the condition for success, a solution to 


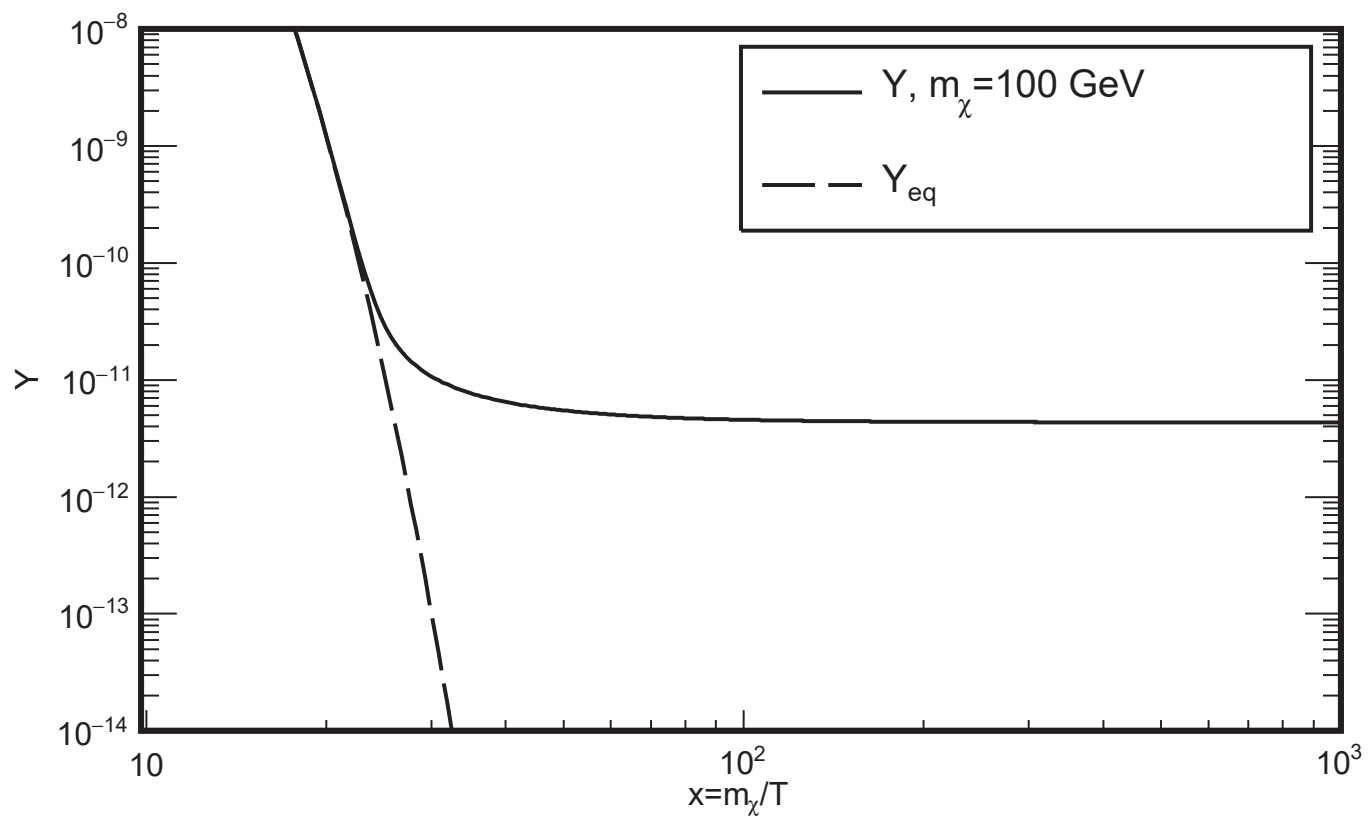

Figure 4.2: A depiction of thermal freeze-out when the dark matter mass is taken to be $100 \mathrm{GeV}$ and $d_{f \chi}=4$. Arriving at the correct present relic abundance as given in Eq. (4.30) requires that $\alpha=1.41 \times 10^{-8} \mathrm{GeV}^{-2}$.

Eq. (4.28) may be found. A plot of the solution is given in Fig. 4.2. The plot depicts the freeze-out process for the sample case where $m_{\chi}=100 \mathrm{GeV}$. The effective number of degrees of freedom for our dark matter candidate, $d_{f \chi}$, is set to 4 because there is one Dirac fermion (manifesting as $\chi$ and $\bar{\chi}$ ) with two spins. It is instructive to note how the dark matter relic density curve (solid) tracks the thermal equilibrium curve (dashed) until freeze-out occurs, when the line subsequently veers away and runs flat. The overall effect is the same as in the s-wave case, save that the p-wave curve tracks equilibrium a little longer before taking a sharper turn at freeze-out.

The input $\alpha=1.41 \times 10^{-8} \mathrm{GeV}^{-2}$ was chosen to arrive at the solution in Fig. 4.2. In this very same manner, $Y$ may be matched with $Y_{0}$ for each value of $m_{\chi}$, ultimately leading to the ability to find $\alpha$ for all $m_{\chi}$. In principle this 


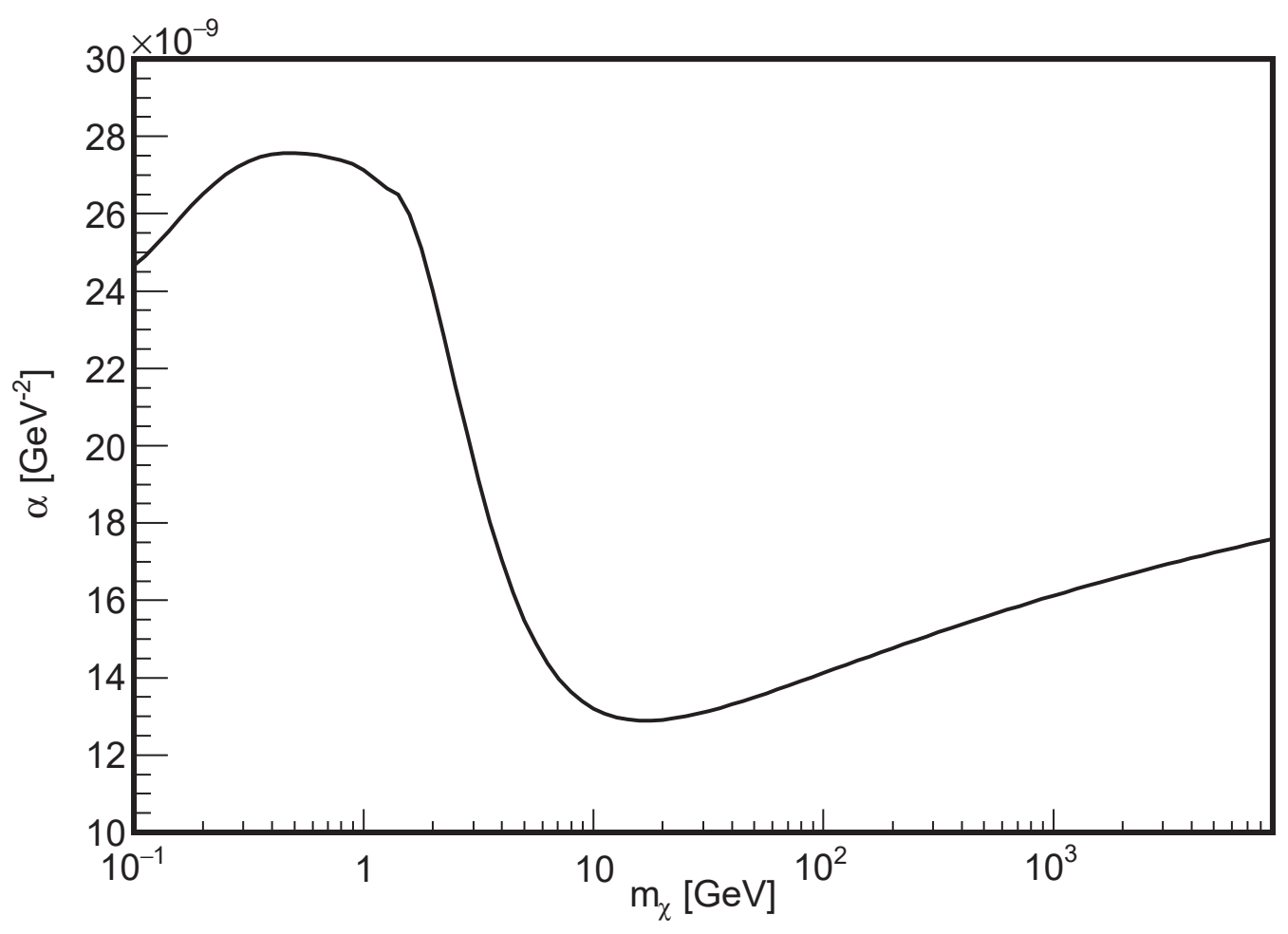

Figure 4.3: A plot of the parameter $\alpha$ from Eq. (4.27) as a function of the dark matter mass. In this case, $d_{f \chi}=4$, which matches our model. The data used to generate this plot was taken from Ref. [27].

process could be accomplished using the fourth-order Runge-Kutta technique just as in Fig. 4.2, but that is a very time-consuming proposition; for, in order to perform the integration, the step size needs to be extremely small (see App. B). Better is to use the so-called Möbius scheme to solve the differential equation, which, though more complicated, is designed specifically to deal with differential equations such as Eq. (4.28). See Ref. [27] for more information on the Möbius scheme. Here it will suffice to demonstrate the end result of its use, which is shown in Fig. 4.3. 


\subsection{A Relationship Between $m_{\chi}$ and $m_{\phi}$}

With $\alpha$ determined as a function of $m_{\chi}$, it is now possible to write $g$ as a function of $m_{\chi}$ through rearranging Eq. (4.19) and isolating $g$ :

$$
g=\left(\frac{128 \pi m_{\chi}{ }^{2} \alpha}{3}\right)^{\frac{1}{4}}
$$

Before relating Eq. (4.31) to Eq. (3.36), however, one important point should be established. Earlier it was stated that $g$ cannot be a very large number. That statement will now be made quantitative by way of setting an upper bound on $g$.

To set the limit, it is important to first consider the decay process $\phi \rightarrow \bar{\chi} \chi$ [34]:

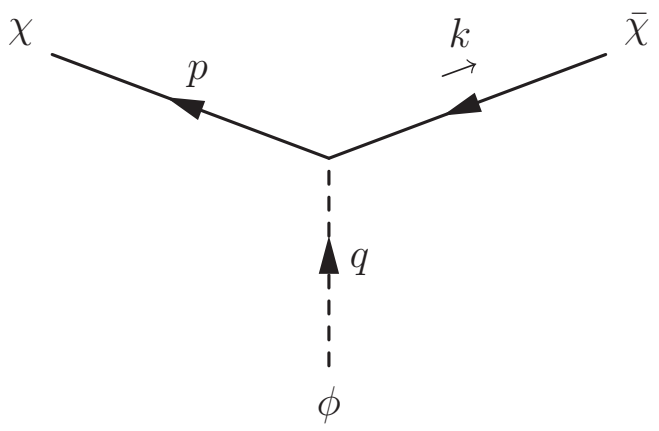

Although $2 m_{\chi}$ is greater than $m_{\phi}$, rendering the decay nonphysical, a theoretical upper bound on $g$ may still be established. From the Feynman diagram, then,

$$
i \mathcal{M}=\bar{u}(p)(-i g) v(k)
$$


and working through the kinematics leads to

$$
\sum_{\text {spins }}|\mathcal{M}|^{2}=2 g^{2}\left(m_{\phi}^{2}-4 m_{\chi}^{2}\right)
$$

At this point, it is the decay width, $\Gamma$, rather than the cross section, that is of interest. The decay width is the probability per unit time that a given particle will decay, and the general formula for the decay width of a scalar boson $A$ of mass $m_{A}$ that decays to a pair of non-identical fermions, each with momentum p, is given by [20]

$$
\Gamma=\frac{|\mathbf{p}|}{8 \pi m_{A}^{2}} \sum_{\text {spins }}|\mathcal{M}|^{2}
$$

Using this formula in tandem with Eq. (4.33) and recalling that $\mathbf{p}^{2}=E^{2}-m_{\chi}{ }^{2}$, while also noting that $E=m_{\phi}$ in this case, leads to

$$
\Gamma_{\phi \rightarrow \bar{\chi} \chi}=\frac{g^{2}}{\pi m_{\phi}^{2}}\left(\frac{1}{4} m_{\phi}^{2}-m_{\chi}^{2}\right)^{\frac{3}{2}} .
$$

The Feynman diagram shown for this decay process should capture the amplitude of the decay to high precision, but it is only a first order approximation [25]. There are higher-order Feynman diagrams with loops that could also be taken into account if more precision were required. To keep it so that higher-order Feynman diagrams do not contribute more to the decay width than the simpler Feynman diagrams - to keep the calculation perturbative - a 
boundary should be imposed on the quantity $\Gamma_{\phi \rightarrow \bar{\chi} \chi} / m_{\phi}$. In keeping with Ref. [34], that boundary is given by

$$
\frac{\Gamma_{\phi \rightarrow \bar{\chi} \chi}}{m_{\phi}}<\frac{1}{2},
$$

which, taking note of Eq. (4.35), implies that

$$
\frac{g^{2}}{\pi m_{\phi}{ }^{3}}\left(\frac{1}{4} m_{\phi}{ }^{2}-m_{\chi}{ }^{2}\right)^{\frac{3}{2}}<\frac{1}{2}
$$

or

$$
g^{2}<\frac{\pi m_{\phi}^{3}}{2\left(\frac{1}{4} m_{\phi}{ }^{2}-m_{\chi}{ }^{2}\right)^{3 / 2}} .
$$

Maximizing the right-hand side of Eq. (4.38) allows for the loosest possible bound, so $m_{\chi}$ is taken to be zero. Given that $g$ is positive by definition, it follows that

$$
g<2 \sqrt{\pi} .
$$

The limit on $g$ now established, Eq. (4.31) may be combined with Eq. (4.39) in order to describe the relationship between $g$ and $m_{\chi}$. A plot of this relationship is provided in Fig. 4.4. Note the horizontal dashed line set at $2 \sqrt{\pi}$; this limit placed on $g$ also places a limit on $m_{\chi}$. Specifically,

$$
m_{\chi}<8200 \mathrm{GeV}
$$




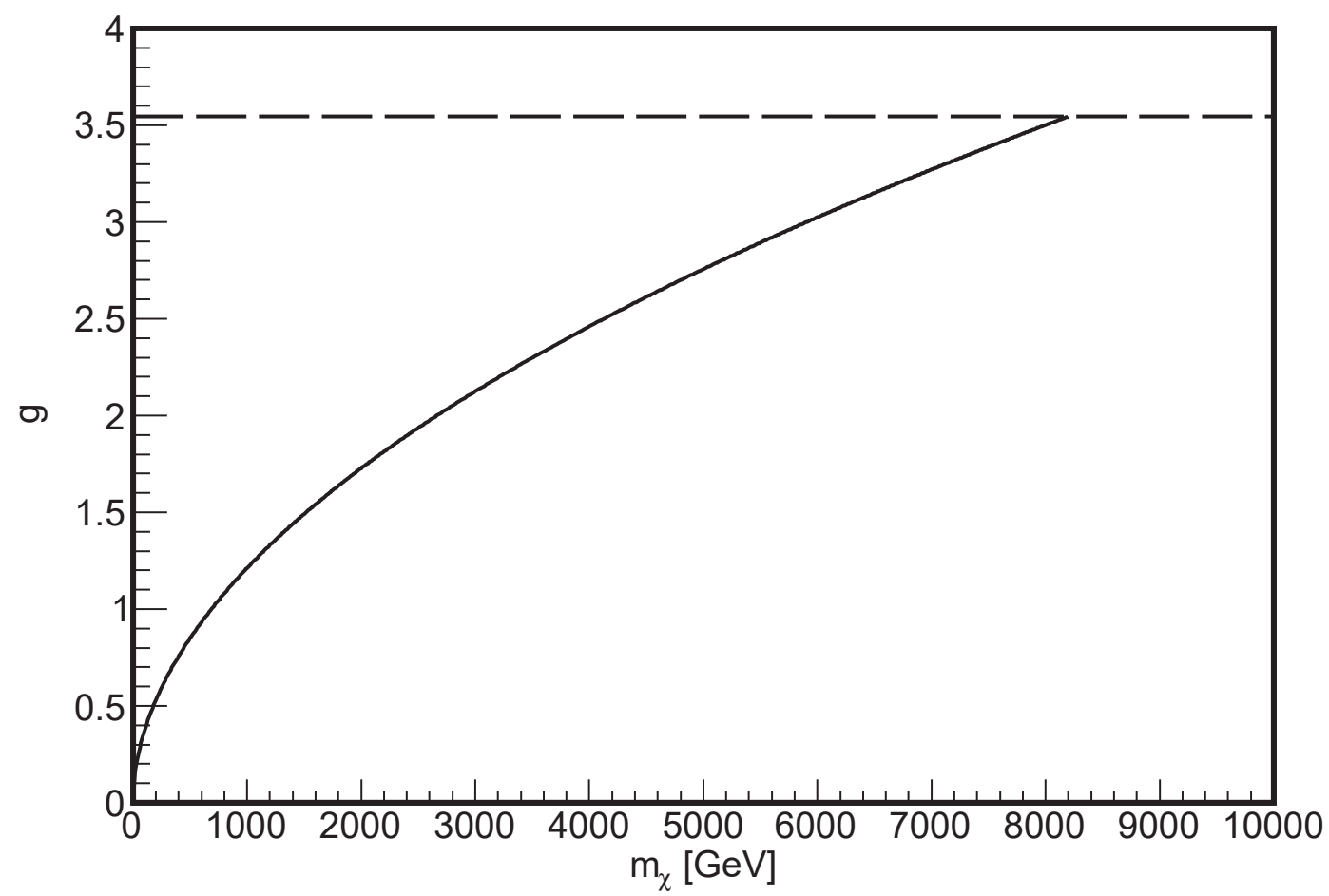

Figure 4.4: A plot of the parameter $g$ from Eq. (4.31) as a function of $m_{\chi}$. The function takes its form because only a particular value of $g$ for a given $m_{\chi}$ results in the correct dark matter relic abundance via thermal freeze-out. A perturbativity limit of $2 \sqrt{\pi}$ is placed on the function as indicated by the horizontal dashed line, which forces $m_{\chi}$ to be less than $8200 \mathrm{GeV}$. 


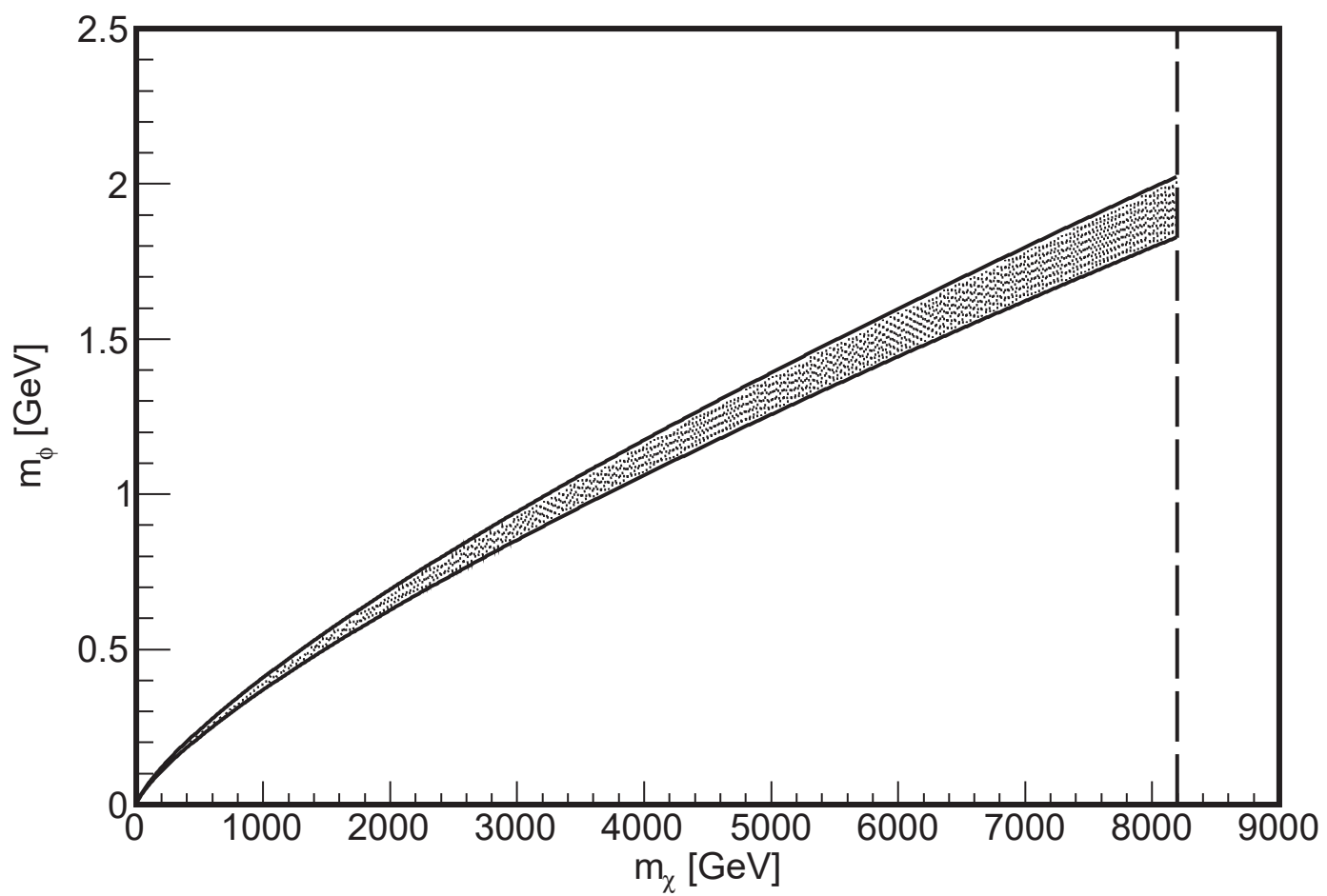

Figure 4.5: A plot comparing the mass of the mediator particle (the $\phi$ ) to the mass of the dark matter particle (the $\chi$ ). The allowed region is shaded and is defined by taking $\sigma_{\text {scatt }} / m_{\chi}$ to lie between 4600 and $6900 \mathrm{GeV}^{-3}$ [2]. The dashed vertical line represents a boundary imposed by Eq. (4.40). This plot turns out to be inaccurate because working in the low-velocity limit in order to simplify $\sigma_{\text {scatt }}$ is a poor approximation when $m_{\phi}$ turns out so very small. See Fig. 4.6 for an accurate portrayal of the relationship between the masses.

At last, it is now time to eliminate $g$ from Eq. (3.36). To wit, combining Eq. (4.31) with Eq. (3.36) yields the following relationship:

$$
m_{\phi}=\left[\frac{8 \alpha m_{\chi}{ }^{3}}{\left(\frac{\sigma_{\text {scatt }}}{m_{\chi}}\right)}\right]^{\frac{1}{4}} .
$$

A plot of Eq. (4.41) with the perturbativity limit on $g$ imposed by Eq. (4.40) is given in Fig. 4.5.

Unfortunately, Fig. 4.5 demonstrates not only that the $\phi$ is light in com- 
parison with the $\chi$, something that was already known, but that the $\phi$ is, in fact, too light. Specifically, it was laid out in Sec. 3.3 that the low-velocity limit of the scattering amplitudes could only be used if the $\chi_{\mathrm{s}}$ were non-relativistic and if $m_{\phi}$ were large compared to the $\chi$ momenta. The first of these conditions is true, but the second most definitely is not. (The issue of velocity dependence in dark matter self-interactions has been discussed in Refs. [35] and $[36]$.

In the galaxy cluster Abell 3827, the relative velocity between colliding dark matter particles is estimated at approximately $1500 \mathrm{~km} / \mathrm{s} \mathrm{[2],} \mathrm{or,} \mathrm{in}$ natural units, $v_{\text {rel }} \approx 0.005$. The typical momentum of an interacting $\chi$ particle in the cluster is therefore

$$
\begin{aligned}
\left|\mathbf{p}_{\chi}\right| & =\frac{1}{2} \gamma m_{\chi} v_{\text {rel }} \\
& =\frac{v_{\text {rel }}}{2 \sqrt{1-\left(\frac{v_{\text {rel }}}{2}\right)^{2}}} m_{\chi} \\
& \approx \frac{0.005}{2 \sqrt{1-\left(\frac{0.005}{2}\right)^{2}}} m_{\chi} \\
& \approx 0.0025 m_{\chi} .
\end{aligned}
$$

Clearly, if $m_{\chi}$ is allowed to be as high as $8200 \mathrm{GeV}$, as Fig. 4.5 suggests, then $\left|\mathbf{p}_{\chi}\right|$ can be as large as $20 \mathrm{GeV}$. Not only is this comparable to $m_{\phi}$, but it is actually an order of magnitude greater than the apparent $2 \mathrm{GeV}$ limit on $m_{\phi}$. It is therefore crucial that the scattering cross section be reevaluated. 
The amplitudes for the processes $\chi \chi \rightarrow \chi \chi$ and $\bar{\chi} \chi \rightarrow \bar{\chi} \chi$ are given in Eq. (3.24) and Eq. (3.26), respectively. Let the function $f$ be defined such that

$$
f(\cos \theta)=\frac{1}{4} \sum_{\text {spins }}\left|\mathcal{M}_{\chi \chi \rightarrow \chi \chi}\right|^{2}
$$

as given in Eq. (3.24), and let the function $g$ be defined such that

$$
g(\cos \theta)=\frac{1}{4} \sum_{\text {spins }}\left|\mathcal{M}_{\bar{\chi} \chi \rightarrow \bar{\chi} \chi}\right|^{2}
$$

as given in Eq. (3.26). If the energy of each incoming particle is $E=\gamma m_{\chi}$, and if the relative velocity of those particles is $v_{\text {rel }}$, and furthermore if the outgoing momentum of each particle is $\gamma m_{\chi} v_{\text {rel }} / 2$, then applying Eq. (3.9) for the cross section and integrating yields

$$
\sigma_{\chi \chi \rightarrow \chi \chi}=\frac{1}{2 E 2 E v_{\text {rel }}} \frac{\gamma m_{\chi} v_{\text {rel }} / 2}{(2 \pi)^{2} 4(2 E)} \int_{0}^{\pi} \int_{0}^{\pi} f(\cos \theta) \sin \theta d \theta d \phi .
$$

If the parameter $I$ is defined such that

$$
I=\int_{0}^{\pi} f(\cos \theta) \sin \theta d \theta
$$

then Eq. (4.45) reduces to

$$
\sigma_{\chi \chi \rightarrow \chi \chi}=\frac{\gamma}{128 \pi E^{3}} I .
$$


Similarly,

$$
\sigma_{\bar{\chi} \chi \rightarrow \bar{\chi} \chi}=\frac{1}{2 E 2 E v_{\mathrm{rel}}} \frac{\gamma m_{\chi} v_{\mathrm{rel}} / 2}{(2 \pi)^{2} 4(2 E)} \int_{0}^{2 \pi} \int_{0}^{\pi} g(\cos \theta) \sin \theta d \theta d \phi
$$

and if the parameter $J$ is defined such that

$$
J=\int_{0}^{\pi} g(\cos \theta) \sin \theta d \theta
$$

then Eq. (4.48) reduces to

$$
\sigma_{\bar{\chi} \chi \rightarrow \bar{\chi} \chi}=\frac{\gamma}{256 \pi E^{3}} J
$$

Following the same logic that lead to Eq. (3.35) in Sec. 3.3, it is apparent that

$$
\sigma_{\text {scatt }}=\frac{\sigma_{\chi \chi \rightarrow \chi \chi}+\sigma_{\bar{\chi} \chi \rightarrow \bar{\chi} \chi}}{2}
$$

meaning the exact scattering cross section with no approximations and not working in any limit is given by

$$
\sigma_{\text {scatt }}=\frac{\gamma m_{\chi}}{256 \pi E^{3}}(I+2 J) .
$$

Thus the equivalent of Eq. (3.36) without approximations is

$$
\frac{\sigma_{\text {scatt }}}{m_{\chi}}=\frac{\gamma}{256 \pi E^{3}}(I+2 J) .
$$


The integrals contained in $I$ and $J$ would be prohibitively time-consuming to perform by hand, and so mathematical software was employed to solve them. The results turn out to be quite long, and are therefore relegated to App. C. Indeed, the final expressions for $I$ and $J$ are so complicated that it is impossible to write $m_{\phi}$ as a function of $m_{\chi}$ as in Eq. (4.41). Rather, a piece of code was written that performs a grid search over the mass region, searching for the $m_{\phi}$ and $m_{\chi}$ values that satisfy Eq. (4.53), where $m_{\phi} / g$ is bounded as described in Eq. (3.37). The output is plotted in Fig. 4.6. Intriguingly, it turns out that the $\phi$ is even lighter than indicated in Fig. 4.5, reaching a maximum possible mass of only about $100 \mathrm{MeV}$. This information will be very useful in the next chapter when determining the $\phi$ 's lifetime through analysis of its Higgs mixing. 


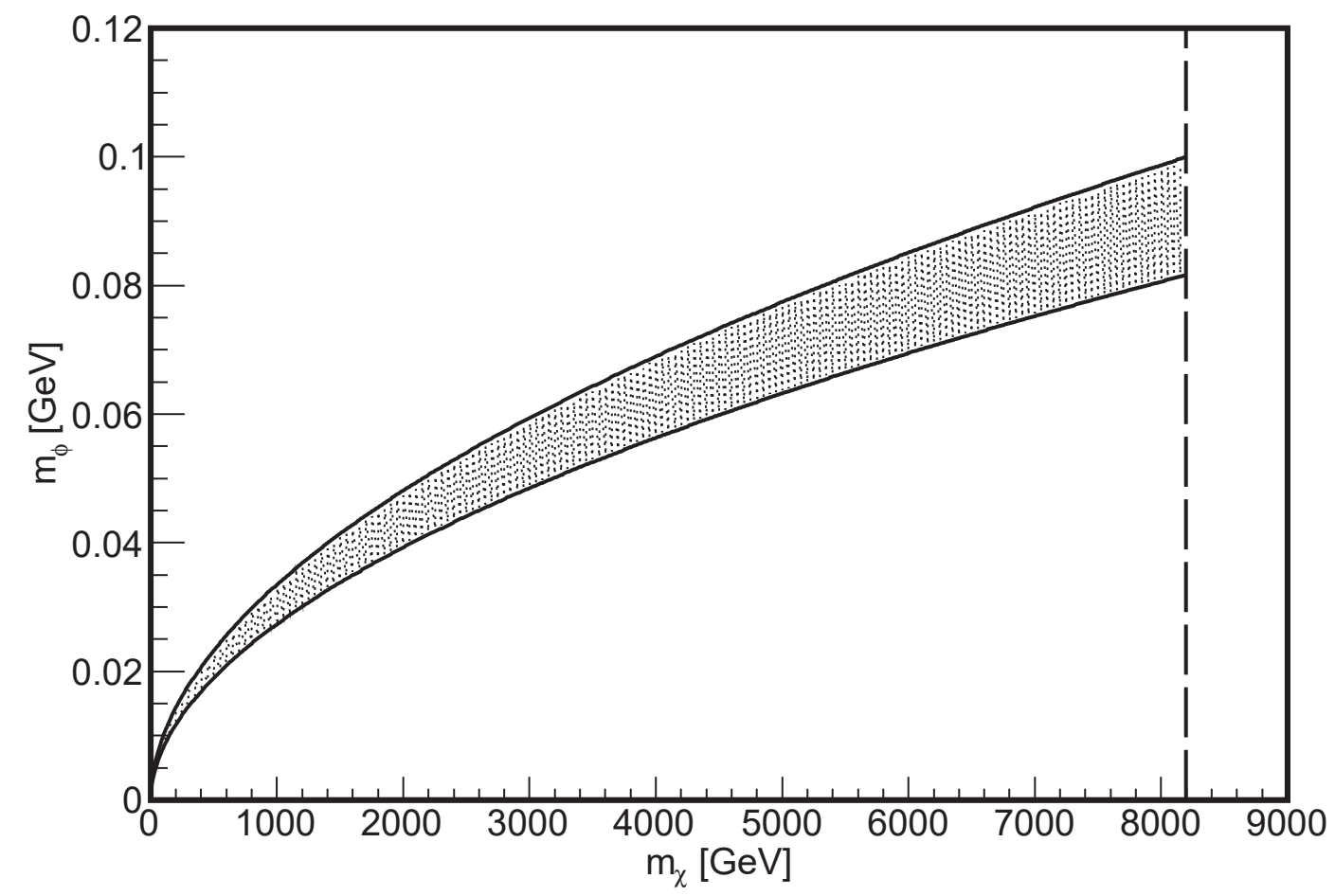

Figure 4.6: A plot comparing the mass of the mediator particle (the $\phi$ ) to the mass of the dark matter particle (the $\chi$ ). The allowed region is shaded and is defined by taking $\sigma_{\text {scatt }} / m_{\chi}$ to lie between 4600 and $6900 \mathrm{GeV}^{-3}$ [2]. The dashed vertical line represents a boundary imposed by Eq. (4.40), which results from a perturbativity constraint on $g$. This plot takes into account the nonzero momentum transfer in the dark matter scattering processes, in contrast with the plot in Fig. 4.5, which does not. 


\section{Chapter 5}

\section{The Dark Sector Meets the Standard Model}

Until now, the dark sector has been dealt with in isolation. It was stated in Ch.

3 that the mediator mixes with the Higgs, and that connection provided the justification for keeping the dark sector in thermal equilibrium with the universal heat bath prior to freeze-out. The mixing relationship was not thoroughly explored, however. Here, the mixing is investigated in detail, and justification is provided for leaving it unaddressed in previous chapters. Furthermore, the full extent of the mixing, taken together with the allowed range of mediator masses, allows for the prediction of mediator lifetimes. This prediction, in turn, provides a lower mass limit for the mediator, which subsequently may be used to set a lower limit on the dark matter fermion mass. 


\subsection{Mixing the $\phi$ with the Higgs}

The general Higgs field, $H$, was originally conceived as a mechanism by which various particles within the Standard Model could acquire mass [37]. Specifically, the requirement for local gauge invariance of the Lagrangian, in combination with spontaneous symmetry breaking in the Higgs field, leads to a vacuum expectation value, $v=246 \mathrm{GeV}$ [38], which is the same at all points in space and is ultimately responsible for giving leptons, quarks and the $W$

and $Z$ bosons their masses. The field is necessarily a doublet and may be written in unitary gauge to reveal the physical Higgs boson, $h$ :

$$
H=\left(\begin{array}{c}
0 \\
\frac{h+v}{\sqrt{2}}
\end{array}\right) .
$$

The Higgs particle is an excitation in the Higgs field and has a mass of $125 \mathrm{GeV}$ [38]. Since the Higgs is a spin-0 boson [37], it should be able to mix with the $\phi$, meaning that, according to our model, the observed Higgs boson is actually part Standard Model Higgs and part $\phi$.

Given the mixing in the model, the Potential of the Lagrangian, $V$, must contain the following terms:

$$
V \supset \mu_{3} H^{\dagger} H \phi+\mu_{4} H^{\dagger} H \phi \phi
$$

where $\mu_{3}$ and $\mu_{4}$ are coupling constants. For simplicity, in particular because 
introducing additional decay modes for the Higgs is undesirable, the second of the terms in Eq. (5.2) is ignored. Thus

$$
V \supset \mu_{3} H^{\dagger} H \phi,
$$

which, after expanding, gives

$$
V \supset \frac{1}{2} \mu_{3} h h \phi+\mu_{3} v_{h} \phi+\frac{1}{2} \mu_{3} v^{2} \phi .
$$

Terms that are quadratic in fields are mass terms [25]. The potential should already include the terms $m_{h}{ }^{2} h h / 2$ and $m_{\phi}{ }^{2} \phi \phi / 2$, the Higgs and mediator mass terms. Consequently, it may be written that

$$
V \supset \frac{1}{2} m_{h}{ }^{2} h h+\mu_{3} v h \phi+\frac{1}{2} m_{\phi}^{2} \phi \phi .
$$

In matrix notation, this becomes

$$
V \supset \frac{1}{2}\left(\begin{array}{ll}
h & \phi
\end{array}\right)\left(\begin{array}{cc}
m_{h}^{2} & \mu_{3} v \\
\mu_{3} v & m_{\phi}^{2}
\end{array}\right)\left(\begin{array}{l}
h \\
\phi
\end{array}\right) .
$$

The $2 \times 2$ matrix in Eq. (5.6) is the mass-squared matrix, $M^{2}$. The fact that $M^{2}$ is not diagonal demonstrates that $h$ and $\phi$ are not mass eigenstates. However, 
there should be a diagonal matrix, $M_{\text {diag }}{ }^{2}$, such that

$$
M_{\mathrm{diag}}^{2}=\left(\begin{array}{cc}
m_{\tilde{h}}^{2} & 0 \\
0 & m_{\tilde{\phi}}^{2}
\end{array}\right) \text {, }
$$

where $m_{\tilde{h}}$ and $m_{\tilde{\phi}}$ are the masses associated with $\tilde{h}$ and $\tilde{\phi}$, the actual mass eigenstates in the mixed system.

An appropriate rotation will diagonalize the mass-squared matrix. Let the rotation matrix $R$ be defined as follows:

$$
R=\left(\begin{array}{cc}
\cos \theta & -\sin \theta \\
\sin \theta & \cos \theta
\end{array}\right)
$$

where $\theta$ is the rotation angle. Then it is possible to map $h$ and $\phi$ onto $\tilde{h}$ and $\tilde{\phi}$ by applying $R$ :

$$
\left(\begin{array}{l}
\tilde{h} \\
\tilde{\phi}
\end{array}\right)=\left(\begin{array}{cc}
\cos \theta & -\sin \theta \\
\sin \theta & \cos \theta
\end{array}\right)\left(\begin{array}{l}
h \\
\phi
\end{array}\right) .
$$

It is easy to show that $R$ is unitary, implying that if $R^{\mathrm{T}}$ is the transpose of $R$, then $R^{\mathrm{T}} R=1$. Therefore, it follows from Eq. (5.9) that

$$
\left(\begin{array}{l}
h \\
\phi
\end{array}\right)=\left(\begin{array}{cc}
\cos \theta & \sin \theta \\
-\sin \theta & \cos \theta
\end{array}\right)\left(\begin{array}{l}
\tilde{h} \\
\tilde{\phi}
\end{array}\right) .
$$


Performing matrix multiplication leads to

$$
h=\tilde{h} \cos \theta+\tilde{\phi} \sin \theta
$$

and

$$
\phi=-\tilde{h} \sin \theta+\tilde{\phi} \cos \theta
$$

The role of $\theta$ is now apparent. If $\theta$ is zero, then the Standard Model Higgs is left alone, and the dark sector remains detached from regular matter; but by allowing $\theta$ to increase, it is possible to introduce more and more mixing until, if $\theta=\pi / 2$, the Standard Model Higgs and the $\tilde{\phi}$ swap places. The angle $\theta$ may therefore be referred to as the dark sector mixing angle.

Now that the true mass eigenstate of the dark matter mediator has been identified, it is the $\tilde{\phi}$, rather than the $\phi$, that will be discussed. This change introduces something of a problem, however. It was stated in Ch. 1 that the mixing of the Higgs and the mediator could be largely ignored, but now the effect of disregarding the mixing is apparent. Recall the interaction Lagrangian from Eq. (3.2):

$$
\mathcal{L}_{\mathrm{DM} \text { int }}=-g \bar{\chi} \chi \phi-\frac{1}{3 !} \tilde{g} \phi \phi \phi
$$

In the end, the second term was ignored, but just keeping the first term and 
substituting in Eq. (5.12) reveals that

$$
\mathcal{L}_{\mathrm{DM} \text { int }}=g \bar{\chi} \chi \tilde{h} \sin \theta-g \bar{\chi} \chi \tilde{\phi} \cos \theta
$$

So long as $\theta$ is sufficiently low, the second term of Eq. (5.14) behaves much as the first term of Eq. (5.13). However, a higher $\theta$ really brings the first term of Eq. (5.14) to the fore, so it is important to identify the maximum possible value for $\theta$.

As it turns out, experimental measurements performed on data generated by the LHC reveal that the Higgs seems to be fairly consistent with Standard Model predictions. The expected number of Higgs decays, $N_{\text {exp }}$, in a particular Higgs production experiment is proportional to the Higgs production cross section multiplied by the appropriate branching ratio. In our model, it is clear from Eq. (5.11) that the Higgs production cross section should in turn be proportional to $\cos ^{2} \theta$ and that the branching ratios are unchanged from their Standard Model values. Thus a measure of the ratio $N_{\text {exp }} / N_{\text {obs }}$, where $N_{\text {obs }}$ is the observed number of Higgs decays, gives an estimate of $\cos ^{2} \theta$. Reference [38] states the aforementioned ratio is $1.09 \pm 0.07$ (stat) \pm 0.08 (syst). Adding the errors in quadrature gives an estimate for the overall error, and this error may be subtracted twice from 1.09 to give a reasonable lower bound of 0.88 . 
It follows that $\cos ^{2} \theta>0.88$, and therefore

$$
\sin ^{2} \theta<0.12 \text {. }
$$

The limit enforced by Eq. (5.15) is not terribly small, so building a stronger case for ignoring the mixing in previous chapters seems advisable. There are three derivations that should be investigated: the scattering cross section, the function for $g$ obtained from thermal freeze-out, and the theoretical upper bound on $g$ imposed by perturbativity requirements.

Consider first the scattering cross section, $\sigma_{\text {scatt }}$, as in Eq. (3.35). Introducing mixing would give an equation of the form

$$
\sigma_{\text {scatt }}=\frac{3 m_{\chi}^{2} g^{4}}{16 \pi}\left(\frac{\cos ^{2} \theta}{m_{\tilde{\phi}^{2}}^{2}}+\frac{\sin ^{2} \theta}{m_{\tilde{h}^{2}}^{2}}\right)^{2} .
$$

Clearly, it makes sense to neglect the second term in parentheses of Eq. (5.16) because $m_{\tilde{h}} \gg m_{\tilde{\phi}}$. Now, to be fair, the approximation that led to Eq. (3.35) was found to be faulty due to the extremely small size of $m_{\phi}$, but an argument similar to this one may be extended to the full scattering cross section in Eq. $(4.52)$.

Next consider $g$ as derived from Eq. (4.19). In general, the annihilation reaction could take the form $\bar{\chi} \chi \rightarrow \tilde{\phi} \tilde{\phi}, \bar{\chi} \chi \rightarrow \tilde{\phi} \tilde{h}$ or $\bar{\chi} \chi \rightarrow \tilde{h} \tilde{h}$. In the event that $m_{\chi} \gg m_{\tilde{h}}$, the final state mass of the $\tilde{h}$ can be neglected just like the 
final state mass of the $\tilde{\phi}$, and the equation with mixing simply becomes

$$
\begin{aligned}
\alpha & =\frac{3 g^{4}}{128 \pi m_{\chi}^{2}}\left(\cos ^{4} \theta+2 \cos ^{2} \theta \sin ^{2} \theta+\sin ^{4} \theta\right) \\
& =\frac{3 g^{4}}{128 \pi m_{\chi}^{2}}\left(\cos ^{2} \theta+\sin ^{2} \theta\right)^{2} \\
& =\frac{3 g^{4}}{128 \pi m_{\chi}^{2}},
\end{aligned}
$$

which is just Eq. (4.19) again. Now, if $m_{\chi} \ll m_{\tilde{h}}$, then

$$
\alpha=\frac{3 g^{4} \cos ^{4} \theta}{128 \pi m_{\chi}^{2}},
$$

but this is only for a relatively small mass range, and even then, $\cos \theta \gtrsim 0.9$, so $g$ can only be about $10 \%$ higher than originally indicated.

Finally, there is the issue of the theoretical upper limit imposed on $g$ by perturbativity. Given that $g<2 \sqrt{\pi}$ without mixing, it follows that

$$
g \cos \theta<2 \sqrt{\pi}
$$

with mixing. Again, this represents no more than a $10 \%$ shift in $g$, so everything seems to be in order. If this argument or the previous two arguments remain unconvincing, it will perhaps be helpful to recall that the limits on the observed dark matter self-interaction cross section, given in Eq. (2.4), are themselves very approximate. Any error introduced by ignoring mixing 
to this point is therefore surely overshadowed by the extreme uncertainty in $\sigma_{\text {scatt }} / m_{\chi}$

Having established that ignoring the mixing up to this point should not have introduced any significant errors in previous calculations, all information pertaining to the $\phi$ will now be applied to the $\tilde{\phi}$, including, most importantly, the $100 \mathrm{MeV}$ upper limit on its mass. Likewise, the $\tilde{h}$ will now be recognized as the observed Higgs, rather than the $h$.

\subsection{Lifetimes for the $\tilde{\phi}$}

Now that its mass range has been specified, it is possible to obtain a range of allowed lifetimes for the $\tilde{\phi}$. In Sec. 4.4 the decay width was defined. The average lifetime of a particle (or just the lifetime), $\tau$, is the inverse of its total decay width $[20,25]$. Given that the only way for the $\tilde{\phi}$ to decay is through

mixing with the Higgs, $\tilde{\phi}$ decays are, in essence, Higgs decays. There is one notable difference, however, which is that many decays for the $125 \mathrm{GeV}$ Higgs are kinematically forbidden for the very light $\tilde{\phi}$. Of all the Higgs decays, then, only the decays to electrons and photons need to be evaluated.

Consider the relevant interaction term between the Higgs and electrons $[25,39]:$

$$
\mathcal{L} \supset-\frac{m_{e}}{v} h \bar{e} e
$$

where $m_{e}$ is the mass of the electron and, again, $v=246 \mathrm{GeV}$ is the Higgs 
vacuum expectation value. Then substitution of Eq. (5.11) into Eq. (5.20) reveals that

$$
\mathcal{L} \supset-\frac{m_{e}}{v} \tilde{h} \cos \theta \bar{e} e-\frac{m_{e}}{v} \tilde{\phi} \sin \theta \bar{e} e,
$$

meaning the Feynman rule for the vertex that gives the $\tilde{\phi}$ decay is $-i\left(m_{e} \sin \theta\right) / v$. Fortunately, a calculation of the decay width for a scalar decaying to a pair of spin-half fermions was already performed in Sec. 4.4 when a theoretical upper bound was placed on $g$. The width for that particular decay process is given in Eq. (4.35). The only differences that arise in calculating $\Gamma_{\tilde{\phi} \rightarrow e^{+} e^{-}}$, the decay width of the process $\tilde{\phi} \rightarrow e^{+} e^{-}$, is the different Feynman rule associated with the vertex and the different fermion mass. It follows that replacing $g$ in Eq. (4.35) with $\left(m_{e} \sin \theta\right) / v$ and replacing $m_{\chi}$ with $m_{e}$ gives

$$
\Gamma_{\tilde{\phi} \rightarrow e^{+} e^{-}}=\frac{m_{e}^{2}}{\pi v^{2} m_{\tilde{\phi}}^{2}} \sin ^{2} \theta\left(\frac{1}{4} m_{\tilde{\phi}}^{2}-m_{e}{ }^{2}\right)^{\frac{3}{2}}
$$

where $\phi$ was changed to $\tilde{\phi}$.

The decay to a photon pair is more complicated and may occur through fermion or $W$ loops. Luckily, adding the $\theta$ dependence to an already-existing equation, just as in Eq. (5.22), will work in this case. The equation giving the Higgs decay width to photons is provided in Ref. [39], and the version used 
to find the decay width for $\tilde{\phi} \rightarrow \gamma \gamma$ is

$$
\Gamma_{\tilde{\phi} \rightarrow \gamma \gamma}=\frac{\alpha_{\mathrm{F}}{ }^{2} m_{\tilde{\phi}}^{3} \sin ^{2} \theta}{256 \pi^{3} v^{2}}\left|\sum_{i} N_{\mathrm{c} i} q_{i}{ }^{2} F_{i}\right|,
$$

where $i$ represents the $i$ th particle participating in a possible decay loop. In order, these particles are each of the six quarks, each of the three charged leptons and the $W$ boson. (Antiparticles do not need to be explicitly counted.) The quantity $N_{\mathrm{c} i}$ is the number of colours associated with the $i$ th particle, 3 for quarks and 1 for everything else, and $q_{i}$ is the charge of the $i$ th particle in units of $e$ (see Table 2). Finally, $F_{i}$ represents two distinct functions, $F_{1 / 2}$ for the fermions and $F_{1}$ for the $W$. The first is defined as [39]

$$
F_{1 / 2}=-2 \nu[1+(1-\nu) f(\nu)]
$$

and the second as

$$
F_{1}=2+3 \nu+3 \nu(2-\nu) f(\nu),
$$

where

$$
f(\nu)= \begin{cases}{\left[\sin ^{-1} \sqrt{\frac{1}{\nu}}\right]^{2}} & \text { for } \nu \geq 1, \\ -\frac{1}{4}\left[\ln \left(\frac{\eta_{+}}{\eta_{-}}\right)-i \pi\right]^{2} & \text { for } \nu<1,\end{cases}
$$

with

$$
\eta_{ \pm}=1 \pm \sqrt{1-\nu}
$$


and

$$
\nu=4 \frac{m_{i}^{2}}{m_{\tilde{\phi}^{2}}^{2}}
$$

Note that $m_{i}$ is the mass of the $i$ th particle and that the $i$ in Eq. (5.26) is not an index, but is instead the imaginary unit. Clearly, performing the calculation to find $\Gamma_{\tilde{\phi} \rightarrow \gamma \gamma}$ by hand, especially for every $m_{\tilde{\phi}}$ value, would be quite a monstrous undertaking, and so a computer program was written to accomplish that task.

With $\Gamma_{\tilde{\phi} \rightarrow e^{+} e^{-}}$and $\Gamma_{\tilde{\phi} \rightarrow \gamma \gamma}$ found, the total decay width, $\Gamma_{\tilde{\phi}}$, is a simple sum of the two, except in the case that the decay to electrons is kinematically forbidden. Thus

$$
\Gamma_{\tilde{\phi}}= \begin{cases}\Gamma_{\tilde{\phi} \rightarrow e^{+} e^{-}}+\Gamma_{\tilde{\phi} \rightarrow \gamma \gamma} & \text { for } m_{\tilde{\phi}} \geq 2 m_{e}, \\ \Gamma_{\tilde{\phi} \rightarrow \gamma \gamma} & \text { for } m_{\tilde{\phi}}<2 m_{e} .\end{cases}
$$

The lifetime is then simply the reciprocal:

$$
\tau=\frac{1}{\Gamma_{\tilde{\phi}}}
$$

Before plotting the allowed region for $\tau$ as a function of $m_{\tilde{\phi}}$, there is one more point worth considering. As described in Ch. 4, the contents of the universe were in thermal equilibrium during its earliest epochs. At some point, however, nucleons were able to combine to give rise to isotopes of hydrogen 
and helium, as well as low abundances of heavier elements. This process is called Big Bang nucleosynthesis [4, 40], and it started on the order of seconds after the Big Bang. Crucially, the observed ratios of different isotopes indicate that there could not have been a huge reservoir of $\tilde{\phi}$ s around during this time because their high-energy decay products would have destroyed many newlyformed nuclei. Therefore, conservatively speaking, if nucleosynthesis started one second after the Big Bang, then it would make sense to set the maximum lifetime for the $\tilde{\phi}$ a couple of orders of magnitude lower, or about $0.01 \mathrm{~s}$. That way, the $\tilde{\phi}$ s would have had enough time to decay before nuclei started to form. (Note that Big Bang nucleosynthesis happened much later than any of the freeze-out times from Ch. 4, so our model is safe in that regard.)

A plot of the allowed mass-lifetime region is provided in Fig. 5.1. Note the upper bound on the lifetime at $0.01 \mathrm{~s}$ (horizontal dashed line) arising from consideration of Big Bang nucleosynthesis, as well as the upper bound on the mass at $100 \mathrm{MeV}$ (vertical dashed line). The solid curve is taken by setting $\sin ^{2} \theta=0.12$. The overall lifetime range is

$$
3.2 \times 10^{-10} \mathrm{~s}<\tau<10^{-2} \mathrm{~s}
$$

The lower range of this lifetime is many orders of magnitude longer than the Higgs lifetime, which makes sense because of the mass difference between the two particles. Interestingly, the upper-left corner of the plot, where the 


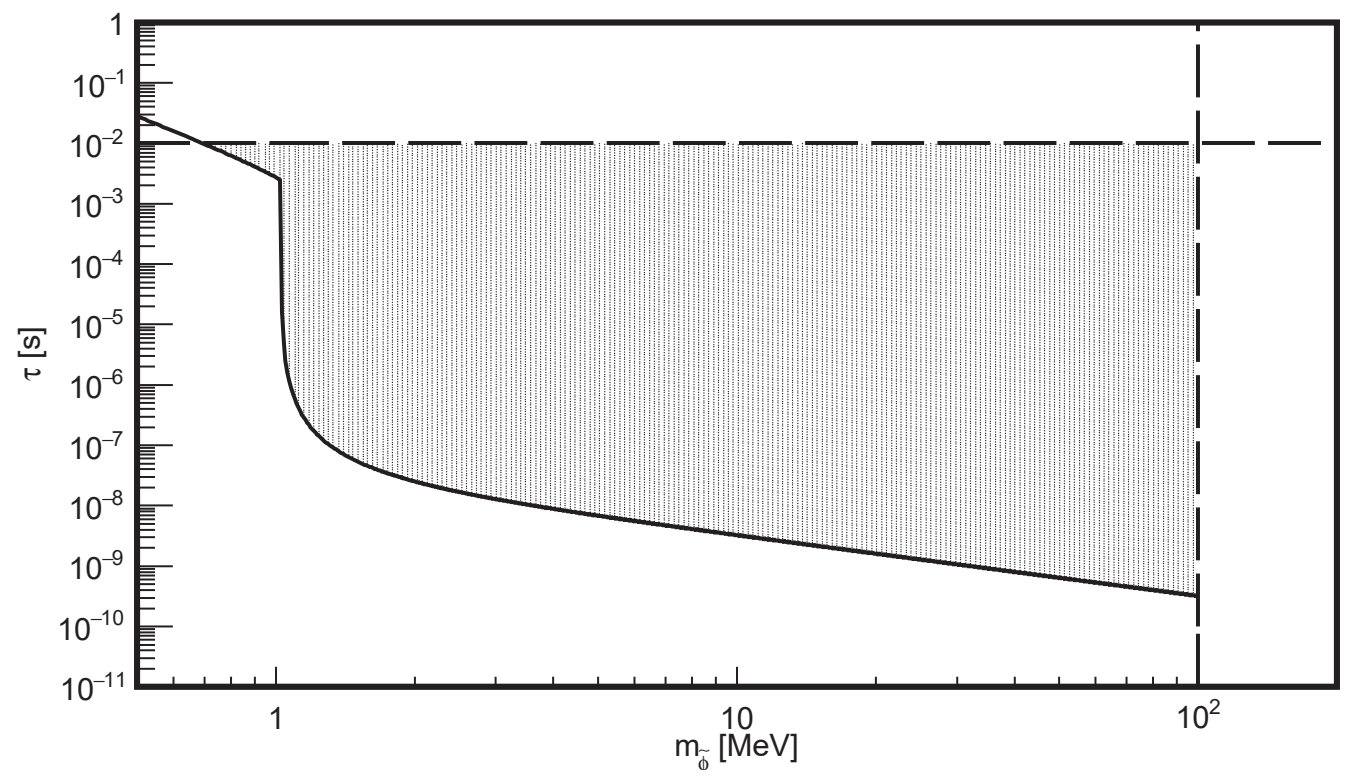

Figure 5.1: A logarithmic plot revealing the allowed lifetimes for the $\tilde{\phi}$. The allowed region is shaded and lies above the solid curve, which depicts the lifetimes if the mixing angle is taken such that $\sin ^{2} \theta=0.12$. The horizontal dashed line at $0.01 \mathrm{~s}$ that bounds from above represents a maximum possible lifetime arising from arguments having to do with Big Bang nucleosynthesis. The vertical dashed line bounds on the right and exists because the $\tilde{\phi}$ cannot have a mass any greater than $100 \mathrm{MeV}$. The sharp turning point at approximately $1 \mathrm{MeV}$ occurs because that is where $\tilde{\phi} \rightarrow e^{+} e^{-}$becomes kinematically permitted.

horizontal dashed line meets the solid curve, reveals a lower limit of $0.69 \mathrm{MeV}$ on $m_{\tilde{\phi}}$. Thus,

$$
0.69 \mathrm{MeV}<m_{\tilde{\phi}}<100 \mathrm{MeV}
$$

This lower limit for $m_{\tilde{\phi}}$ can be used together with Fig. 4.6 to place a lower limit on $m_{\chi}$, revealing that

$$
0.35 \mathrm{GeV}<m_{\chi}<8200 \mathrm{GeV}
$$

From an experimentalist's perspective, it would be nice to know the min- 
imum distance, $d_{\min }$, a $\tilde{\phi}$ might travel before decaying. That way it might be possible to detect a $\tilde{\phi}$ in a Higgs production experiment by locating outgoing particles from a displaced vertex. If the $\tilde{\phi}$ is $100 \mathrm{MeV}, \Gamma_{\tilde{\phi} \rightarrow \gamma \gamma}$ is only $0.02 \%$ of the total decay width, so the decay would almost certainly produce an electron-positron pair at that mass. The minimum lifetime for the $\tilde{\phi}$ is $\tau_{\min }=3.2 \times 10^{-10} \mathrm{~s}$, so the minimum possible travel distance is

$$
\begin{aligned}
d_{\text {min }} & =c \tau_{\text {min }} \\
& =\left(2.998 \times 10^{10} \mathrm{~cm} / \mathrm{s}\right)\left(3.2 \times 10^{-10} \mathrm{~s}\right) \\
& =9.6 \mathrm{~cm},
\end{aligned}
$$

where the speed of light is used for the velocity because the low-mass $\tilde{\phi}$ would have to be highly relativistic. 


\section{Chapter 6}

\section{Conclusions}

This thesis was an attempt to explain the observed offset of galaxies and their dark matter halos in Ref. [1] by constructing a simple dark matter model made up of the fermion $\chi$ and its antiparticle $\bar{\chi}$, as well as the scalar mediator $\tilde{\phi}$ (introduced to "replace" the $\phi$ after mixing effects were taken into account). It was shown that the observational constraints on the scattering cross section demand an extremely light mediator in comparison with the fermion mass, and it was also shown that the fermion mass scales as the fourth power of the mediator mass. Moreover, a large range of lifetimes appear to be acceptable

for the $\tilde{\phi}$, from $3.2 \times 10^{-10} \mathrm{~s}$ to $10^{-2} \mathrm{~s}$; but this range is related to $\theta$, the dark sector mixing angle, and could be restricted by more accurate data on Higgs decays. Nevertheless, as it stands, it seems that detection of a $\tilde{\phi}$ might be possible given its minimum possible decay length of $9.6 \mathrm{~cm}$.

Two important constraints were imposed on this model. The first was a 
theoretical upper bound on $g$, the mediator-fermion coupling strength, showing that $g<2 \sqrt{\pi}$. The second was the maximum allowed lifetime for the $\tilde{\phi}$, which arose from consideration of Big Bang nucleosynthesis. These two constraints imposed upper and lower limits on the particle masses, revealing that the $\tilde{\phi}$ has a mass falling somewhere between $0.69 \mathrm{MeV}$ and $100 \mathrm{MeV}$, while the $\chi$ has a mass between $0.35 \mathrm{GeV}$ and $8200 \mathrm{GeV}$.

The overarching goal of this thesis was not necessarily to make the claim that our model is definitively the reason behind the anomalous observations of Abell 3827, though the allowed parameter space suggests such a thing is possible. Rather, it was to use a simple model to illustrate what sort of masses and lifetimes one might expect for a particular type of self-interacting dark matter. Hopefully, these findings might be extended, at least qualitatively, to inform other more complex theories; for, should additional evidence turn up in support of self-interacting dark matter, this area of research will no doubt receive a great deal of attention. 


\section{Appendix A}

\section{Units}

Natural units are a way of expressing various quantities in terms of energy and are commonly used in particle physics and cosmology [20]. They are defined by taking $\hbar=c=k_{\mathrm{B}}=1$.

Natural units tend to make calculations simpler, in particular because they allow physicists to leave out factors of $\hbar, c$ and $k_{\mathrm{B}}$ in their calculations. Furthermore, working in natural units allows a more meaningful and immediate comparison of, say, the momentum of one particle and the mass of another because the units are the same in both cases. Unfortunately, natural units come with some drawbacks. Specifically, it can be difficult to switch between natural units and SI units, especially if a quantity expressed in natural units needs to be converted to SI units when the dimensionality in SI units is unknown. This set of circumstances would require correct placement of $\hbar, c$ and $k_{\mathrm{B}}$ factors, which would be difficult if dimensionality is not carefully considered during 
the derivation of the quantity in question.

To help with conversions from SI to natural units, some of the basic units of the SI system - the kilogram $(\mathrm{kg})$, the metre $(\mathrm{m})$, the second $(\mathrm{s})$ and the Kelvin (K) - are here expressed in natural units. Let the subscript SI denote a constant in SI units and the subscript nat denote a constant in natural units.

Then

$$
\begin{aligned}
1 \mathrm{~kg} & =1 \mathrm{~kg} \times\left(\frac{c_{\text {SI }}}{c_{\text {nat }}}\right)^{2} \\
& =1 \mathrm{~kg} \times\left(\frac{2.998 \times 10^{8} \mathrm{~m} \mathrm{~s}^{-1}}{1}\right)^{2} \\
& =8.988 \times 10^{16} \mathrm{~J} \\
& =8.988 \times 10^{16} \mathrm{~J} \times \frac{1 \mathrm{eV}}{1.602 \times 10^{-19} \mathrm{~J}} \\
& =5.610 \times 10^{35} \mathrm{eV} .
\end{aligned}
$$

Similarly,

$$
\begin{aligned}
& 1 \mathrm{~m}=1 \mathrm{~m} \times \frac{\hbar_{\mathrm{nat}} c_{\mathrm{nat}}}{\hbar_{\mathrm{SI}} c_{\mathrm{SI}}}=5.065 \times 10^{6} \mathrm{eV}^{-1}, \\
& 1 \mathrm{~s}=1 \mathrm{~s} \times \frac{\hbar_{\text {nat }}}{\hbar_{\mathrm{SI}}}=1.518 \times 10^{15} \mathrm{eV}^{-1}
\end{aligned}
$$

and

$$
1 \mathrm{~K}=1 \mathrm{~K} \times \frac{k_{\mathrm{B} \mathrm{SI}}}{k_{\mathrm{B} \text { nat }}}=8.602 \times 10^{-5} \mathrm{eV}
$$

Results are summarized in Table A.1. 
Table A.1 - Unit Conversions

\begin{tabular}{lr}
\hline \hline SI Unit & In Natural Units \\
\hline $1 \mathrm{~kg}$ & $5.610 \times 10^{35} \mathrm{eV}$ \\
$1 \mathrm{~m}$ & $5.065 \times 10^{6} \mathrm{eV}^{-1}$ \\
$1 \mathrm{~s}$ & $1.518 \times 10^{15} \mathrm{eV}^{-1}$ \\
$1 \mathrm{~K}$ & $8.602 \times 10^{-5} \mathrm{eV}$ \\
\hline
\end{tabular}

Table A.1: Some of the basic units of the SI system converted to their equivalent values in natural units. 


\section{Appendix B}

\section{Application of the Runge-Kutta Method to Freeze-Out}

Suppose an ordinary, first-order differential equation given by

$$
\frac{d y}{d x}=f(x, y)
$$

describes some phenomenon of interest, where $y$ is the dependent variable and $x$ is the independent variable. Furthermore, let $y$ be known for a single point $x_{0}$, and call it $y_{0}$. If the function $y(x)$ is unknown, then a Taylor series expansion about $x_{0}$ should give $y(x)$.

Let $\Delta x$ be some small displacement along the $x$ axis. Then a Taylor series expansion gives

$$
y(x+\Delta x)=y(x)+\frac{d y}{d x}(\Delta x)+\frac{1}{2} \frac{d^{2} y}{d x^{2}}(\Delta x)^{2}+\ldots .
$$


If it were absolutely necessary to include the third term and terms of even higher order, then the Taylor series expansion would not be very helpful. After all, in principle - and often in practice - only Eq. (B.1) is known. Fortunately, thanks to the mean value theorem [41], it is easy to show that there must be a particular $x$ value, $x_{\mathrm{p}}$, falling between $x$ and $x+\Delta x$, such that

$$
y(x+\Delta x)=y(x)+\left.\frac{d y}{d x}\right|_{x=x_{\mathrm{p}}}(\Delta x),
$$

and the higher-order terms may successfully be ignored.

Using Eq. (B.3), it should be possible to begin at $x_{0}$ and to find $y$ by moving along the $x$ axis in small increments of size $\Delta x$, updating $x_{0}$ and $y_{0}$ at each step. Thus the problem becomes finding $x_{\mathrm{p}}$ for each iteration of the process. One of the most popular choices for accomplishing this goal, thanks to its accuracy and computational efficiency, is the fourth-order Runge-Kutta method [41], which calculates $d y / d x$ at a value approximately equal to $x_{\mathrm{p}}$. The method is provided below:

$$
y(x+\Delta x)=\frac{1}{6}\left[f\left(x_{1}, y_{1}\right)+2 f\left(x_{2}, y_{2}\right)+2 f\left(x_{3}, y_{4}\right)+f\left(x_{4}, y_{4}\right)\right]
$$


where

$$
\begin{aligned}
& y_{1}=y(x), \\
& y_{2}=y(x)+\frac{1}{2} f\left(x_{1}, y_{1}\right) \Delta x, \\
& y_{3}=y(x)+\frac{1}{2} f\left(x_{2}, y_{2}\right) \Delta x, \\
& y_{4}=y(x)+\frac{1}{2} f\left(x_{3}, y_{3}\right) \Delta x, \\
& x_{1}=x, \\
& x_{2}=x+\frac{1}{2} \Delta x, \\
& x_{3}=x+\frac{1}{2} \Delta x \text { and } \\
& x_{4}=x+\Delta x .
\end{aligned}
$$

The fourth-order Runge-Kutta method was used to solve Eq. (4.28) by way of code written in the $\mathrm{C}$ programming language. In order to obtain the solution, it was discovered that the step size, $\Delta x$, needed to be extraordinarily small. Consequently, it originally took roughly one week to generate a single solution curve for $Y$. That said, there were two tricks employed to speed up the process, resulting in a run time of about a half an hour (for $m_{\chi}$ around $100 \mathrm{GeV})$; and even though a half-hour run time is still quite impractical, these two tricks are worth discussing here.

The first trick is fairly straightforward. It is stated in Ref. [26] that one should begin by assuming that $Y=Y_{\text {eq }}$ when $x$ is approximately equal to 1 . 
(These constitute the $y_{0}$ and $x_{0}$ starting point for the Runge-Kutta method). However, several runs of the program demonstrated that taking $Y=Y_{\text {eq }}$ at $x=10$ and using that as the starting point instead made no detectable impact on the results. This fact is particularly useful in light of what follows.

The second trick is to allow the step size to grow as the solution curve is generated. In particular, if $\Delta x_{\text {new }}$ is the step size at some $x$, and $\Delta x_{0}$ is the original step size, then for p-wave annihilation it is acceptable to take

$$
\Delta x_{\text {new }}=\left(\Delta x_{0}\right) x^{3} .
$$

To explain why a growing step size is allowed, consider the case of $m_{\chi}=$ $100 \mathrm{GeV}$. The differential equation of interest is Eq. (4.28). To reiterate,

$$
\frac{d Y}{d x}=\left(6 \alpha d_{f s} \sqrt{\frac{\pi}{45 G d_{f}}}\right)\left(Y_{\mathrm{eq}}{ }^{2}-Y^{2}\right) \frac{m_{\chi}}{x^{3}},
$$

where the variables and constants are all defined in Ch. 4. The important aspect of Eq. (B.7) to note is the tension between the first and second factors in parentheses. For the correct choice of $\alpha\left(1.41 \times 10^{-8} \mathrm{GeV}^{-2}\right)$, the leading factor is large in comparison with the small difference between $Y_{\text {eq }}{ }^{2}$ and $Y^{2}$, and essentially overshadows it, drowning out the important behaviour within the function. To counteract the largeness of the leading factor, an appropriately small starting step, $\Delta x_{0}$, must be chosen $\left(\Delta x_{0}=5 \times 10^{-12}\right)$. For illustrative 
purposes, note the effect of increasing $m_{\chi}$ : this is essentially akin to raising the leading factor, and so the run time must increase linearly with mass in agreement with a required linear drop in step size. The saving grace here is the factor of $x^{3}$ in the denominator. So long as $\Delta x_{0}$ is small enough in the beginning, the same ratio of $x^{3}$ to $\Delta x$ may be preserved by allowing the step size to grow as in Eq. (B.6). 


\section{Appendix $\mathrm{C}$}

\section{The Scattering Cross Section Integrals}

In Sec. 4.4, the quantities $I$ and $J$ were defined such that

$$
I=\int_{0}^{\pi} f(\cos \theta) \sin \theta d \theta
$$

and

$$
J=\int_{0}^{\pi} g(\cos \theta) \sin \theta d \theta
$$

where

$$
f(\cos \theta)=\frac{1}{4} \sum_{\text {spins }}\left|\mathcal{M}_{\chi \chi \rightarrow \chi \chi}\right|^{2}
$$

and

$$
g(\cos \theta)=\frac{1}{4} \sum_{\text {spins }}\left|\mathcal{M}_{\bar{\chi} \chi \rightarrow \bar{\chi} \chi}\right|^{2}
$$


The amplitudes in Eq. (C.3) and Eq. (C.4) are given in Eq. (3.24) and Eq. (3.26), respectively. The worked-out expressions were too long to include within the text of Sec. 4.4, so they are given here:

$$
\begin{aligned}
& I=4 g^{4}\left\{4 ( E - m _ { \chi } ) ( E + m _ { \chi } ) ( 2 E ^ { 2 } + m _ { \phi } { } ^ { 2 } - 2 m _ { \chi } { } ^ { 2 } ) \left(12 E^{2} m_{\phi}{ }^{2}+5 m_{\phi}{ }^{4}\right.\right. \\
& \left.-28 m_{\phi}{ }^{2} m_{\chi}{ }^{2}+32 m_{\chi}{ }^{4}\right)+m_{\phi}{ }^{2}\left(4 E^{2}+m_{\phi}{ }^{2}-4 m_{\chi}{ }^{2}\right)\left[12 E^{2} m_{\phi}{ }^{2}+5 m_{\phi}{ }^{4}\right. \\
& \left.\left.-28 m_{\phi}{ }^{2} m_{\chi}{ }^{2}-16 E^{2}+32 m_{\chi}{ }^{4}\right]\left[2 \ln \left(m_{\phi}\right)-\ln \left(4 E^{2}+m_{\phi}{ }^{2}-4 m_{\chi}{ }^{2}\right)\right]\right\}\left[8 m_{\phi}{ }^{2}(E\right. \\
& \left.\left.-m_{\chi}\right)\left(E+m_{\chi}\right)\left(4 E^{2}+m_{\phi}{ }^{2}-4 m_{\chi}{ }^{2}\right)\left(2 E^{2}+m_{\phi}{ }^{2}-2 m_{\chi}{ }^{2}\right)\right]^{-1}
\end{aligned}
$$

and

$$
\begin{aligned}
& J=4 g^{4}\left\{4 ( E - m _ { \chi } ) ( E + m _ { \chi } ) \left[48 E^{6} m_{\phi}{ }^{2}+22 E^{4} m_{\phi}{ }^{4}-15 E^{2} m_{\phi}{ }^{6}+2 m_{\phi}{ }^{8}\right.\right. \\
& +m_{\phi}{ }^{2} m_{\chi}{ }^{2}\left(-120 E^{4}+44 E^{2} m_{\phi}{ }^{2}-7 m_{\phi}{ }^{4}\right)+2 m_{\chi}{ }^{4}\left(32 E^{4}-8 E^{2} m_{\phi}{ }^{2}+5 m_{\phi}{ }^{4}\right) \\
& \left.-8 m_{\phi}{ }^{2} m_{\chi}{ }^{6}\right]+m_{\phi}{ }^{2}\left(4 E^{2}-m_{\phi}{ }^{2}\right)\left(2 E^{2}+m_{\phi}{ }^{2}-2 m_{\chi}{ }^{2}\right)\left[2 \left(-4 m_{\phi}{ }^{4}+9 m_{\phi}{ }^{2} m_{\chi}{ }^{2}\right.\right. \\
& \left.-4 m_{\chi}{ }^{4}+17 E^{2} m_{\phi}{ }^{2}-28 E^{2} m_{\chi}{ }^{2}\right) \ln \left(m_{\phi}\right)-\left(m_{\phi}{ }^{2} m_{\chi}{ }^{2}-4 m_{\chi}{ }^{4}+E^{2} m_{\phi}{ }^{2}\right. \\
& \left.+4 E^{2} m_{\chi}{ }^{2}\right) \ln \left(4 E^{2}+m_{\phi}{ }^{2}-4 m_{\chi}{ }^{2}\right)+4\left(-4 E^{2}+m_{\phi}{ }^{2}\right)\left(m_{\phi}{ }^{2}-2 m_{\chi}{ }^{2}\right) \ln \left(2 E^{2}\right. \\
& \left.\left.\left.+m_{\phi}{ }^{2}-2 m_{\chi}{ }^{2}\right)\right]\right\}\left[2 ( - 4 E ^ { 2 } m _ { \phi } + m _ { \phi } { } ^ { 3 } ) ^ { 2 } ( E - m _ { \chi } ) ( E + m _ { \chi } ) \left(2 E^{2}+m_{\phi}{ }^{2}\right.\right. \\
& \left.\left.-2 m_{\chi}{ }^{2}\right)\right]^{-1}
\end{aligned}
$$

where $E$ is the energy of a $\chi$ (or $\bar{\chi}$ ) in the centre-of-mass frame, $m_{\chi}$ is the mass of a $\chi($ or $\bar{\chi}), m_{\phi}$ is the $\phi$ mass and $g$ is the mediator-fermion coupling. 


\section{Bibliography}

[1] R. Massey et al., "The behaviour of dark matter associated with 4 bright cluster galaxies in the $10 \mathrm{kpc}$ core of Abell 3827," Mon. Not. Roy. Astron. Soc., vol. 449, p. 3393, 2015. arXiv:1504.03388 [astro-ph.CO].

[2] F. Kahlhoefer, K. Schmidt-Hoberg, J. Kummer, and S. Sarkar, "On the interpretation of dark matter self-interactions in Abell 3827," Mon. Not. Roy. Astron. Soc., vol. 452, pp. L54-L58, 2015. arXiv:1504.06576 [astroph.CO].

[3] C. Patrignani et al., "The review of particle physics," Chin. Phys. C, vol. 40, p. 100001, 2016. Particle Data Group, wwwpdg.lbl.gov/index.html.

[4] S. Dodelson, Modern Cosmology. United States of America: Academic Press, 2003.

[5] G. B. Gelmini, "TASI 2014 lectures: The hunt for dark matter," 2015. arXiv:1502.01320.

[6] G. Bertone, "The moment of truth for WIMP dark matter," Nature, vol. 468, pp. 389-393, 2010. arXiv:1011.3532.

[7] S. Chang et al., "Effective WIMPs," Phys. Rev. D, vol. 89, p. 15011, 2014. arXiv:1307.8120.

[8] R. Campbell et al., "Implications for the observation of dark matter self-interactions for singlet scalar dark matter," Phys. Rev. D, vol. 92, p. 55031, 2015. arXiv:1509.01793.

[9] J. M. Cline et al., "Multimediator models for the galactic center gamma ray excess," Phys. Rev. D, vol. 91, p. 115010, 2015.

[10] U. J. L. Verrier, Recherches sur les mouvements de la planète Herschel. Bachelier, Imprimeur-libraire, 1846. 
[11] F. Zwicky, "Republication of: The redshift of extragalactic nebulae," General Relativity and Gravitation, vol. 41, pp. 207-224, 2009.

[12] K. W. Whitten, R. E. Davis, M. L. Peck, and G. G. Stanley, Chemistry Eighth Edition. Belmont, California: Brooks/Cole Cengage Learning, 2007.

[13] F. Watson and S. Gary, "How do astronomers use light to study stars and planets?." http://www.abc.net.au/science/articles/2010/10/07/3012690.htm, 2010.

[14] J. W. Rohlf, Modern Physics from $\alpha$ to $Z^{0}$. United States of America: John Wiley and Sons, Inc., 1994.

[15] E. Kolb and M. Turner, The Early Universe. Redwood City, California: Addison Wesley, 1990.

[16] D. Cline, "The search for dark matter," Scientific American, vol. 3, pp. 50-59, 2003.

[17] C. Palma, "The rotation curve of the Milky Way." http://www.eeducation.psu.edu/astro801/content/18_p8.html, 2014.

[18] A. Einstein, "Lens-like action of a star by the deviation of light in the gravitational field," Science, vol. 84, pp. 506-507, 1936.

[19] R. Nemiroff and J. Bonnell, "Astronomy picture of the day." http://www.apod.nasa.gov/apod/ap950710.html, 1995.

[20] D. Griffiths, Introduction to Elementary Particles. Weinheim, Germany: Wiley-VCH, 2008.

[21] N. S. Israel and J. W. Moffat, "The train wreck cluster and bullet cluster explained by modified gravity without dark matter," 2016. arXiv:1606.09128 [astro-ph.CO].

[22] M. S. Turner, "Cosmological parameters," 1999. arXiv:astro-ph/9904051.

[23] J. Alexander et al., "Dark sectors 2016: Community workshop," 2016. arXiv:1608.08632.

[24] M. Markevitch et al., "Direct constraints on the dark matter selfinteraction cross-section from the merging galaxy cluster 1e 0657-56," Astrophys J, vol. 606, pp. 819-824, 2004. arXiv:astro-ph/0309303v2.

[25] M. Peskin and D. Schroeder, An Introduction to Quantum Field Theory. Reading, Massachusetts: Addison-Wesley Publishing Company, 1995. 
[26] G. Steigman, B. Dasgupta, and J. Beacom, "Precise relic WIMP abundance and its impact on searches for dark matter annihilation," Phys. Rev. D, vol. 86, p. 23506, 2012. arXiv:1204.3622 [hep-ph].

[27] G. Bonner, Topics in Higgs Physics and Dark Matter. Ottawa, Canada: Carleton University, Physics Dept., 2016.

[28] R. Baierlein, Thermal Physics. Cambridge, United Kingdom: Cambridge University Press, 1999.

[29] G. Cowan, Statistical Data Analysis. Oxford, United Kingdom: Oxford University Press, 1989.

[30] D. Griffiths, Introduction to Quantum Mechanics Second Edition. Upper Saddle River, New Jersey: Pearson Prentice Hall, 2005.

[31] M. Cannoni, "Exact theory of freeze out," Eur. Phys. J. C, vol. 75, p. 106, 2015. arXiv:1407.4108.

[32] T. S. Coleman and M. Roos, "Effective degrees of freedom during the radiation era," Phys. Rev. D, vol. 68, 2003. arXiv:astro-ph/0304281.

[33] C. A. Egan and C. H. Lineweaver, "A larger estimate of the entropy of the universe," Astrophys. J, vol. 710, pp. 1825-1834, 2010. arXiv:0909.3983.

[34] V. Barger, J. Hewett, and R. Phillips, "New constraints on the charged higgs sector in two-higgs-doublet models," Phys. Rev. D, vol. 41, pp. 3421-3441, 1990.

[35] A. Loeb and N. Weiner, "Cores in dwarf galaxies from dark matter with a Yukawa potential," Phys. Rev. Lett., vol. 106, p. 171302, 2011. arXiv:1011.6374.

[36] S. Tulin, H. Yu, and K. M. Zurek, "Beyond collisionless dark matter: Particle physics dynamics for dark matter halo structure," 2013. arXiv:1302.3898.

[37] C. Burgess and G. Moore, The Standard Model: A Primer. Cambridge, United Kingdom: Cambridge University Press, 2007.

[38] ATLAS and CMS, "Measurements of the Higgs boson production and decay rates and constraints on its couplings from a combined ATLAS and CMS analysis of the LHC $p p$ collision data at $\sqrt{s}=7$ and $8 \mathrm{TeV}$," JHEP, vol. 8, pp. 1-104, 45. arXiv:1606.02266.

[39] J. F. Gunion, H. E. Haber, G. Kane, and S. Dawson, The Higgs Hunter's Guide. United States of America: Addison-Wesley Publishing Company, 1990. 
[40] K. Jedamzik and M. Pospelov, "Big Bang nucleosynthesis and particle dark matter," arXiv:0906.2087.

[41] N. J. Giordano and H. Nakanishi, Computational Physics Second Edition. United States of America: Pearson Prentice Hall, 2006. 